\title{
9. PALEOGENE AND NEOGENE BENTHIC FORAMINIFERS FROM ROCKALL PLATEAU ${ }^{1}$
}

\author{
John W. Murray, Department of Geology, University of Exeter ${ }^{2}$ \\ With a contribution by J. F. Weston
}

\begin{abstract}
Sites 552 to 555 were drilled on a sediment-starved subsiding passive margin. The Cenozoic sedimentary successions have yielded benthic foraminiferal assemblages of late Paleocene to Pleistocene age. Paleoecological analysis, using comparisons with data on modern assemblages, shows that the environments ranged from marginal marine in the late Paleocene to early Eocene to bathyal in the Oligocene and Neogene. Comparison with other DSDP results suggests that faunal communication between the Rockall area and the Norwegian Sea existed from the early Eocene and that cool deep water entered the area in the late Eocene to early Oligocene. The early Miocene bathyal assemblages are clearly recognizable as of North Atlantic Deep Water type.
\end{abstract}

\section{INTRODUCTION}

Four sites (Holes 552, 552A; 553, 553A, 553B; 554, 554A; and 555) were drilled on the sediment-starved passive margin to the southwest of Rockall Plateau (Table 1, Fig. 1). Detailed studies of the benthic foraminifers were carried out both on board Glomar Challenger and subsequently in Exeter, to interpret the subsidence and environmental history of the area. Previously, Sites 403 and 404 , drilled on Leg 48 , had shown that this margin had undergone subsidence from around sea-level in the late Paleocene to early Eocene to bathyal depths $(>2000 \mathrm{~m})$ by the Neogene. It was hoped that the use of the hydraulic piston corer on Leg 81 would greatly improve the quality of sampling and thus permit more detailed studies to be carried out. This proved to be the case at Hole 552A. All other sites were drilled using conventional methods but nevertheless a more complete record was obtained than during Leg 48 . Sites 554 , on the outer high, and 555, drilled to the north of Edoras Bank, have added much new information about previously undrilled areas.

An account of downhole occurrence of the benthic foraminiferal assemblages is given in each of the site chapters and is not repeated here. The primary data are listed in Tables 5 to 11. In this chapter comparisons are made between the sites, to draw out the similarities and differences, to describe the faunas in a stratigraphic sequence, to interpret the conditions of deposition, and to discuss the implications of these results for the understanding of the geological history of the area.

\section{METHODS}

The Paleogene and the more compacted Neogene sediments were dried in an oven and then soaked in a dilute solution of sodium hexametaphosphate. All samples were washed on a $63 \mu \mathrm{m}$ ( $230 \mathrm{mesh})$ sieve, dried, and then split on a $125 \mu \mathrm{m}$ (120 mesh) sieve. Only the fraction

\footnotetext{
${ }^{1}$ Roberts, D. G., Schnitker, D., et al., Init. Repts. DSDP, 81: Washington (U.S. Govt. Printing Office).

2 Addresses: (Murray) University of Exeter, North Park Road, Exeter EX4 4QE, United Kingdom; (Weston) Stratigraphic Services International (UK) Limited, Tannery Housc, Tannery Lane, Send, Woking GU23, 7EF, United Kingdom.
}

Table 1. Position of sites, Leg 81 .

\begin{tabular}{lcccc}
\hline Hole & Latitude & Longitude & $\begin{array}{c}\text { Depth of } \\
\text { water }(\mathrm{m})\end{array}$ & $\begin{array}{c}\text { Penetration } \\
(\mathrm{m})\end{array}$ \\
\hline 552 & $56^{\circ} 02.56^{\prime} \mathrm{N}$ & $23^{\circ} 13.88^{\prime} \mathrm{W}$ & 2301 & 314.0 \\
$552 \mathrm{~A}$ & $56^{\circ} 02.56^{\prime} \mathrm{N}$ & $23^{\circ} 13.88^{\prime} \mathrm{W}$ & 2301 & 183.5 \\
553 & $56^{\circ} 05.32^{\prime} \mathrm{N}$ & $23^{\circ} 20.61^{\prime} \mathrm{W}$ & 2329 & 9.0 \\
$553 \mathrm{~A}$ & $56^{\circ} 05.32^{\prime} \mathrm{N}$ & $23^{\circ} 20.61^{\prime} \mathrm{W}$ & 2329 & 682.5 \\
$553 \mathrm{~B}$ & $56^{\circ} 05.32^{\prime} \mathrm{N}$ & $23^{\circ} 20.61^{\prime} \mathrm{W}$ & 2329 & 33.5 \\
554 & $56^{\circ} 17.41^{\prime} \mathrm{N}$ & $23^{\circ} 31.69^{\prime} \mathrm{W}$ & 2576 & 76.0 \\
$554 \mathrm{~A}$ & $56^{\circ} 17.41^{\prime} \mathrm{N}$ & $23^{\circ} 31.69^{\prime} \mathrm{W}$ & 2574 & 209.0 \\
555 & $56^{\circ} 33.70^{\prime} \mathrm{N}$ & $2^{\circ} 46.93^{\prime} \mathrm{W}$ & 1666 & 964.0 \\
\hline
\end{tabular}

$>125 \mu \mathrm{m}$ was examined. Counts of at least 100 benthic individuals were made except when the faunas were very sparse. The planktonic to benthic ratio is based on a count of at least 100 individuals.

Altogether 17,628 benthic foraminifers have been mounted from the 162 fossiliferous samples studied. The stratigraphic assignment of each sample is based on the biostratigraphy determined from the plankton (nannoplankton, planktonic foraminifers, radiolarians, and diatoms - see the site chapters, this volume).

The determination of the diversity index $(\propto)$ is based on the graph given by Murray $(1973$, p. 9) following the method of Williams (1964).

Computer analysis was carried out using $\mathrm{Q}$ mode varimax factor analysis.

\section{CRITERIA FOR INTERPRETING FOSSIL ASSEMBLAGES}

Knowledge of the ecology and distribution of modern benthic foraminifers provides the comparative basis for interpreting the paleoecology of fossil assemblages. Although there is a large body of data on shelf and marginal marine environments, the foraminiferal assemblages of the slope and deep ocean are much less well known (Murray 1973; Boltovskoy and Wright, 1976; Douglas and Woodruff, 1981). This clearly limits the paleoecological interpretation of fossil oceanic deposits.

There are numerous problems in the study of modern oceanic benthic foraminifers:

Abundance. In sediments from above the lysocline, planktonic foraminifers outnumber the benthic forms by at least 99 to 1 . Thus, picking an assemblage of $>100$ individuals is very time consuming. Furthermore, the proportion of living forms is very low indeed. The low 


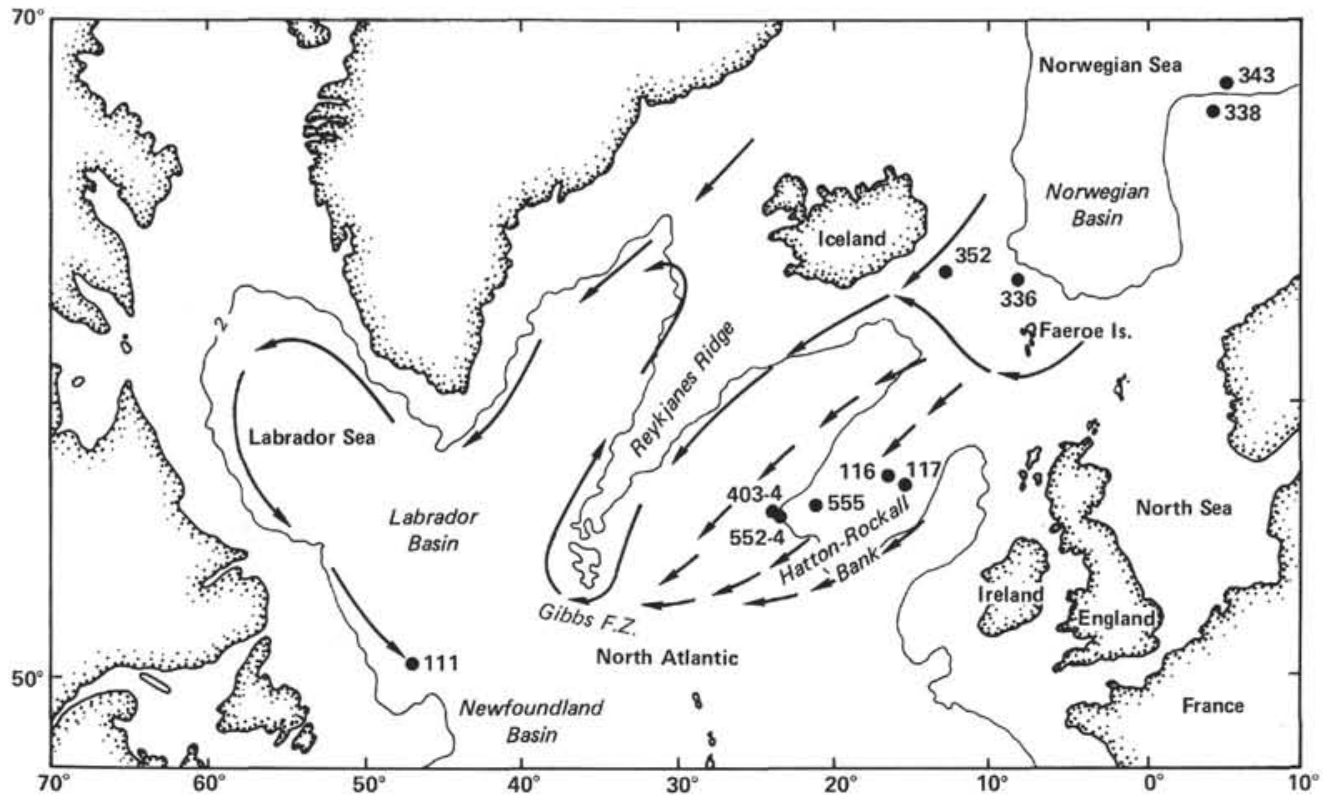

Figure 1. Location of Sites 552 to 555, other DSDP sites used in discussion, and movement of Norwegian Sea Overflow Water into the North Atlantic. Long arrows based on Worthington (1970); short arrows based on Alvinerie et al. (1976, after Berthois and Auffret).

sedimentation rate results in dead tests swamping the living. Further, because of the generally low fertility of oceanic bottom water, the density of living individuals is commonly low anyway.

Live versus dead. It follows that almost all studies of deeper water benthic foraminifers are based on dead assemblages. These are bound to differ from the living assemblages from which they are drawn because of production differences between species and postmortem changes such as mixing as a result of bioturbation or transport (Murray, 1976a). Since the distribution of individual living species on a scale of centimeters is likely to be patchy, with the location of patches varying with time, the diversity of the dead assemblages will be much higher than that of the living assemblages from which they are drawn. A 1-cm-thick sample from an area having a $2 \mathrm{~cm} / 1000$ yr. sedimentation rate will represent 500 years and therefore many generations of foraminifers.

Correlation with ecological parameters. In ecological studies it is normal practice to try to explain distribution patterns with respect to limiting parameters. There are particular problems in trying to do this for deeper water foraminifers.

Physical and chemical oceanographers have recognized that there are different water masses in the oceans, each having its own source, its own direction of movement, and its own physical and chemical attributes. However, the differences are often small: salinity may vary by a few decimal points in parts per thousand; temperature differences may be fractions of a degree. An essentially unanswered question is whether benthic foraminifers are really able to discriminate such small differences. These water mass measurements are not usually made at the sediment/water interface but some distance above, so are they truly representative of the environment in which the foraminifers live? It may not be so for such parame- ters as dissolved oxygen and $\mathrm{CaCO}_{3}$ availability. The most easily measured and most obviously changing parameter is water depth, but most other environmental parameters are interrelated with depth. However, in the Norwegian-Greenland Sea, Belanger and Streeter (1980) found nonvarying physical and chemical water parameters at depths greater than $1500 \mathrm{~m}$ although the benthic foraminifers showed marked assemblage changes. This led them to observe that, "Under such situations of an overall stable environment, it appears that the deep-sea benthos exhibit a sensitivity to parameters we are not used to looking at and that affect species relative and absolute abundance."

The concept of limiting parameters is that each species has its own particular tolerance range for each factor. When any one factor exceeds the tolerance limit for that species, local extinction will occur. In some cases two or more interrelated factors control local extinctions. It follows that no single limiting factor will be responsible for causing local extinction.

Faunal changes through time. It is now known that there have been major changes in the distribution of deepsea benthic foraminifers since the last glacial maximum. The changeover in the oceans proceeded from the top downwards at depths greater than $2500 \mathrm{~m}$ in the western North Atlantic and was a diachronous event (Schnitker, 1979b; Streeter and Lavery, 1982). At individual core sites certain species were more abundant during the glacial event than they are now, e.g., Uvigerina peregrina, $U$. canariensis; others are now more abundant, e.g., Hoeglundina elegans, Pyrgo murrhina, Oridorsalis umbonatus, Osangularia rugosa, and Epistominella exig$u a$. However, the emphasis is on changes in abundance; no new species have been introduced and none has become totally extinct. Perhaps the most significant aspect is that these changes are most readily correlated with 
changes in water masses and their circulation rather than just with depth.

Published Work. The most recent review of modern deep sea benthic foraminiferal distributions is that of Douglas and Woodruff (1981). Although many deep water forms are cosmopolitan, there are differences in distributions not only between but also within oceans. For the interpretation of Rockall material, comparative data from the North Atlantic have been used.

Phleger et al. (1953) have provided much information on distributions in the whole North Atlantic. This, together with data from additional samples, has been subjected to computer analysis by Streeter (1973), while Schnitker (1974) presented new data from the western part only. Both authors found a good correlation between assemblages and water masses. On a regional scale the water masses are transgressive. Schnitker (1980) summarized the relationship for the North Atlantic (Table 2).

The named species are dominant and are accompanied by many associate species. Most species are present in more than one water mass.

Streeter and Lavery (1982), in a study of the continental slope and rise between Cape Hatteras and Tail of the Banks, found that four associations are closely related to depth. Taking data from Miller and Lohmann (1982) for Cape Cod, and from Schafer and Cole (1982) for Newfoundland, it can be seen that this depth-related distribution extends over a great distance (Table 3 ). The Uvigerina assemblage is absent off Newfoundland but the Hoeglundina assemblage starts at the same depth. However, with the exception of the study by Schafer and Cole (1982), all these papers lack details of the assemblages as a whole, and they are therefore difficult to apply to the fossil record. A further complication is that certain species common in the Rockall area appear to be absent from the western Atlantic, e.g., Bulimina alazanensis, Cibicidoides kullenbergi, and Triloculina frigida (Culver and Buzas, 1980, listing consulted).

It should also be noted that the foraminiferal assemblages of the Labrador Sea are dominated by large numbers of agglutinated individuals (Phleger, 1952; Schafer and Cole, 1982) and in this respect are very different from those of the Rockall area.

\section{Deep Water Benthic Foraminiferal Assemblages from the Recent NE Atlantic}

\section{Contribution by J. F. Weston}

Work in progress on Recent benthic foraminiferal faunas from the NE Atlantic has revealed the existence of a very diverse foraminiferal fauna. The following species

Table 2. Water masses and foraminiferal faunas of the North Atlantic (based on Schnitker, 1980).

\begin{tabular}{llc}
\hline \multicolumn{1}{c}{ Water mass } & \multicolumn{1}{c}{ Western } & \multicolumn{1}{c}{ Eastern } \\
\hline Antarctic Bottom Water (AABW) & O. umbonifera & O. umbonifera \\
Arctic Bottom Water (ABW) & E. exigua & - \\
North Atlantic Deep Water (NADW) & E. exigua & P. wuellerstorfi \\
Lower & U. peregrina & U. peregrina \\
Upper &
\end{tabular}

Table 3. Distribution of benthic foraminiferal assemblages on the continental margin of North America. (Data from Streeter and Lavery, 1982; Miller and Lohmann, 1982; and Schafer and Cole, 1982.)

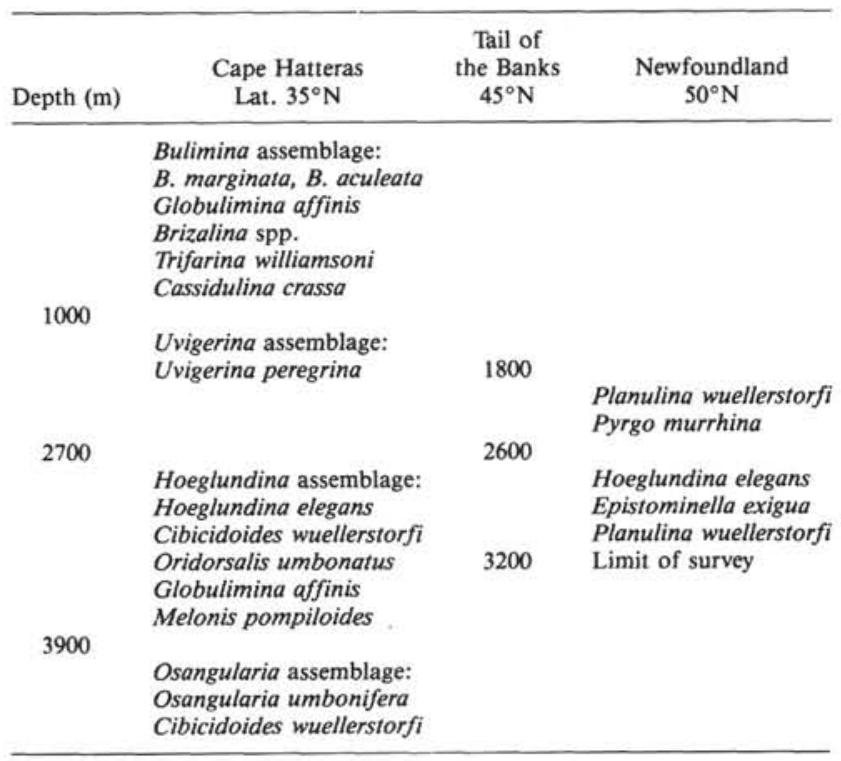

show some depth restriction, especially within the area from $50-60^{\circ} \mathrm{N}$ latitude:

Bolivina aff. B. thalmanni: $1500-3000 \mathrm{~m}$, but is most important in samples from $<2000 \mathrm{~m}$, especially on the eastern slope of the Reykjanes Ridge.

Bulimina aculeata: $1500-3000 \mathrm{~m}$ : Particularly important in samples from the eastern slope of the Reykjanes Ridge.

Bulimina alazanensis: $1000->4000 \mathrm{~m}$ : Not common in Recent sediments from the Rockall-Reykjanes Ridge area, and more abundant in samples from south of $50^{\circ} \mathrm{N}$. It is absent from core-top samples from Sites 552-555, but is present in the Neogene of these sites.

Cibicidoides kullenbergi: $>1500 \mathrm{~m}$ : Rare in Recent sediments north of $50^{\circ} \mathrm{N}$, but abundant in samples south of this at depths between $3500 \mathrm{~m}$ and $4200 \mathrm{~m}$.

Epistominella exigua: $1500 \mathrm{~m}->5000 \mathrm{~m}$ : Probably the most common species in the deep waters of the NE Atlantic. It often makes up $20 \%$ of an assemblage in samples from depths of $2500-3500 \mathrm{~m}$.

Melonis pompilioides: Consistent upper depth limit of 2000-2200 m, with rare shallower occurrences.

Oridorsalis umbonatus: $1500->5000 \mathrm{~m}$ : Maximum abundances of this species occur at $>2500 \mathrm{~m}$.

Planulina wuellerstorfi: Consistent upper depth limit of 1000-1200 m, but most abundant at depths $>2000 \mathrm{~m}$.

Sigmoilopsis schlumbergeri: $500-3500 \mathrm{~m}$, but when abundant, the depth is always $<2200 \mathrm{~m}$.

Triloculina frigida: $1500-3000 \mathrm{~m}$. This species is particularly characteristic of areas north of $50^{\circ} \mathrm{N}$.

With the exception of $B$. alazanensis, all these species are present in core-top samples from Sites 552-555 (depth range 1659-2576 m). With the exception of $B$. aculeata, $B$. alazanensis, and $T$. frigida, they are also recorded from the Norwegian-Greenland Sea (Belanger and Streeter, 1980. O. tener $=$ O. umbonatus, Cibicides wueller- 
storf $i=P$. wuellerstorf $i$. However, $T$. frigida does occur rarely in samples from the Norwegian-Greenland Sea (Belanger, pers. comm. to J. W. Murray).

Two other individual species show relationships with external factors:

Brizalina subaenariensis: Occurs in submarine canyon areas and regions of organic-rich deltaic sediments (Lutze, 1980). It has been interpreted by Sen Gupta et al. (1981) as indicative of localized upwelling, and in the NE Atlantic it appears to have a distribution related to areas of rich food supply.

Melonis barleeanus: Occurs throughout the NE Atlantic, but is particularly abundant in areas where there are active bottom currents.

Qualitative data from the Recent samples have been subjected to Q-mode varimax factor analysis. Four out of ten assemblages derived from this analysis are important in open ocean areas of the NE Atlantic north of $40^{\circ} \mathrm{N}$. The major species components of the factor assemblages are shown in Table 4, and the Recent distribution of these assemblages is shown in Figure 2. (The factor distribution is drawn up from those factors which record loadings of 0.5 to 1.0 or -0.5 to -1.0 in each sample.)

The factor which is most important in samples from $1500-3000 \mathrm{~m}$ in the area north of $50^{\circ} \mathrm{N}$ in the Recent
Table 4. Modern assemblages represented by varimax factors (factor scores $\times 100$ ).

Varimax factor 1

$\begin{array}{ll}50.4 & \text { Globocassidulina subglobosa } \\ 50.3 & \text { Planulina wuellerstorfi } \\ 37.1 & \text { Oridorsalis umbonatus } \\ 34.2 & \text { Cibicidoides kullenbergi } \\ 22.3 & \text { Epistominella exigua } \\ 21.1 & \text { Complex agglutinants }\end{array}$

Varimax factor 4

-86.0 Epistominella exigua

-25.7 Hoeglundina elegans

-22.2 Bolivina spp./Brizalina spp./Fursenkoina spp.

Varimax factor 8

-60.6 Globocassidulina subglobosa

-50.9 Osangularia rugosa

-29.3 Bolivina spp./Brizalina spp./Fursenkoina spp.

-20.7 Uvigerina spp.

Varimax factor 10

-47.3 Pullenia spp.

-44.2 Astrononion spp./N. iridea

-31.0 Bolivina spp./Brizalina spp./Fursenkoina spp.

-21.0 Bulimina spp./Francesita spp.

-19.9 Uvigerina peregrina

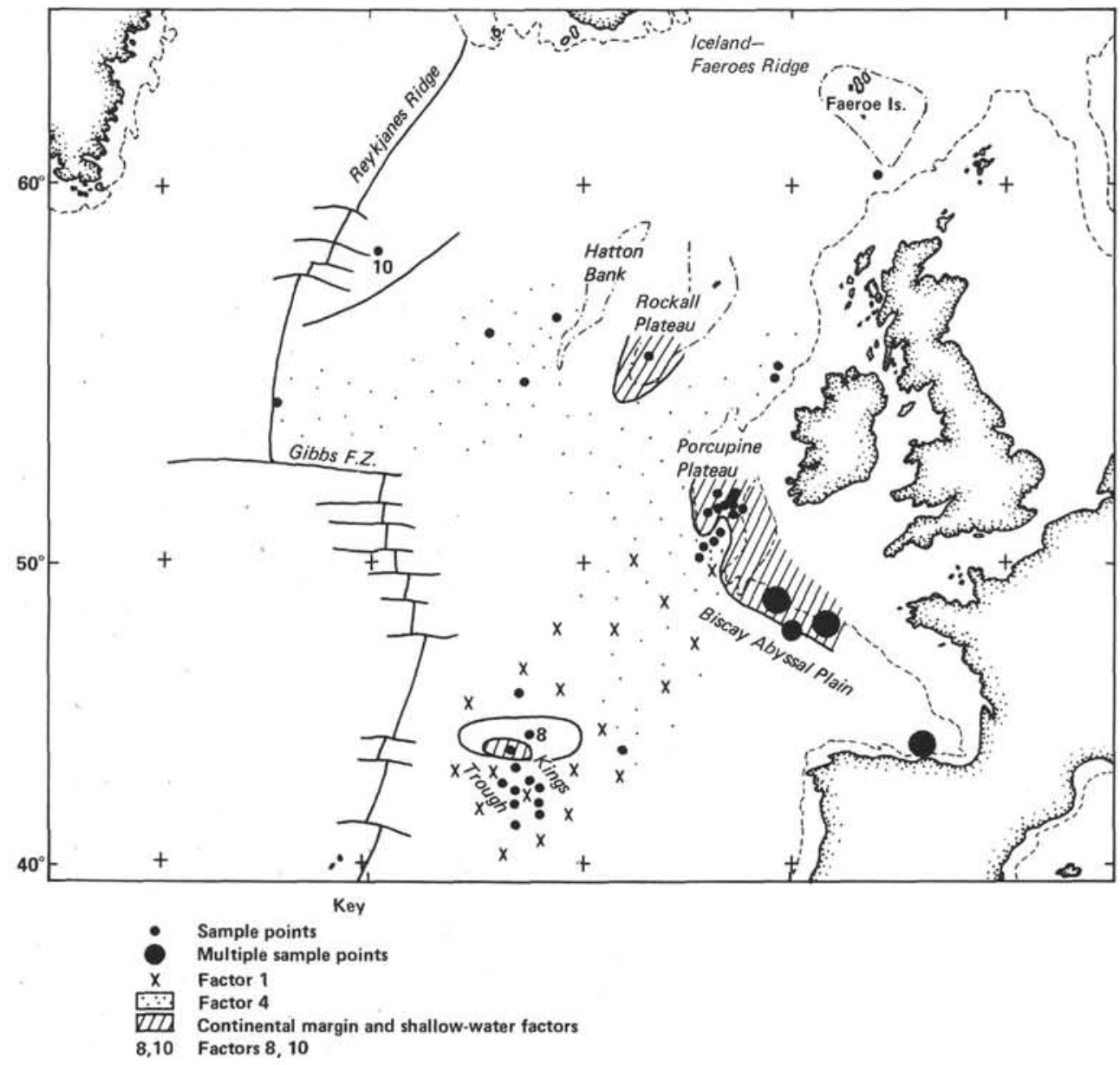

Figure 2. Distribution of Q-mode varimax factor modern assemblages in the northeast Atlantic. 
North Atlantic is factor 4. This represents an assemblage dominated by $E$. exigua. Hydrographic profiles from the Erika Dan cruise (Worthington and Wright, 1970) indicate that these samples (Fig. 2) are taken in areas overlain by Labrador Sea Water and Norwegian Sea Overflow Water.

The true North Atlantic Deep Water (NADW) is composed of Norwegian Sea Overflow Water mixed with considerable quantities of less saline Denmark Straits Overflow Water (Worthington, 1976). The Norwegian Sea Overflow Water present in this area of the Northeastern Atlantic is referred to by some authors as "Northeast Atlantic Deep Water" (NEADW) (e.g., Lonsdale and Hollister, 1979). NEADW forms an upper layer of the NADW (s.1.) which may be concentrated toward the eastern margin of the European Basin. The factor 4 assemblage shows a distribution in the Recent NE Atlantic that is very similar to the distributions of Labrador Sea Water and NEADW.

South of $50^{\circ} \mathrm{N}$, samples from $>3500 \mathrm{~m}$ are characterized by factor 1 assemblages. This is a more diverse assemblage than that represented by factor 4 (see Table 4) and is similar to assemblages related to the distribution of NADW in other areas (e.g., Lohmann, 1978). Hydrographic data for areas where factor 1 shows the highest loadings indicate that, at the depths of these samples, the bottom water mass is probably NADW (s.s.) (U.S. National Oceanographic Data Center Files).

Factors 8 and 10 have relatively restricted occurrences as the important factors in a sample. Factor 8 is important only at $2000-2800 \mathrm{~m}$ on the slopes of seamounts. Factor 10 is important only in one sample from $2353 \mathrm{~m}$ on the eastern slope of the Reykjanes Ridge. It may be related to the relatively high salinity southward flow of Norwegian Sea Overflow Water which occurs at this depth in this area (Worthington, 1976).

Therefore, these varimax factor assemblages appear to have distributions related to the distribution of bottom water masses in the Recent NE Atlantic Ocean. These factor assemblages and their distributions are different from those outlined in Streeter (1973) and Schnitker $(1974,1980)$ and indicate differences between foraminiferal faunas in the northeastern and northwestern Atlantic (possibly enhanced by the examination of different size fractions).

This is confirmed by the comparison of the distributions of individual species in the northwest and northeast Atlantic, Labrador Sea, and Norwegian Sea, already discussed.

\section{Paleogene}

Much is known of the distribution of genera and species and the character of assemblages of modern benthic foraminifers of shelf seas and marginal marine environments (Murray, 1973; Boltovskoy and Wright, 1976). The paleoecological interpretation of such assemblages from the Cenozoic is readily made on such criteria as diversity, triangular plot of the suborders, genera, and planktonic to benthic ratio (Murray and Wright,1974; Murray, 1966).
The recognition of deeper water assemblages is much more difficult for several reasons: The study of modern deep water forms is still in its infancy. There is evidence from the Pacific Ocean that some Paleogene assemblages have changed their depth preferences with time, some migrating into shallower and others into deeper water (Douglas and Woodruff, 1981); in the Rockall Plateau area many of the assemblages are poorly preserved as a result of dissolution.

A useful technique to establish the depth of accumulation of a fossil assemblage overlying ocean crust is to "backtrack" the site, that is, correct the depth for a particular age by subtracting the amount of subsidence and adjusting for sediment thickness (Berger and Winterer, 1974; Douglas and Woodruff, 1981). However, when it is desired to use the foraminifers to determine the depth of deposition, and thereby the subsidence curve, such a technique cannot be used.

Using published data, the assignment of assemblages to different depths has been summarized for the North Atlantic in Figure 3. Although based on comparisons with modern assemblages as well as with other invertebrate groups, such depth assignments are inevitably subjective. Shelf assemblages often contain Anomalinoides howelli as a common component with Cibicidoides alleni, C. succedens, Alabamina obtusa, Pulsiphonina pri$m a$, and Lenticulina spp. The diversity is moderate and the planktonic to benthic ratio varies from 0 near the shore to $40-50 \%$ planktonic forms on the outer shelf.

The slope species include Nonion havanensis, Nuttallides truempyi, and Oridorsalis ecuadorensis, although often not in great abundance. Such species also extend into outer shelf environments. Much of the slope fauna is made up of Nodosaria/Stilostomella spp., Gyroidinoides spp., Cibicidoides spp., and Pullenia spp. Diversity is moderate to high $(\propto>15)$, and the proportion of planktonic tests exceeds $50 \%$ unless affected by dissolution.

\section{QUATERNARY AND NEOGENE ASSEMBLAGES}

The numerical results are listed in Tables 5 to 7 , and the more important general attributes are shown on Figures 4 to 9 . The assemblages are dominated by Rotaliina, with Textulariina and Miliolina playing very subordinate roles. The diversitv is moderate to high with $\propto$ values in the range 10 to 30 . Because of this, individual species do not normally form a very high proportion of the total.

\section{Pleistocene and "Glacial" Late Pliocene}

The best record is that of the HPC of Hole 552A (Cores 1,CC to 9,CC). This "glacial" part of the succession is marked by cyclic changes from white foram nanno oozes to terrigenous oozes. Most of the core-catcher samples came from the white oozes representing interglacial conditions. The diversity shows marked fluctuations from $\propto 30$ in Core $1, \mathrm{CC}$ to $\propto 12$ in Core 9,CC (Fig. 4). The high value in Core 1,CC results in part from the presence of fragile agglutinated species that probably do not withstand fossilization. Common, long-ranging species include Epistominella exigua, Gyroidinoides spp., Oridorsalis umbonatus, Pullenia osloensis, and 


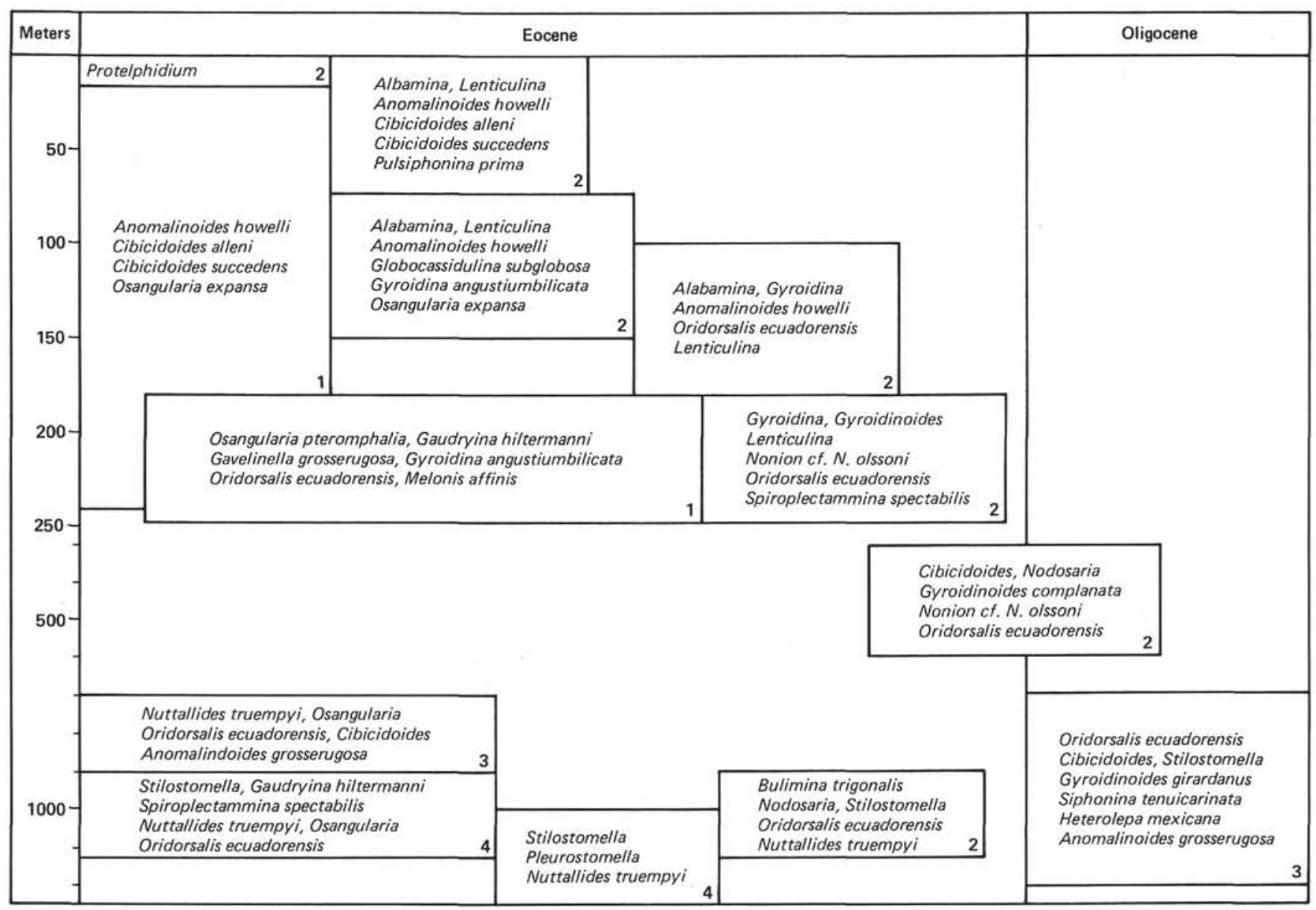

Figure 3. Depth distribution inferred for Paleogene assemblages in the North Atlantic. (1. Berggren, 1974; 2. Murray, 1979; 3. Berggren and Aubert, 1976b; 4. Berggren and Aubert, 1976a.)

Pyrgo murrhina. Three species are restricted to this interval: Cassidulina teretis, which appears in abundance in 2,CC and 9,CC; Triloculina frigida, which is present from 1,CC to 5,CC (i.e., only in the Pleistocene); and Cibicides sp. Melonis pompilioides, which is present in every sample but is rare in the preglacial Neogene. Globocassidulina subglobosa is present only rarely in Core $6, \mathrm{CC}$ but is common in the preglacial Neogene. Cibicidoides kullenbergi is intermittently present in the glacial succession and invariably present below it.

The record at the other sites is far less complete but the pattern of occurrence is essentially the same, except that at Site 555 Melonis pompilioides is rare throughout the whole Neogene because of the shallow depth of the site.

\section{Preglacial Pliocene and Late Miocene}

In Hole 552A the fauna comprises the commonly occurring forms Epistominella exigua, Globocassidulina subglobosa, Gyroidinoides spp., Nodosaria/Stilostomella, Oridorsalis umbonatus, and Planulina wuellerstorfi. Two species are more abundant than they are in the succession above: Cibicidoides kullenbergi and Uvigerina compressa. Ehrenbergina trigona is restricted to this interval while E. serrata and Laticarinina pauperata range down into the middle Miocene. Bulimina alazanensis and Brizalina subaenariensis also extend through this interval.

The commonly occurring forms are also present at the other three sites. C. kullenbergi and $U$. compressa show the same trend in abundance as at Hole 552A.

\section{Middle Miocene}

This part of the succession is best represented in Hole $553 \mathrm{~A}(6, \mathrm{CC}$ to $7, \mathrm{CC})$. The fauna is essentially the same as that of the late Miocene, but Ehrenbergina trigona has not been recorded in this interval. Siphonina tenuicarinata is present in Holes 554A and 555.

\section{Early Miocene}

This is incompletely represented in all four sites. The commonly occurring species are Cibicides sp., Cibicidoides kullenbergi, Globocassidulina subglobosa (especially in Sample 555-24-7, $55 \mathrm{~cm}$ ), Gyroidinoides spp. Melonis barleeanus, Nodosaria/Stilostomella, and Oridorsalis umbonatus. One notable absentee is Planulina wuellerstorfi. Holes 552A, 553A, and 555 have Siphonina tenuicarinata, while Alabamina sp. is abundant and Oridorsalis ecuadorensis is present in Hole 553A. 
Table 5. Benthic foraminiferal census data, Hole 552A, Neogene.

\begin{tabular}{|c|c|c|c|c|c|c|c|c|c|c|c|c|c|c|c|c|c|c|c|c|c|c|c|c|c|c|}
\hline \multirow[b]{2}{*}{ Species } & \multicolumn{7}{|c|}{ eistocene } & \multicolumn{5}{|c|}{$\begin{array}{c}\text { late } \\
\text { Pliocene }\end{array}$} & \multicolumn{5}{|c|}{$\begin{array}{c}\text { early } \\
\text { Pliocene }\end{array}$} & \multicolumn{6}{|c|}{$\begin{array}{c}\text { late } \\
\text { Miocene }\end{array}$} & \multicolumn{3}{|c|}{$\begin{array}{l}\text { middle } \\
\text { Miocene }\end{array}$} \\
\hline & 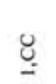 & $\underset{\sim}{\mathbb{N}}$ & $\underset{\sim}{\mathbb{~}}$ & $\underset{f}{\stackrel{U}{*}}$ & $\underset{\sim}{\mathbb{Z}}$ & $\underset{0}{8}$ & $\underset{\sim}{\mathbb{S}}$ & $\underset{\infty}{0}$ & $\underset{\sigma}{0}$ & $\begin{array}{l}\stackrel{U}{0} \\
\stackrel{\Xi}{0}\end{array}$ & $\stackrel{0}{=}$ & $\stackrel{U}{\text { I }}$ & 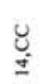 & $\begin{array}{l}\tilde{U} \\
\stackrel{0}{0}\end{array}$ & $\begin{array}{l}\mathcal{U} \\
\Phi\end{array}$ & Ü. & $\underset{\mathbb{N}}{\breve{A}}$ & $\begin{array}{l}\text { U } \\
\text { J }\end{array}$ & 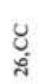 & $\begin{array}{l}\underset{U}{U} \\
\text { d }\end{array}$ & Ü & $\underset{\sim}{\mathbb{j}}$ & 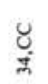 & 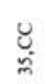 & $\frac{8}{\tilde{N}}$ & $\frac{8}{8}$ \\
\hline trononion guadel & - & - & - & 5 & - & 10 & 3 & - & - & - & - & - & - & - & 5 & - & - & - & 1 & - & 3 & 1 & 5 & - & - & 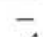 \\
\hline livi & 8 & & $=$ & 4 & & - & 4 & & 1 & 2 & 2 & 5 & 8 & - & 2 & 1 & - & - & - & - & 12 & 1 & - & 1 & - & $\mathrm{H}^{2}$ \\
\hline izaliti & $\tau_{1}$ & 1 & 2 & $=$ & -8 & - & - & & $\bar{z}$ & - & $\bar{z}$ & - & 2 & 18 & 2 & - & 2 & $\frac{1}{3}$ & 2 & - & - & 1 & 1 & 1 & $\overline{-}$ & $\bar{z}$ \\
\hline limi & 4 & - & 2 & $\overline{-}$ & - & & 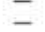 & & $\bar{z}$ & - & $\bar{z}$ & - & $=$ & $\overline{1}_{1}$ & $=$ & - & -2 & - & $\bar{z}$ & $\bar{z}$ & $-{ }^{4}$ & _ & - & $\overline{-}$ & $\bar{z}$ & $\overline{-}$ \\
\hline anensi & - & - & - & - & - & & & & 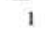 & $=$ & 13 & 1 & , & 19 & 14 & 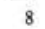 & 8 & 5 & 8 & 13 & 8 & 4 & - & 5 & 13 & - \\
\hline imi & - & 1 & - & 1 & - & & & & - & 2 & & 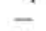 & 8 & & & & & 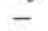 & & & 0 & & 1 & 2 & & \\
\hline ssid & 1 & 4 & & 3 & 3 & 3 & 4 & & - & 2 & - & - & 2 & - & 1 & 1 & & 4 & 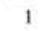 & - & 4 & & & i & - & - \\
\hline ssid: & - & 51 & 14 & 8 & - & & 5 & & 35 & - & & & - & - & - & & & & & & & & & & & \\
\hline icic & 1 & $\therefore$ & 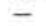 & 1 & & 3 & & 2 & 1 & - & - & - & - & - & - & & & & & & - & & 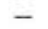 & & 1 & \\
\hline bradyi & 1 & 3 & - & - & - & - & & - & - & - & 1 & - & 3 & 1 & - & & & 1 & & & - & & & 5 & 1 & \\
\hline ticidoides $k$ inll & 3 & - & 1 & - & 2 & 5 & & & - & 4 & 1 & 1 & 2 & 4 & 6 & 4 & & - & 3 & 10 & 1 & 2 & & 5 & 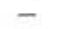 & \\
\hline des ro & - & & - & - & - & 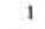 & & & - & - & - & - & - & $\bar{z}$ & - & - & & & 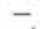 & & - & 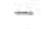 & & & & \\
\hline$s \mathrm{sp}$ & - & 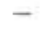 & 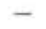 & - & & - & 2 & & - & 1 & 1 & - & - & $\overline{-}$ & - & s & & & 4 & 3 & . & 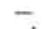 & $e^{2}+$ & 4 & 4 & \\
\hline zent & $-\bar{u}_{4}$ & $\bar{z}_{3}$ & $\bar{z}$ & - & 5 & - & 4 & 7 & ${ }^{2}$ & 2 & $\begin{array}{l}1 \\
2\end{array}$ & $\bar{z}$ & 1 & $=$ & 3 & $\tau_{1}$ & - & - & - & - & 2 & 1 & 6 & 1 & - & 2 \\
\hline rrata & - & -3 & $\bar{I}$ & $=$ & -2 & 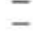 & - & - & $\bar{z}$ & _- & - & $\Xi$ & - & -1 & 1 & - & - & - & -3 & 2 & 3 & - & 1 & _- & $\bar{z}$ & - \\
\hline reen & - & - & - & - & - & & & - & - & 6 & 4 & 2 & - & 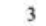 & - & 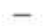 & 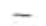 & 1 & - & 3 & 5 & 2 & - & 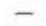 & 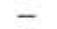 & - \\
\hline Eponi & 2 & $\overline{-}$ & 2 & 2 & - & 1 & & 3 & - & 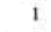 & - & - & 1 & - & - & 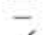 & - & - & - & - & - & - & - & - & - & - \\
\hline Epistoo & 2 & 15 & 5 & 27 & - & 1 & in & 11 & - & - & - & 3 & 5 & 2 & 4 & 6 & 3 & - & 4 & 3 & - & - & - & 1 & 1 & \\
\hline ssuri & 7 & 5 & 6 & 9 & 6 & 4 & 12 & 8 & 5 & 11 & 11 & 8 & 13 & 13 & J & 12 & 10 & 9 & 5 & 3 & 5 & 4 & 5 & 2 & 3 & - \\
\hline ance. & - & - & - & - & - & - & - & I & - & - & - & - & 2 & - & - & - & - & - & 2 & - & - & & - & - & - & - \\
\hline rsen & - & - & 3 & 1 & 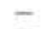 & $s$ & & - & - & - & - & - & - & - & 2 & & - & & 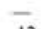 & - & - & - & - & - & - & - \\
\hline boo & - & - & - & - & . & 5 & - & 9 & 10 & - & 4 & 4 & 3 & 5 & 15 & 1 & 9 & 12 & 12 & ${ }^{4}$ & 6 & ${ }^{7}$ & ${ }^{2}$ & 17 & 1 & - \\
\hline th & 2 & 3 & - & $\bar{\tau}$ & 年 & 1 & - & - & 1 & 3 & - & - & - & 1 & & & - & & - & $-\overline{0}$ & - & $\overline{j k}$ & $\overline{-}$ & - & - & $\overline{12}$ \\
\hline & 6 & 2 & 4 & 15 & $0^{\circ}$ & 8 & 6 & 7 & 4 & 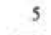 & 3 & 6 & 7 & 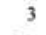 & 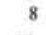 & & 9 & 10 & 10 & 12 & 5 & 15 & 10 & 6 & 11 & 12 \\
\hline egl & - & - & 1 & - & - & - & - & - & - & - & - & - & - & - & - & - & - & - & - & - & - & $\bar{z}$ & $=$ & $\bar{z}$ & $x^{2}+2$ & $\bar{z}$ \\
\hline & 1 & 1 & - & - & - & 1 & 1 & 1 & - & - & - & 1 & - & 1 & 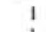 & - & 2 & - & 1 & - & - & - & - & - & 1 & \\
\hline & - & - & - & - & & - & - & - & - & 1 & 2 & 2 & 1 & 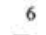 & & & 2 & - & 1 & 1 & 1 & - & & 1 & 1 & - \\
\hline & 1 & 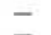 & - & 1 & $=$ & 1 & & & 1 & - & - & 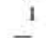 & $i$ & - & & & & & 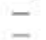 & & 1 & - & & 1 & $\overline{10}$ & 2 \\
\hline & $\begin{array}{l}2 \\
8\end{array}$ & -5 & $2^{2}$ & - & $-\overline{3}$ & 2 & 1 & $\overline{3}$ & 4 & 2 & & - & - & $\overline{1}$ & $\bar{z}$ & & & & I & - & - & & & 1 & & ${ }^{7}$ \\
\hline npiliondes & ${ }_{-}^{8}$ & 5 & 11 & ${ }_{-}^{1}$ & 3 & $2+2+3$ & 1 & 3 & $\begin{array}{l}4 \\
5\end{array}$ & $\overline{8}_{8}$ & & 1 & - & $1+2-1$ & - & & & - & 1 & $\overline{-}_{6}$ & $\overline{-}_{8}$ & & $\tau_{5}$ & $\overline{10}$ & & - \\
\hline tost & 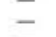 & 1 & - & $\overline{1}_{1}$ & 9 & 15 & 7 & 7 & $\begin{array}{l}5 \\
5\end{array}$ & 11 & 13 & $\begin{array}{r}13 \\
6\end{array}$ & 1 & $\begin{array}{r}3 \\
16\end{array}$ & 1 & & 1 & 1 & 2 & 4 & 6 & $\begin{array}{r}6 \\
6\end{array}$ & 9 & 3 & 1 & 37 \\
\hline & - & 1 & 5 & 2 & - & & 8 & - & - & - & - & - & 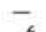 & & 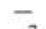 & - & & 0 & 1 & 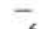 & 5 & 7 & 0 & 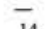 & 1 & - \\
\hline & 6 & 5 & 5 & 5 & 1 & & 8 & - & 7 & 6 & 9 & 7 & 6 & 4 & 7 & & & 8 & 1 & 6 & 5 & 7 & 9 & 14 & 1 & 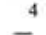 \\
\hline & 1 & $\begin{array}{l}2 \\
9\end{array}$ & & $\tau_{1}$ & $\overline{1}$ & & & & $\overline{-}_{3}$ & 7 & & 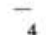 & 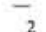 & - & & & & & & & 6 & & & & $\overline{-}_{6}$ & 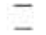 \\
\hline & - & - & & 2 & $i$ & & 8 & 1 & 2 & 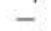 & & 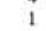 & 4 & $5+2+$ & & & 1 & 2 & & & - & - & & 3 & 1 & - \\
\hline & 3 & 2 & - & - & 1 & & & & & 3 & & & 4 & - & & & - & & & - & & & & & - & \\
\hline & & 2 & 1 & a & & & & 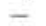 & - & & & & & 1 & - & & & & & & & & & & 9 & \\
\hline & - & & - & & & & & & & & & & & & & & & & & & & & & & - & \\
\hline & & & & & & & & 1 & & & & & & & & & & & & & 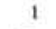 & & & & & \\
\hline & & - & - & - & - & & & 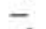 & & & & & - & - & - & & & - & & - & & & & & & \\
\hline & s & 3 & 2 & & & & & & & 10 & 3 & & 4 & & 3 & & & & & & & & & & & \\
\hline & - & - & $\bar{z}$ & - & 3 & - & 1 & - & $=$ & 2 & - & - & - & - & - & - & - & - & - & - & - & & $\pi$ & - & 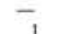 & - \\
\hline & 1 & -1 & $\bar{z}$ & -1 & $\bar{z}$ & - & - & $\underline{-}^{2}$ & $\bar{z}$ & $\bar{z}$ & ${ }_{-}^{\prime}$ & ${ }_{-}^{3}$ & ${ }^{3}$ & $i$ & $\bar{z}$ & - & & 1 & - & $\overline{-}$ & $\exists$ & & $\bar{z}$ & $\overline{-}$ & $\frac{1}{2}$ & - \\
\hline & 1 & 1 & - & & - & & 3 & & & - & - & & 2 & 2 & - & & - & $=$ & 4 & & - & & 1 & - & - & 1 \\
\hline & 1 & - & 1 & & & & & 1 & - & 2 & 年 & & 2 & - & 1 & - & 4 & 2 & 1 & 2 & 3 & - & - & - & - & - \\
\hline & 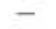 & 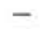 & 1 & - & 1 & & & & - & - & 1 & & 3 & 2 & $\theta^{\circ}+2$ & 3 & 1 & - & & - & - & 1 & 1 & - & - & - \\
\hline & - & 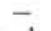 & & & & - & & & & - & & & - & - & - & & & - & - & - & - & - & - & - & 5 & \\
\hline & 1 & 1 & 1 & - & - & 1 & & & 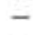 & 1 & & & - & - & - & & & 1 & - & 1 & & 2 & - & - & - & - \\
\hline & 7 & 3 & 1 & & & & & & -5 & & & & & - & - & & & - & 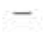 & & - & - & 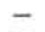 & - & - & \\
\hline & 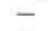 & - & - & & - & 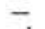 & & & & - & - & - & - & - & & - & - & - & - & - & - & - & - & 5 & - & - \\
\hline & 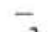 & 1 & 2 & - & & 1 & & & & - & & 3 & 12 & 4 & & & 3 & & & & 3 & 3 & 1 & - & - & \\
\hline & 2 & 1 & 2 & & & 1 & & & & 8 & & - & - & - & & 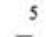 & & 1 & 17 & 19 & 1 & 2 & $\bar{z}$ & 3 & - & \\
\hline & $\overline{13}$ & 8 & 6 & - & & $3+2>$ & - & - & 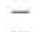 & - & E & - & - & 1 & 3 & - & - & , & 2 & - & 3 & $-{ }_{3}$ & 7 & I & 16 & \\
\hline & 1 & 5 & 6 & - & 7 & 3 & 3 & 4 & - & 2 & 6 & 3 & 5 & 4 & 3 & 3 & 2 & 2 & J & 1 & 3 & 3 & 7 & 4 & 16 & \\
\hline otal & 116 & 133 & III & 104 & 102 & 106 & 109 & 102 & 102 & 109 & 104 & 106 & 148 & 123 & 134 & 104 & 109 & 114 & 115 & 116 & 110 & 104 & 112 & 115 & 131 & 133 \\
\hline Ratio & $\begin{array}{l}46 \\
99\end{array}$ & $\begin{array}{l}34 \\
49\end{array}$ & $\begin{array}{l}31 \\
99\end{array}$ & $\begin{array}{l}30 \\
99\end{array}$ & $\begin{array}{l}38 \\
99\end{array}$ & 99 & $\begin{array}{l}40 \\
99\end{array}$ & $\begin{array}{l}38 \\
99\end{array}$ & $\begin{array}{l}27 \\
49\end{array}$ & 99 & 99 & $\begin{array}{l}36 \\
99\end{array}$ & $\begin{array}{l}42 \\
99\end{array}$ & $\begin{array}{l}42 \\
99\end{array}$ & 49 & 45 & $\begin{array}{l}40 \\
99\end{array}$ & 99 & 99 & 99 & 99 & $\begin{array}{l}35 \\
49\end{array}$ & $\begin{array}{l}36 \\
99\end{array}$ & 99 & 35 & \\
\hline
\end{tabular}

Note: Dash $=$ absent in assemblage count.

\section{Summary}

Those species showing restricted stratigraphic distributions have been plotted in Figure 10. This shows quite clearly that most of the first appearances are at disconformities and, for any given species, the local extinction is variable in time. This may in part result from the small sample size, but very probably local differences in water masses are also responsible for the patchy distributions. It is clear that none of these forms is very reliable as a biostratigraphic marker except in a very crude way. Thus Neogene species that die out with the oncoming of "glacial" conditions include Bulimina alazanensis, Laticarinina pauperata, Ehrenbergina serrata, and E. trigona. The two species that characterize the "glacial" succession are Cassidulina teretis and Triloculina frigida.

\section{Environmental Interpretation}

The criteria for the interpretation of the paleodepths have already been discussed. In this study it has been shown that with few exceptions (e.g., Alabamina sp., Oridorsalis ecuadorensis, Siphonina tenuicarinata), all the species recorded in the Neogene successions are extant either in the Rockall area or elsewhere in the North Atlantic. Furthermore, the main body of species are characteristic of North Atlantic Deep Water. It may therefore be assumed that either NADW has occupied the area throughout the Neogene or that the faunas living in the area have adapted to the introduction of NADW at whatever time it took place.

For depth interpretation it is convenient to assume the long-continued existence of NADW and to apply mod- 
Table 6. Benthic foraminiferal census data, Holes 553, 553A, 554, 554A, Neogene.

\begin{tabular}{|c|c|c|c|c|c|c|c|c|c|c|c|c|c|c|c|c|c|c|c|c|c|c|c|c|c|}
\hline \multirow[b]{3}{*}{ Species } & \multirow{3}{*}{$\frac{\begin{array}{c}\text { Pleist. } \\
\text { Hole } \\
553\end{array}}{\tilde{U}}$} & \multicolumn{2}{|c|}{$\begin{array}{c}\text { late } \\
\text { Pliocene } \\
\end{array}$} & \multirow{3}{*}{ 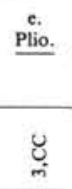 } & \multicolumn{2}{|c|}{$\begin{array}{c}1 . \\
\text { Mio. }\end{array}$} & $\stackrel{\mathrm{n}}{\mathrm{M}}$ & & & $\begin{array}{l}\text { e. } \\
\text { Miocene }\end{array}$ & & & Pleist. & & $\begin{array}{c}\text { e. } \\
\text { Plio. }\end{array}$ & & & & Mio & & & & $\stackrel{r}{M}$ & i. & $\begin{array}{c}\text { e. } \\
\text { Mio. }\end{array}$ \\
\hline & & & & & & $\begin{array}{l}\mathrm{H} \\
55\end{array}$ & & & & & & & & & $\begin{array}{c}\text { Hole } \\
554\end{array}$ & & & & & & & $\begin{array}{l}\text { Hole } \\
554 \mathrm{~A}\end{array}$ & & & \\
\hline & & 0 & $\underset{i}{\text { in }}$ & & $\underset{8}{8}$ & $\underset{n}{0}$ & 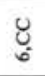 & $\stackrel{8}{\circ}$ & $\underset{\infty}{0}$ & $\frac{2}{\alpha}$ & $\begin{array}{l}2 \\
\text { i }\end{array}$ & $\stackrel{0}{0}$ & $\underset{i}{U}$ & $\underset{\text { i }}{\text { U. }}$ & $\underset{\text { U. }}{\text { U. }}$ & $\underset{0}{0}$ & $\underset{\sim}{\circ}$ & $\underset{\infty}{0}$ & $\stackrel{R}{I}$ & U્త & i & $\underset{i}{U}$ & $\stackrel{\infty}{\infty}$ & $\underset{i}{0}$ & $\begin{array}{l}0 \\
\mathfrak{j}\end{array}$ \\
\hline Alabamina $\mathrm{sp}$ & - & - & - & - & - & - & - & - & 21 & 31 & 7 & - & - & - & - & - & - & - & - & - & - & - & - & - & - \\
\hline $\begin{array}{l}\text { Astrononion guadelupae } \\
\text { Boliving } \mathrm{cf} \text { B. thalmanni }\end{array}$ & - & $\bar{z}+x+1+x$ & - & $\frac{1}{4}$ & - & $=$ & - & $\frac{1}{2}$ & ${ }_{2}^{11}$ & 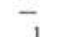 & $\bar{z}+$ & 1 & 1 & 2 & $=$ & 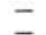 & $=$ & 2 & 14 & 1 & 17 & $?$ & 3 & $\overline{-}$ & - \\
\hline Brizalina subaenariensis & - & $\bar{z}$ & 2 & 1 & 1 & $\bar{s}_{5}$ & ${ }_{4}^{3}$ & ${ }_{2}^{2}$ & $2^{2}$ & 1 & $=$ & $z$ & $z$ & $\bar{z}$ & $\overline{2}_{2}$ & - & $\bar{z}+$ & $=$ & 3 & $\overline{-}$ & $\Xi$ & $\begin{array}{l}3 \\
1\end{array}$ & $\frac{1}{5}$ & $\bar{T}_{3}$ & $\bar{z}+x+x+1$ \\
\hline Brizalina sp. & - & 2 & - & 1 & - & 2 & - & - & - & - & 1 & - & - & - & $i$ & 3 & - & - & 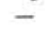 & - & 1 & 4 & - & - & \\
\hline Bulimina aculeat & 1 & - & - & - & - & 1 & - & - & - & - & - & - & - & - & - & - & - & - & - & - & - & - & - & - & - \\
\hline Bulimina alazanensi & - & - & 12 & 1 & 2 & - & 2 & 7 & 1 & 2 & - & - & - & - & 12 & 1 & 9 & 1 & $s$ & 2 & 1 & 2 & 2 & 2 & 1 \\
\hline Bulimina striata & - & - & 1 & - & 3 & - & - & $\mathrm{t}$ & - & 1 & - & - & - & - & - & 2 & - & 1 & - & 1 & 1 & 2 & $\pi$ & - & \\
\hline Cassidulina obtusa & 1 & 2 & - & - & 1 & 1 & - & - & - & - & - & - & - & 2 & - & 3 & 1 & - & - & - & 1 & 3 & 1 & - & - \\
\hline Cassidulina teretis & - & - & - & - & - & - & - & - & - & - & - & 8 & 2 & 1 & - & - & - & - & - & - & - & - & - & - & - \\
\hline Cibicides sp., Cibicidina sp. & - & - & - & - & - & - & - & - & - & - & - & 2 & 3 & 2 & 1 & - & - & - & - & - & - & - & - & - & 13 \\
\hline Cibicidoides bradyi & - & - & - & - & 1 & 2 & - & 1 & 3 & 6 & - & 2 & 1 & 2 & - & - & - & 2 & - & 8 & 3 & - & - & 3 & 4 \\
\hline Cibicidoides kullenbergi & 2 & 1 & 4 & 8 & 1 & 4 & 4 & 1 & - & 13 & 10 & - & - & - & 5 & 1 & 2 & 1 & 3 & 2 & - & - & 5 & 4 & 3 \\
\hline Cibicidoides robertsoniamus & - & - & - & - & - & - & - & - & - & - & - & 1 & 5 & - & 1 & - & - & & - & - & - & - & - & - & - \\
\hline Cibicidoides sp. & 1 & 2 & - & 1 & - & - & - & - & - & & 1 & 1 & - & 2 & - & - & - & 2 & - & - & - & - & - & - & 1 \\
\hline Dentalina sp. & 1 & - & 3 & - & - & - & - & - & - & - & - & - & - & - & - & - & - & - & - & - & - & - & - & - & 1 \\
\hline Eggerella bradyi & 1 & 3 & 2 & 3 & 1 & - & 1 & 5 & - & - & 1 & 4 & 2 & 2 & 2 & 1 & 2 & 1 & 8 & 4 & - & 3 & 1 & - & - \\
\hline Ehrenbergina serrata & - & - & 2 & 2 & 4 & - & - & - & - & - & - & - & - & - & 2 & 1 & 4 & 9 & 1 & 8 & 1 & 2 & - & - & - \\
\hline Ehrenbergina trigona & - & 2 & 2 & 2 & - & - & - & - & - & - & - & - & - & - & - & - & - & 1 & - & - & - & - & - & - & - \\
\hline $\begin{array}{l}\text { Epistominella exigua } \\
\text { Eponides tumidulus }\end{array}$ & 4 & 2 & $2^{2}$ & $\overline{-}+x+3+$ & ${ }^{4}$ & 2 & 1 & 5 & $\bar{z}$ & 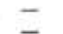 & 2 & 9 & 13 & ${ }^{3}$ & $\bar{z}+$ & 2 & ${ }^{3}$ & 8 & 2 & 8 & 2 & 3 & ${ }^{3}$ & $\bar{z}+$ & $\overline{-}$ \\
\hline Eponides tumidulus & 2 & - & - & -7 & $\tau_{0}$ & - & - & - & - & - & - & - & - & - & - & 5 & - & - & 1 & - & - & 5 & - & - & - \\
\hline Fissurina, Lagena, Oolina & 11 & 7 & 6 & $?$ & 9 & 7 & 1 & 5 & 1 & 12 & 2 & 6 & 6 & 6 & 7 & 5 & 7 & 3 & 5 & 9 & 6 & 5 & 3 & 1 & 2 \\
\hline $\begin{array}{l}\text { Francesita adven } \\
\text { Fursenkoing schr }\end{array}$ & $\frac{1}{3}$ & $\bar{z}+$ & $=$ & 1 & $\bar{z}$ & $\bar{z}$ r & $\bar{z}+2+$ & $\bar{z}+$ & $\bar{z}$ & $\bar{z}$ & $\bar{z}$ & 1 & - & $\bar{z}$ & $\bar{z}$ & $\bar{z}+2+1$ & $\bar{z}$ & $\bar{z}+2+$ & $\bar{z}$ & ${ }_{-}^{1}$ & $\bar{z}+$ & $\bar{z}$ & $=$ & $\bar{z}$ & $\bar{z}$ \\
\hline $\begin{array}{l}\text { Fursenkoina scl } \\
\text { Glopocossidulin }\end{array}$ & ${ }_{-3}^{3}$ & 7 & -5 & -5 & -7 & $\overline{16}$ & $-\overline{3}$ & -8 & $\overline{20}$ & $-{ }_{6}$ & $-{ }_{8}$ & 1 & 1 & $=$ & -7 & $\overline{13}$ & -8 & $\overline{-}_{8}$ & $\overline{13}$ & $\overline{-}_{2}$ & $\overline{-}_{6}$ & 70 & $-_{4}$ & $\overline{12}$ & $\bar{z}+$ \\
\hline $\begin{array}{l}\text { Globocassidulina subgl } \\
\text { Gulobuling Gutuling }\end{array}$ & - & i & $\begin{array}{l}5 \\
2\end{array}$ & $\begin{array}{l}3 \\
2\end{array}$ & ${ }_{-}^{7}$ & 16 & 3 & 8 & 20 & 6 & $\begin{array}{l}8 \\
5\end{array}$ & $=$ & - & - & 7 & 13 & 8 & 8 & $\begin{array}{l}13 \\
3\end{array}$ & 2 & 6 & 10 & ${ }^{4}$ & 12 & $\bar{z}$ \\
\hline $\begin{array}{l}\text { Globulina, Guttulina } \\
\text { Gymidinoides }\end{array}$ & $\begin{array}{l}2 \\
4\end{array}$ & $\frac{1}{10}$ & $\begin{array}{l}2 \\
8\end{array}$ & $\begin{array}{c}2 \\
15\end{array}$ & $\bar{\tau}_{1}$ & $\frac{1}{7}$ & $\overline{19}$ & $\frac{1}{17}$ & $1_{10}^{2}-1-x-1$ & 1 & $\begin{array}{l}5 \\
9\end{array}$ & -7 & 2 & 2 & 2 & $\overline{20}$ & 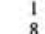 & $\frac{1}{5}$ & $1^{3}$ & $\tau_{5}$ & $\overline{13}$ & $\overline{15}$ & -7 & 2 & $\overline{12}$ \\
\hline & 5 & - & - & - & - & - & - & 17 & 10 & $3^{7}$ & & -7 & 9 & 4 & 10 & & ${ }^{8}$ & 5 & 11 & 5 & ${ }_{-}^{13}$ & 15 & ${ }^{7}$ & ${ }^{7}$ & 12 \\
\hline $\begin{array}{l}\text { Hoogh } \\
\text { Karrer }\end{array}$ & - & $\overline{-}$ & $=$ & $=$ & $=$ & $=$ & $=$ & $=$ & $\overline{-}$ & $=$ & - & $=$ & $\overline{-}$ & - & $\bar{z}_{3}$ & - & $=$ & $\bar{z}$ & $\overline{-}$ & $=$ & $\overline{1}_{1}$ & $\bar{i}_{1}$ & $-{ }_{2}$ & - & - \\
\hline Laticarinina pauperata & - & 2 & 3 & 1 & 1 & 2 & 1 & $=$ & $=$ & $z$ & & - & $=$ & - & 1 & - & 3 & 1 & - & 2 & 1 & $i$ & $\begin{array}{l}2 \\
4\end{array}$ & 1 & 1 \\
\hline Lentic & - & 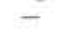 & 1 & - & - & - & 2 & 2 & - & - & & - & - & 1 & - & & 2 & - & - & & & - & 1 & - & 3 \\
\hline Melo & 1 & - & - & - & 1 & - & - & 5 & 3 & 11 & 10 & 2 & - & - & 2 & - & - & - & - & - & 1 & 1 & 3 & 5 & 3 \\
\hline telor & 4 & 2 & - & - & & - & - & - & - & & - & 1 & 5 & 2 & 2 & 2 & - & - & - & 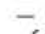 & - & - & & 1 & - \\
\hline elot & - & 13 & 24 & 4 & 5 & 5 & 9 & 9 & - & - & & - & 4 & 1 & 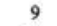 & 6 & 1 & 7 & 12 & 6 & 8 & 14 & 2 & 5 & 8 \\
\hline o & - & 14 & 10 & 16 & 4 & 7 & 11 & 20 & 14 & & 18 & & $s$ & 14 & 17 & 5 & 7 & 11 & & 4 & 5 & 10 & 1 & 21 & 20 \\
\hline & - & - & - & - & 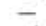 & & - & - & & t & i & & - & - & - & & - & - & & & - & - & & & - \\
\hline & 2 & 11 & 2 & $?$ & 1 & 11 & 5 & 13 & 4 & 6 & 4 & 14 & 5 & 6 & 6 & 13 & 11 & 7 & 1 & 1 & 1 & 6 & 8 & 6 & 3 \\
\hline & 3 & 2 & 4 & 2 & 3 & 4 & - & - & - & - & - & o & 1 & & & & 3 & & - & - & & - & & & 2 \\
\hline & 2 & 9 & 11 & 6 & 7 & 14 & 11 & 17 & - & - & - & 16 & 22 & 17 & 17 & & 3 & 8 & & 4 & 10 & 7 & & 23 & - \\
\hline & - & 2 & 6 & 5 & 2 & 1 & 2 & - & 6 & 6 & 1 & 1 & 1 & 1 & & 2 & 2 & 2 & & ; & 7 & 2 & & 4 & 5 \\
\hline Pulle & 2 & - & 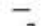 & 2 & & 3 & 2 & 2 & 3 & & - & 6 & 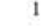 & - & & 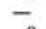 & - & - & - & 3 & & - & & 1 & 5 \\
\hline & - & 4 & 7 & 5 & 15 & 20 & 4 & 12 & 10 & 21 & 7 & 2 & - & - & 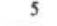 & 9 & 7 & 7 & 7 & & 14 & 7 & 12 & 2 & - \\
\hline & 20 & $r$ & 1 & 6 & . & & 7 & 3 & 6 & 2 & & 8 & 2 & 5 & - & 8 & 8 & 12 & 9 & 11 & 5 & & - & 4 & 4 \\
\hline & - & - & - & - & + & 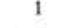 & - & - & - & - & & - & 1 & - & - & ? & - & & $\pi$ & & 1 & 2 & - & & $\bar{z}$ \\
\hline & - & $\frac{7}{2}$ & 5 & $\bar{z}$ & $\bar{z}$ & $\bar{z}$ & 1 & ${ }^{3}$ & $\bar{z}$ & - & & 3 & - & 8 & - & 2 & $\bar{z}$ & & 1 & & 1 & & 1 & & - \\
\hline P & $\overline{-}$ & $2^{2}$ & $=$ & $=$ & 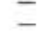 & $\bar{z}$ & $\bar{z}$ & $\bar{t}_{1}$ & $\bar{z}$ & $\bar{z}$ & $\tau_{1}$ & $\begin{array}{l}4 \\
2\end{array}$ & $T_{1}$ & -6 & $\overline{1}_{1}$ & 1 & $\bar{z}$ & $\tau_{1}$ & $\bar{i}_{1}$ & $\bar{z}$ & 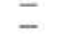 & $\tau_{2}$ & $=$ & - & $\bar{z}$ \\
\hline & - & 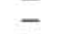 & - & - & 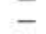 & $=$ & $=$ & 2 & $\overline{-}$ & - & $i$ & - & - & - & - & - & 1 & - & - & - & - & - & - & - & - \\
\hline rger & - & - & - & - & - & - & 1 & 2 & - & - & - & 1 & 1 & 7 & - & - & - & - & 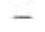 & - & 2 & - & 1 & - & - \\
\hline & - & - & - & - & - & - & - & - & . & 2 & 1 & - & - & - & - & - & - & - & - & 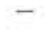 & - & - & - & - & - \\
\hline & - & 2 & 5 & 4 & & - & - & 2 & - & - & 1 & & - & - & 1 & 1 & 1 & 1 & 1 & 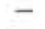 & - & $=$ & - & - & - \\
\hline & 2 & - & - & - & - & - & - & 3 & - & - & - & & - & - & - & - & - & - & - & - & - & - & - & - & - \\
\hline & - & - & 1 & 2 & - & - & - & - & - & - & - & & - & - & - & - & - & - & - & - & - & - & - & - & - \\
\hline$S_{T}$ & - & - & - & - & - & - & - & - & 3 & 3 & 4 & - & - & -7 & - & & & - & - & - & - & - & - & - & 1 \\
\hline & 5 & - & - & - & - & - & - & - & - & - & - & 1 & - & - & - & - & 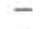 & - & - & - & - & 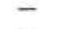 & - & $\overline{1}$ & - \\
\hline & - & - & $-{ }_{6}$ & - & 1 & -5 & - & - & - & - & - & - & $\overline{-}$ & 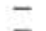 & $\bar{s}$ & - & - & - & 1 & - & - & - & $\overline{1}$ & 1 & - \\
\hline & - & -8 & 6 & 6 & 5 & 5 & 6 & - & 1 & 4 & - & 1 & - & - & 15 & 1 & 4 & 5 & - & 8 & $\bar{z}$ & 3 & 11 & 6 & - \\
\hline$U_{V}$ & 2 & 8 & 9 & - & 3 & - & - & - & - & - & 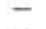 & 6 & 3 & 3 & 3 & - & - & 1 & 2 & - & $\bar{z}$ & $\overline{-}$ & - & - & - \\
\hline $\begin{array}{l}V_{a} \\
V_{u}\end{array}$ & 14 & $\bar{z}$ & $\bar{z}$ & $\bar{z}$ & $\bar{z}$ & - & $\bar{z}$ & $\bar{z}$ & $\bar{z}$ & - & - & - & $\bar{z}$ & $\bar{z}$ & - & - & $\bar{z}$ & $\bar{z}$ & - & $\bar{z}$ & $\bar{\pi}$ & $\overline{=}$ & $=$ & $\bar{z}$ & $\overline{-}$ \\
\hline $\begin{array}{l}\text { Tuvulina pernariula } \\
\text { Unidentified }\end{array}$ & $\bar{s}_{5}$ & $\tau_{1}$ & $\tau_{4}$ & $=$ & $\overline{-}$ & $\frac{1}{2}$ & $\tau_{1}$ & $\overline{2}_{2}$ & $\overline{10}$ & $\overline{20}$ & $\begin{array}{l}1 \\
6\end{array}$ & $\tau_{1}$ & $\bar{z}+$ & 7 & $\tau_{5}$ & $=$ & 7 & $\overline{1}_{1}$ & $\overline{1}_{1}$ & 2 & $\overline{3}$ & -2 & $\tau_{3}$ & -4 & -8 \\
\hline Total & 109 & 125 & 150 & 118 & 100 & 132 & 105 & 156 & 132 & 202 & 105 & 132 & 104 & 107 & 152 & 110 & 105 & 110 & 125 & 100 & 112 & 137 & 115 & 119 & 102 \\
\hline Number & 38 & 37 & 41 & 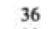 & 3 & 33 & 2 & 4 & 3 & 3 & & 3 & 3 & & 4 & & 3 & 33 & & 9 & 31 & 35 & 31 & 28 & 28 \\
\hline $\begin{array}{l}\text { Ratio planktonic to } \\
\text { benthic }(x: 1)\end{array}$ & 9 & 99 & 99 & 9 & 99 & 99 & 99 & 32 & 24 & 49 & 49 & 99 & 99 & 99 & 99 & 32 & 99 & 99 & 99 & 99 & 99 & 99 & 99 & 99 & 99 \\
\hline
\end{tabular}

Note: Dash $=$ absent in assemblage count.

ern results to the fossil record. The interpretations can then be judged as to whether or not they are reasonable.

The assemblages in Holes 552A, 553A, and 554, 554A are all basically similar in that they are of the Planulina wuellerstorfi type. The commonly occurring species are Cibicidoides kullenbergi, Epistominella exigua, Gyroidinoides spp., Melonis spp., Nodosaria/Stilostomella, Oridorsalis umbonatus, and Pullenia spp. The diversity is moderate to high $(\propto 10-30)$ and so too is the planktonic to benthic ratio (except in a few samples that have been affected by dissolution).

The $P$. wuellerstorfi fauna indicates depths $>1500 \mathrm{~m}$. With the exception of the "glacial" succession in Hole $552 \mathrm{~A}$, Melonis pompilioides is present in low abundance and this may indicate depths $>2000 \mathrm{~m}$, while the general rarity of Sigmoilopsis schlumbergeri suggests depths $>2200 \mathrm{~m}$ (its lower limit of distribution between $50^{\circ}$ and $60^{\circ} \mathrm{N}$ ). Likewise, E. exigua normally forms $>20 \%$ of the assemblage at depths $>2500 \mathrm{~m}$, but only rarely does it approach such high abundance at any of the sites. Thus, from these observations, it appears likely that the depth of deposition of the middle Miocene to Recent in Holes 552A, 553A and 554, 554A was 2200 to $2500 \mathrm{~m}$ (cf. present depths of $2301 \mathrm{~m}, 2329 \mathrm{~m}$, and $2576 \mathrm{~m}$, respectively) (see Figs. 4-6, 8).

The assemblages at Site 555 are also of $P$. wuellerstorfi type with common Brizalina subaenariensis, Bulimina spp., Cibicidoides kullenbergi, Epistominella ex- 
Table 7. Benthic foraminiferal census data, Hole 555, Neogene.

\begin{tabular}{|c|c|c|c|c|c|c|c|c|c|c|c|c|c|c|c|c|c|c|c|c|c|c|c|c|c|c|}
\hline \multirow[b]{2}{*}{ Species } & \multicolumn{2}{|c|}{ Pleist. } & \multirow{2}{*}{$\begin{array}{l}\text { e. } \\
\text { Pl. } \\
\underset{j}{y} \\
\underset{j}{y}\end{array}$} & \multicolumn{13}{|c|}{ late Miocene } & \multicolumn{7}{|c|}{ m. Miocene } & \multicolumn{3}{|c|}{ e. Miocene } \\
\hline & $\underset{-}{\mathscr{Z}}$ & $\underset{i}{\mathbb{i}}$ & & $\underset{8}{8}$ & $\underset{n}{8}$ & $\underset{0}{0}$ & $\underset{\sim}{\mathscr{Z}}$ & $\underset{\infty}{\mathscr{\infty}}$ & $\underset{\sim}{0}$ & 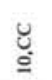 & $\begin{array}{l}\text { U. } \\
=\end{array}$ & $\begin{array}{l}\mathscr{U} \\
\mathbb{\Xi}\end{array}$ & 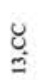 & $\begin{array}{l}\text { U } \\
\text { I }\end{array}$ & $\begin{array}{l}u \\
\underline{u} \\
\underline{v}\end{array}$ & $\begin{array}{l}\underset{U}{0} \\
\underline{0}\end{array}$ & $\stackrel{\text { U }}{\Xi}$ & 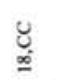 & $\begin{array}{l}\mathscr{U} \\
\stackrel{\alpha}{O}\end{array}$ & ¿্ه & $\underset{\vec{N}}{\tilde{N}}$ & $\begin{array}{l}\mathbb{A} \\
\text { J }\end{array}$ & $\begin{array}{l}\text { U } \\
\text { ì }\end{array}$ & $\begin{array}{l}\tilde{n} \\
\frac{\tilde{a}}{3}\end{array}$ & $\begin{array}{c}\text { U. } \\
\text { d }\end{array}$ & $\begin{array}{l}\text { U } \\
\text { d. }\end{array}$ \\
\hline trononion guadelupae & - & 3 & - & 1 & - & 10 & - & 4 & 21 & - & - & - & 1 & 4 & 4 & 1 & - & - & 2 & - & 1 & 1 & 2 & - & - & - \\
\hline Bolivina ef. $B$, thalmanni & 1 & 1 & $\overline{0}$ & 3 & 4 & 1 & - & - & 3 & 3 & 5 & 3 & - & - & - & - & - & - & 1 & - & - & - & 7 & - & - & - \\
\hline $\begin{array}{l}\text { Brizalina subaenarie } \\
\text { Brizalina }\end{array}$ & $-{ }^{3}$ & - & 22 & $=$ & 3 & $-_{3}$ & - & $\frac{1}{3}$ & 2 & 2 & 2 & 1 & 11 & 3 & 24 & 30 & 3 & 8 & 4 & "II & 1 & $\bar{z}$ & 3 & 5 & 4 & $\bar{z}$ \\
\hline Bulimina aculeata & 14 & $\mathrm{i}$ & - & - & - & - & - & - & -1 & 2 & - & -7 & $2^{2}$ & $\bar{z}$ & -4 & $=$ & 1 & - & $=$ & - & $\overline{-}$ & $\bar{z}$ & $3^{3}$ & -1 & -5 & $z$ \\
\hline Bulimina alazanensis & - & $\mathrm{i}$ & 8 & 5 & 2 & 10 & 6 & 17 & - & 1 & 2 & 1 & - & - & 1 & - & - & - & 1 & 1 & 5 & 1 & 3 & - & - & 8 \\
\hline Bulimina striata & 1 & - & 5 & 4 & is & 1 & 6 & 2 & 2 & 9 & $i$ & 2 & 1 & 5 & $\mathrm{i}$ & 1 & - & 5 & 2 & i & 8 & s & 6 & 2 & 3 & 1 \\
\hline Cassidulina obtusa & 1 & 5 & 1 & 1 & 2 & - & - & - & - & - & - & 2 & - & - & - & 2 & 1 & 2 & $i$ & 4 & - & - & - & - & - & - \\
\hline Cassidulina teretis & 9 & 7 & - & - & - & - & - & - & - & - & - & - & $=$ & $=$ & - & - & - & - & - & - & - & - & - & - & - & - \\
\hline Cibicides sp., Cibicidina sp. & - & - & 1 & 1 & - & - & - & - & - & - & - & - & - & 1 & - & - & - & - & 2 & - & - & 1 & 1 & 12 & 10 & 3 \\
\hline Cibicic & 1 & 2 & 3 & 8 & - & - & 6 & 2 & 4 & 6 & 9 & 3 & - & - & 3 & 1 & 4 & - & 2 & 3 & 3 & $i$ & i & - & 1 & i \\
\hline Cibicio & - & - & 5 & 14 & - & 13 & 6 & 3 & 7 & 3 & 3 & 11 & 4 & 4 & 3 & - & i & 4 & - & 3 & 4 & 2 & 3 & 1 & 5 & 7 \\
\hline Cibici & 3 & 2 & - & 1 & - & - & - & - & - & - & - & - & - & - & - & - & - & - & - & - & - & - & - & - & - & - \\
\hline Cibicidoides sp. & - & - & - & 1 & 1 & - & 2 & - & 1 & - & 1 & - & - & - & 3 & - & - & - & 2 & 2 & - & - & - & - & - & 4 \\
\hline $\begin{array}{l}\text { Dentalina sp. } \\
\text { Egrerelly bradvi }\end{array}$ & - & 1 & $\bar{z}$ & 3 & - & 1 & ${ }_{5}$ & 1 & - & $z$ & - & - & - & - & - & - & 3 & - & - & - & - & - & - & - & - & - \\
\hline $\begin{array}{l}\text { Eggerella bradyi } \\
\text { Ehrenbergina serrata }\end{array}$ & -9 & ${ }_{-}^{4}$ & $=$ & 1 & ${ }^{3}$ & ${ }^{3}$ & -5 & 2 & $\overline{-}$ & - & - & - & 2 & 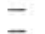 & $2^{2}$ & ${ }_{-}^{3}$ & $\bar{z}$ - & $\begin{array}{l}1 \\
3\end{array}$ & ${ }^{1}$ & $\bar{z}+x+1+$ & - & $\bar{z}+x+1+$ & $=$ & $=$ & - & $\bar{z}$ \\
\hline Ehrenbergina trigona & - & - & $=$ & 2 & 2 & ${ }_{3}$ & 5 & 6 & $\overline{-}$ & - & - & -1 & $z$ & $=$ & $\bar{z}$ & $\bar{z}$ & $=$ & - & $\bar{z}+2+x$ & $\bar{z}$ & - & - & - & - & & $=$ \\
\hline Epistominella exigua & 6 & 21 & - & - & 3 & 2 & 3 & 9 & 3 & - & 6 & 2 & $\bar{z}$ & 6 & 1 & . & 6 & 12 & 4 & 5 & 3 & 4 & 6 & 5 & 2 & - \\
\hline Eponides tumidulus & - & $\overrightarrow{-}$ & - & - & 1 & - & - & - & - & - & - & i & - & 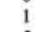 & - & - & 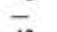 & - & 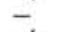 & - & - & - & - & - & & - \\
\hline Fissurina, Lagena, Oolina & 6 & 10 & 6 & 8 & 8 & 6 & 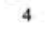 & 4 & 7 & 5 & 4 & 4 & 6 & 8 & 7 & 3 & 12 & 7 & 4 & 11 & 9 & 1 & 6 & 8 & 3 & 1 \\
\hline Francesita advena & - & $\bar{z}$ & 1 & - & - & - & - & - & - & - & - & - & - & - & - & - & - & - & - & - & - & - & - & - & - & - \\
\hline $\begin{array}{l}\text { Fursenkoina schreibersiana } \\
\text { Globocossidulina shoplobos }\end{array}$ & $2^{2}$ & $\bar{z}$ & -7 & -5 & -7 & -7 & - & - & 1 & -70 & - & - & - & $\overline{1 s}$ & - & - & - & $\overline{17}$ & $\overline{-}$ & -8 & - & - & 1 & 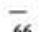 & $-\overline{2}$ & -8 \\
\hline $\begin{array}{l}\text { Globocassidulina subgl } \\
\text { Globulina, Guttulina }\end{array}$ & $\bar{z}$ & $=$ & - & -5 & ${ }_{-}^{7}$ & -7 & 7 & -9 & -6 & 20 & ${ }^{9}$ & 12 & II & 15 & $\frac{1}{2}$ & 7 & 4 & 17 & $=$ & 8 & -3 & $\begin{array}{l}4 \\
3\end{array}$ & -13 & $\begin{array}{r}66 \\
1\end{array}$ & 26 & 8 \\
\hline $\begin{array}{l}\text { Gyroidinoides spp. } \\
\text { Gyrom }\end{array}$ & 3 & 5 & 5 & 6 & $\overline{12}$ & 7 & $\overline{14}$ & 7 & 6 & 5 & -9 & $\bar{z}_{3}$ & ${ }_{8}$ & 6 & 9 & 8 & 9 & 7 & 7 & 10 & 5 & 14 & 5 & 11 & 7 & 8 \\
\hline reria & $i$ & $i$ & $\mathrm{i}$ & 0 & - & - & & - & & - & 1 & - & - & 0 & $y$ & & & & & & 2 & & $i$ & 2 & . & - \\
\hline muperata & . & - & 2 & 2 & - & 1 & & 1 & - & 1 & & 1 & 1 & 1 & i & - & - & & & - & & - & & - & & - \\
\hline & - & 1 & - & 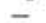 & - & i & & 1 & - & - & $=$ & - & i & 1 & 1 & 1 & 1 & & & - & 2 & 1 & & & 6 & - \\
\hline Meloni & 1 & - & - & 2 & - & - & 1 & - & 5 & 4 & - & 2 & $\mathrm{i}$ & - & i & & & & , & 6 & 9 & & & 12 & 10 & 2 \\
\hline des & - & 1 & 1 & - & 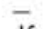 & - & - & - & - & - & - & - & - & - & - & - & - & - & & & - & & & 1 & & 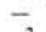 \\
\hline $\begin{array}{l}\text { Melonis sp. } \\
\text { Nodosaria Stilostomet }\end{array}$ & ${ }^{3}$ & $\bar{z}$ & 5 & ${ }_{9}^{2}$ & $\begin{array}{l}15 \\
12\end{array}$ & 1 & 14 & 6 & 3 & 10 & 11 & 7 & 14 & $?$ & 8 & - & $\overline{18}$ & 6 & ${ }_{34}^{5}$ & $7^{7}$ & 8 & ${ }_{22}^{13}$ & ${ }_{26}^{5}$ & 78 & $3^{3}$ & ${ }_{42}^{3}$ \\
\hline $\begin{array}{l}\text { Oodossara, } \\
\text { Oridorsalis umbonaturella }\end{array}$ & $\overline{14}$ & $\overline{14}$ & ${ }_{3}^{6}$ & $\frac{9}{4}$ & $\begin{array}{r}12 \\
1\end{array}$ & $\begin{array}{l}9 \\
4\end{array}$ & -8 & $\begin{array}{l}4 \\
4\end{array}$ & ${ }_{5}^{11}$ & $\begin{array}{r}14 \\
5\end{array}$ & 9 & $\begin{array}{r}10 \\
6\end{array}$ & $\begin{array}{r}16 \\
9\end{array}$ & ${ }_{15}^{11}$ & $\begin{array}{r}6 \\
12\end{array}$ & $\begin{array}{l}3 \\
3\end{array}$ & ${ }_{3}^{18}$ & 10 & $\begin{array}{r}34 \\
6\end{array}$ & $\frac{12}{7}$ & $\begin{array}{r}14 \\
4\end{array}$ & $\begin{array}{c}22 \\
1\end{array}$ & 7 & $\begin{array}{r}18 \\
4\end{array}$ & 11 & $\begin{aligned} 42 \\
5\end{aligned}$ \\
\hline Osan & - & - & - & - & - & 1 & 1 & - & - & - & 1 & - & - & - & - & - & - & - & - & - & - & - & - & - & - & - \\
\hline & - & 1 & 1 & - & - & 2 & - & 3 & - & - & 1 & - & 4 & - & 1 & 2 & & - & - & 1 & - & - & - & 12 & - & - \\
\hline & 2 & 2 & 4 & - & 1 & 9 & - & 7 & 4 & 1 & 3 & 8 & 5 & 3 & 7 & 5 & 13 & 8 & 6 & 12 & 8 & 3 & 1 & - & - & - \\
\hline Pleurosto & - & 1 & - & - & - & - & 4 & - & 6 & 5 & 4 & - & 1 & 2 & 2 & - & 1 & - & - & 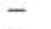 & 2 & 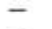 & 3 & 2 & 1 & t \\
\hline $\begin{array}{l}\text { Pullenia } \\
\text { Pullenia }\end{array}$ & 1 & 5 & - & - & 5 & 1 & - & - & 2 & 2 & $\bar{u}$ & 1 & 1 & 3 & 11 & 2 & 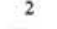 & 5 & 5 & - & 2 & - & - & 2 & 2 & 6 \\
\hline 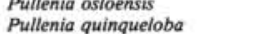 & $\tau_{1}$ & ${ }_{-}^{1}$ & $\begin{array}{l}2 \\
2\end{array}$ & $-{ }_{2}$ & $T_{4}$ & $\tau_{1}$ & $I$ & $\begin{array}{l}5 \\
2\end{array}$ & $\begin{array}{l}9 \\
1\end{array}$ & -8 & 11 & ${ }_{3}^{4}$ & $\begin{array}{l}8 \\
4\end{array}$ & $\bar{z}$ & $\bar{z}$ & $\bar{z}$ & ${ }_{3}$ & ${ }_{1}^{4}$ & -1 & -1 & ${ }_{3}^{2}$ & _ & $\overline{1}$ & $=$ & 2 & $\bar{t}_{1}$ \\
\hline Pull & 3 & 1 & 1 & 2 & i & - & & - & $f^{2}$ & 1 & 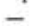 & - & 2 & - & - & - & i & - & 2 & 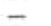 & - & 2 & - & - & - & - \\
\hline Pyr. & 3 & 6 & 2 & - & $i$ & 1 & & 1 & - & . & - & - & - & - & - & - & - & - & - & - & - & - & 3 & - & - & - \\
\hline & 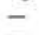 & 1 & - & 3 & - & & & - & - & - & - & - & - & - & - & - & - & - & - & - & - & - & - & $\neg$, & - & - \\
\hline$Q_{u}$ & - & - & - & - & - & - & & 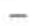 & - & - & - & - & - & - & 1 & - & - & - & 2 & - & 2 & 3 & - & $-{ }^{\prime}$ & - & - \\
\hline & - & - & - & 1 & 3 & 1 & 2 & - & 1 & - & - & 2 & - & - & - & - & - & - & - & 1 & - & - & - & - & - & 1 \\
\hline & 1 & 1 & 4 & 6 & , & 1 & 2 & - & 1 & 2 & - & 2 & - & - & 1 & - & - & 1 & 4 & 1 & 2 & 1 & - & - & - & - \\
\hline & - & - & - & & - & - & - & - & - & - & - & - & - & - & - & - & - & - & - & - & - & 9 & - & 4 & 2 & 7 \\
\hline & - & - & - & - & - & 1 & - & 1 & - & 1 & - & - & - & - & 2 & 2 & 1 & - & - & - & 2 & - & - & - & - & - \\
\hline & - & 1 & - & 1 & 3 & - & - & - & - & - & 1 & 1 & - & - & - & - & - & - & - & - & 5 & 1 & 3 & 2 & - & - \\
\hline bili & - & - & - & - & - & - & - & $=$ & - & - & - & - & - & 4 & 3 & 7 & - & - & - & - & - & - & - & - & $\bar{z}$ & 2 \\
\hline ia & 2 & 2 & $\bar{z}$ & - & $\bar{\tau}_{2}$ & $\bar{z}$ & - & $\bar{z}$ & $\bar{z}_{3}$ & - & $\bar{z}$ & - & $\overline{-}$ & $\bar{z}$ & - & $\bar{z}$ & - & & 7 & $\bar{z}$ & $\overline{-}$ & - & - & - & - & - \\
\hline & - & - & - & - & - & 1 & - & - & - & 2 & 3 & 2 & 6 & 5 & - & 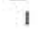 & . & - & 2 & 1 & 2 & - & - & - & - & - \\
\hline & 4 & . & & 16 & 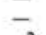 & 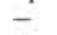 & & - & & & 2 & 1 & & - & - & & - & 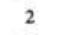 & & & & 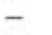 & & - & - & 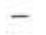 \\
\hline & - & - & - & 4 & 2 & 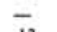 & - & 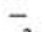 & - & - & 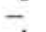 & . & - & - & & - & - & & & & - & - & & 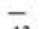 & - & \\
\hline Uniden & 9 & 1 & - & & 1 & 13 & 7 & 2 & 6 & 4 & 1 & 4 & 7 & 1 & 6 & 4 & 1 & 7 & 8 & 7 & 9 & 6 & 6 & 13 & 4 & 3 \\
\hline Total & 104 & 113 & 103 & 127 & 118 & 115 & 112 & 109 & 121 & 120 & 113 & 109 & 126 & 106 & 131 & 107 & 107 & 116 & 148 & 118 & 121 & 106 & 112 & 184 & 120 & 116 \\
\hline umber & 35 & 38 & 33 & 42 & 3 & 36 & 34 & 34 & 31 & 34 & 31 & 3 & 32 & 26 & 44 & 29 & 30 & 39 & 41 & 36 & 40 & 32 & 28 & 33 & 29 & 25 \\
\hline $\begin{array}{l}\text { Ratio planktonic to } \\
\text { benthic }(x: 1)\end{array}$ & 99 & 99 & 99 & 99 & 98 & 95 & 49 & 99 & 99 & 99 & 99 & 99 & 99 & 99 & 99 & 99 & 99 & 99 & 99 & 49 & 24 & 24 & 24 & 10 & 49 & 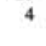 \\
\hline
\end{tabular}

Note: Dash $=$ absent in assemblage count.

igua, Globocassidulina subglobosa, Gyroidinoides spp., Melonis spp., Nodosaria/Stilostomella, Oridorsalis umbonatus, and $P$. wuellerstorfi. The diversity ranges from $\propto 10$ to 25 and the planktonic to benthic ratio is high except in the early Miocene where dissolution has taken place.

The $P$ wuellerstorfi fauna suggests depths $>1500$. Melonis pompilioides, which occurs at depths $>2000 \mathrm{~m}$, is present as rare specimens in only four samples. This suggests depths of $<2000 \mathrm{~m}$. Thus, it seems reasonable to interpret the depth of deposition from early Miocene to Recent at Site 555 as 1500 to 2000 m (cf. site depth of $1666 \mathrm{~m})$.

The early Miocene in Hole 553A lacks $P$. wuellerstor$f i$ itself, although in other respects a $P$. wuellerstorfi type assemblage is present. The rarity of $E$. exigua is indicative of depths $<2500 \mathrm{~m}$. Modern $B$. alazanensis and $O$. umbonatus have an upper depth limit of $1500 \mathrm{~m}$, so the interpreted depth range cannot be narrowed closer than 1500 to $2500 \mathrm{~m}$. The same applies to the early Miocene of Sample 554A-4-2, $16 \mathrm{~cm}$.

\section{Q-mode Varimax Factor Analysis}

All the Neogene assemblages from Holes 552A, 553, $553 \mathrm{~A}, 554,554 \mathrm{~A}$, and 555 have been subjected to a comparative analysis with the Q-mode varimax factor analysis of the recent samples (see contribution by J. F. Weston). The dominance of the varimax factors (i.e., factor score of 0.5 to 1.0 or -0.5 to -1.0 ) and the percentage of variance accounted for are listed in Table 8 .

The most complete stratigraphic record is that of Hole 552A (HPC). Factor 1 appears in the middle Miocene, continues through to the early Pliocene, and reappears in the Pleistocene. This same factor is recorded either frequently or intermittently in the early Miocene to late Pliocene and Pleistocene of the other sites. This factor 


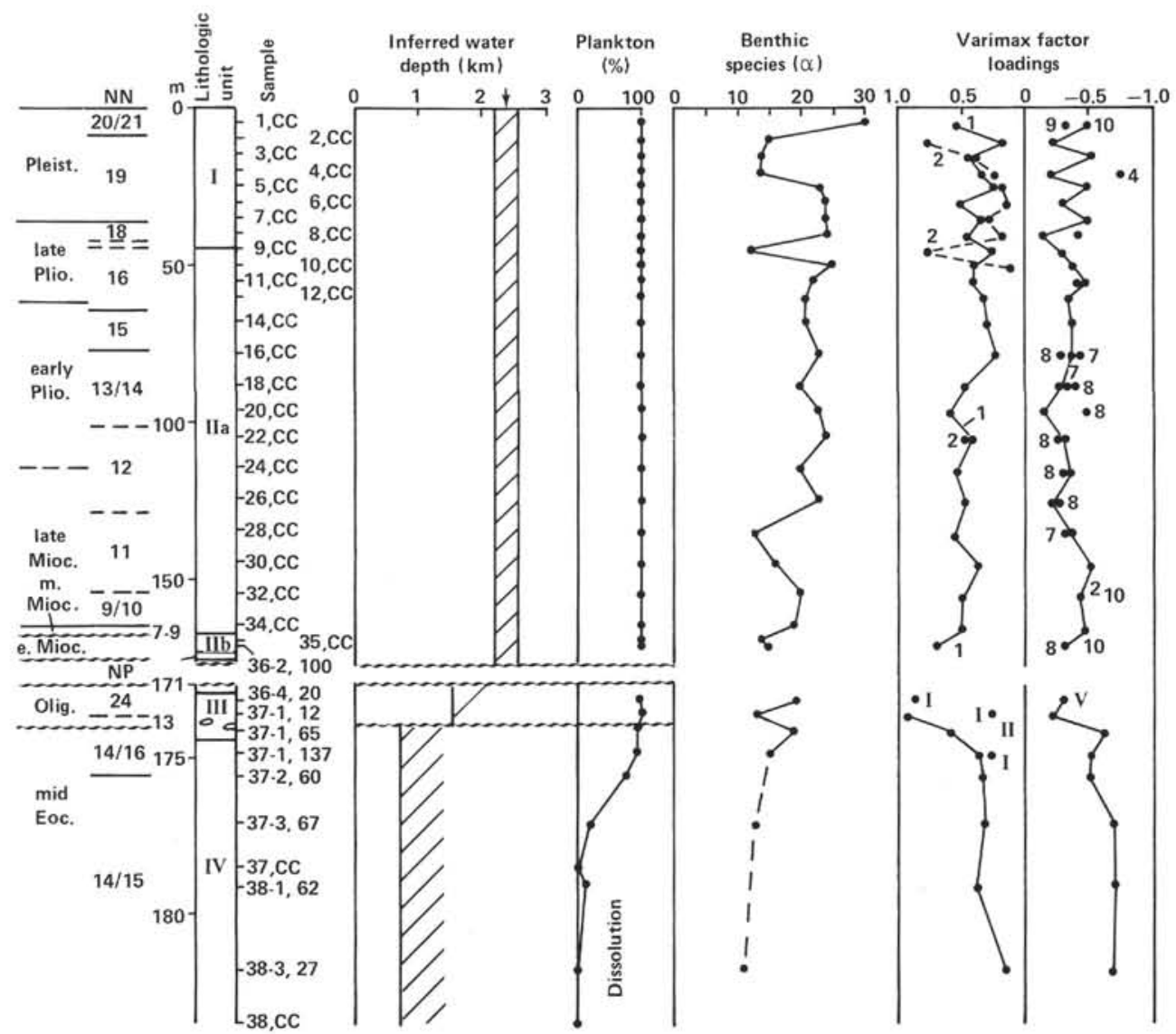

Figure 4. Hole 552A. Biostratigraphy based on nannozones from Backman (this volume), lithological units from site chapter (this volume), and inferred water depth at the time of deposition, determined from the benthic foraminifers. (Note expanded scale for the Paleogene. $\downarrow=$ present site depth.)

represents modern NADW assemblages, which at present occur to the south of this area (Fig. 2) and at greater depths $(>3500 \mathrm{~m})$.

In Hole $552 \mathrm{~A}$, factor 8 is subdominant from the middle Miocene to early Pliocene. At present this factor represents a mid-latitude assemblage occurring at a depth of 2000 to $2800 \mathrm{~m}$ (Hole 552A was drilled in a depth of $2301 \mathrm{~m})$. This factor is also recorded in the early Miocene of Site 555 (depth $1659 \mathrm{~m}$ ).

Factor 10, which at present is correlated with newly formed Norwegian Sea Overflow Water (Fig. 1), is occasionally dominant in the Miocene (Holes 554A and 555), late Pliocene (Hole 552A), and Pleistocene (Holes 552A and $553 \mathrm{~A})$.

Factors 2 and 4 are confined to the glacial late Pliocene and Pleistocene. Factor 2 is ill-defined in that it is based on the dominance of Cassidulina, but the species recorded in the Leg 81 samples $(C$. teretis) is not the same as those recorded in the Recent samples $(C$. carinata and $C$. obtusa). Factor 4, however, represents the modern assemblage found in the Rockall area, and is associated with Labrador Sea Water and NEADW.

It must be stressed that only $\sim 50 \%$ of the variance is accounted for by these factors in Neogene samples; therefore, the results must be interpreted with caution. Nevertheless, it seems evident that a northern source of bot- tom water was active from early Miocene times onwards. On the limited evidence available it appears that the water masses of the Miocene and early Pliocene were fairly stable, while those of the glacial late Pliocene and Pleistocene were more variable.

In conclusion, the stability of the faunas through the Neogene and the fact that the varimax factors based on modern assemblages can be derived from the Neogene assemblages suggests that changes in bottom water circulation have been of degree rather than of kind.

\section{PALEOGENE ASSEMBLAGES}

The assemblage counts are listed in Tables 9 to 12 and the more important general characters are shown in Figures 4 to 9 . In contrast to the Neogene assemblages, those of the Paleogene are much more variable, reflecting more diverse environments of deposition.

\section{Oligocene}

This is present in Holes $552 \mathrm{~A}, 553 \mathrm{~A}$, and $554 \mathrm{~A}$. The assemblages have moderate to high diversity $(\propto 13-24)$ and are dominated by Nodosaria/Stilostomella spp., Gyroidinoides spp., Globocassidulina subglobosa, and Osangularia spp. Other forms present include Oridorsalis ecuadorensis, and "Neogene" species such as Bulimina alazanensis, $O$. umbonatus, Cibicidoides $\mathrm{cf}$. C. kullen- 
BENTHIC FORAMINIFERS

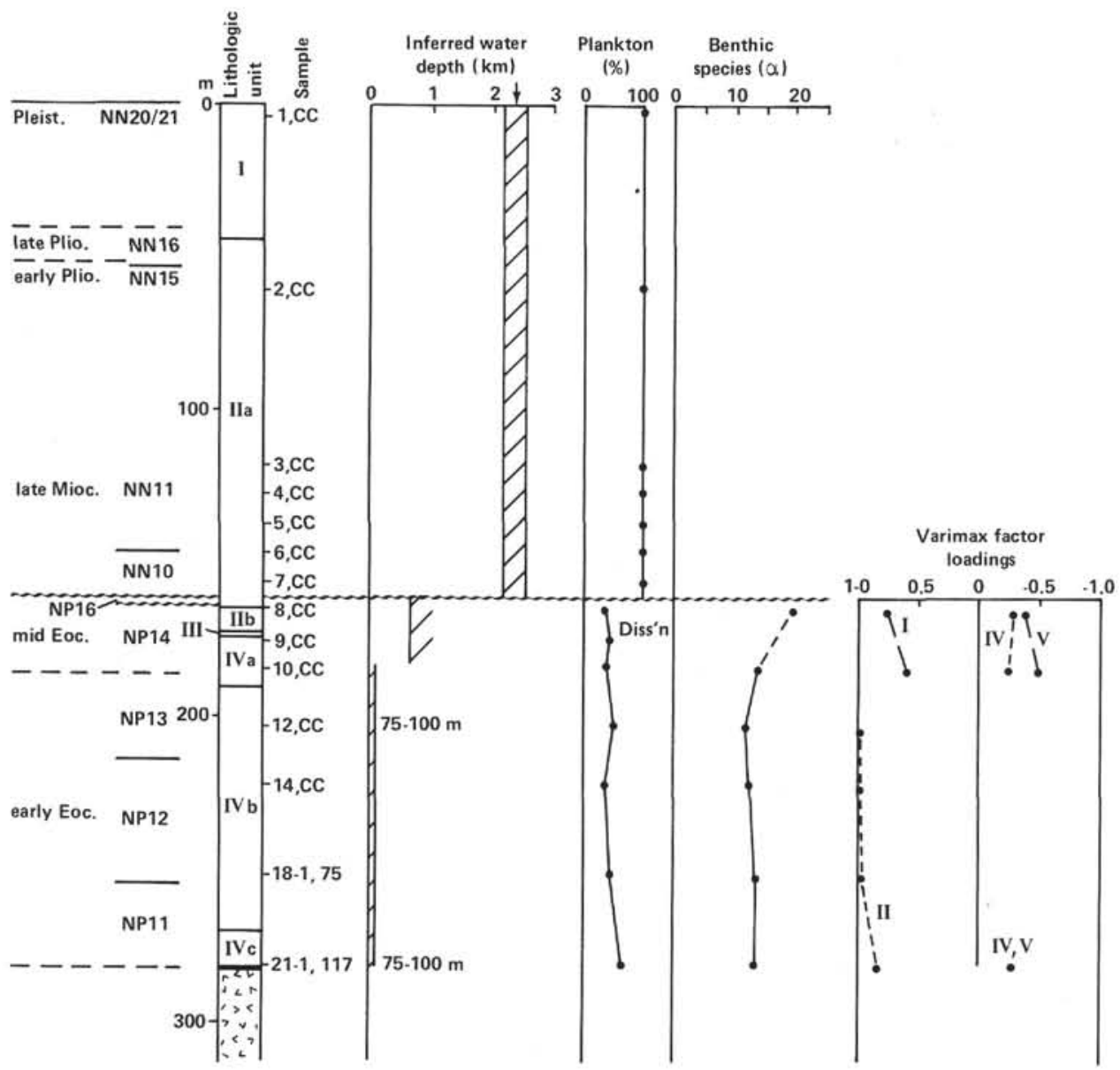

Figure 5. Hole 552. Biostratigraphy based on nannozones from Backman (this volume), lithological units from site chapter (this volume), and inferred water depth at the time of deposition, determined from the benthic foraminifers. $(\downarrow=$ present site depth. $)$

bergi, and Astrononion guadelupae. In Hole 554A, Spiroplectammina spectabilis and Nonion havanensis are additional common species.

Most of these assemblages are from condensed sequences that have been affected by dissolution. Preservation is commonly poor and the planktonic to benthic ratio is often low because of the preferential loss of planktonic forms.

\section{Late Eocene}

Absent from all holes except 554A, where the fauna is dominated by Nodosaria/Stilostomella spp., are Globocassidulina subglobosa and Oridorsalis ecuadorensis. The diversity is moderate $(\propto 9-13)$ and the planktonic to benthic ratio low as a result of dissolution.

\section{Middle Eocene}

In Holes 552, 552A, and 553A the dominant species are Alabamina wilcoxensis, Bulimina tuxpamensis, Nodosaria/Stilostomella spp., Cibicidoides spp., Gyroidinoides spp., Nuttallides spp., and Osangularia sp. Dissolution has affected these assemblages to a greater or lesser extent, some levels being almost barren (e.g., Hole 552: 9, CC and Hole 552A: 37-2, $137 \mathrm{~cm} ; 37, \mathrm{CC} ; 38-1$, $62 \mathrm{~cm}$; and $38, C C)$. With the exception of 553A-9-6,
$51 \mathrm{~cm}$ to $553 \mathrm{~A}-10-3,66 \mathrm{~cm}$, which have a planktonic to benthic ratio of $99: 1$, the remainder have low values, probably mainly because of the effects of dissolution.

\section{Early Eocene-Late Paleocene}

Assemblages of this age were recovered from all four sites. Eleven different assemblages are recognized according to the environment of deposition:

1. Osangularia spp. dominant with Nodosaria/Stilostomella spp. subsidiary, but affected by dissolution (555-27-2, $105 \mathrm{~cm}$ and $28-1,37 \mathrm{~cm})$.

2. Anomalinoides howelli dominant with various accompanying subsidiary species: Pulsiphonina prima and/ or A. nobilis (552-12,CC to $21-2,117 \mathrm{~cm}$; 555-31-1, $20 \mathrm{~cm}$ to 32,CC); Gavelinella semicribrata, Cibicidoides spp., Lenticulina spp., and Elphidium hiltermanni; (553A-11-2, $80 \mathrm{~cm}$ to 16,CC); Anomalinoides sp., E. hiltermanni, Nonion laeve, and Pararotalia curryi (553A18,CC); Protelphidium sp. (553A-19,CC).

3. Gyroidinoides spp. dominant, with subsidiary Anomalinoides acutus, Lenticulina spp., Nodosaria spp., Eponides spp., Cibicidoides alleni, Alabamina obtusa, and Praeglobobulimina ovata $(555-44, \mathrm{CC} ; 45, \mathrm{CC})$.

4. Nodosaria/Stilotomella spp., Lenticulina spp. dominant, with subsidiary Praeglobobulimina ovata, 


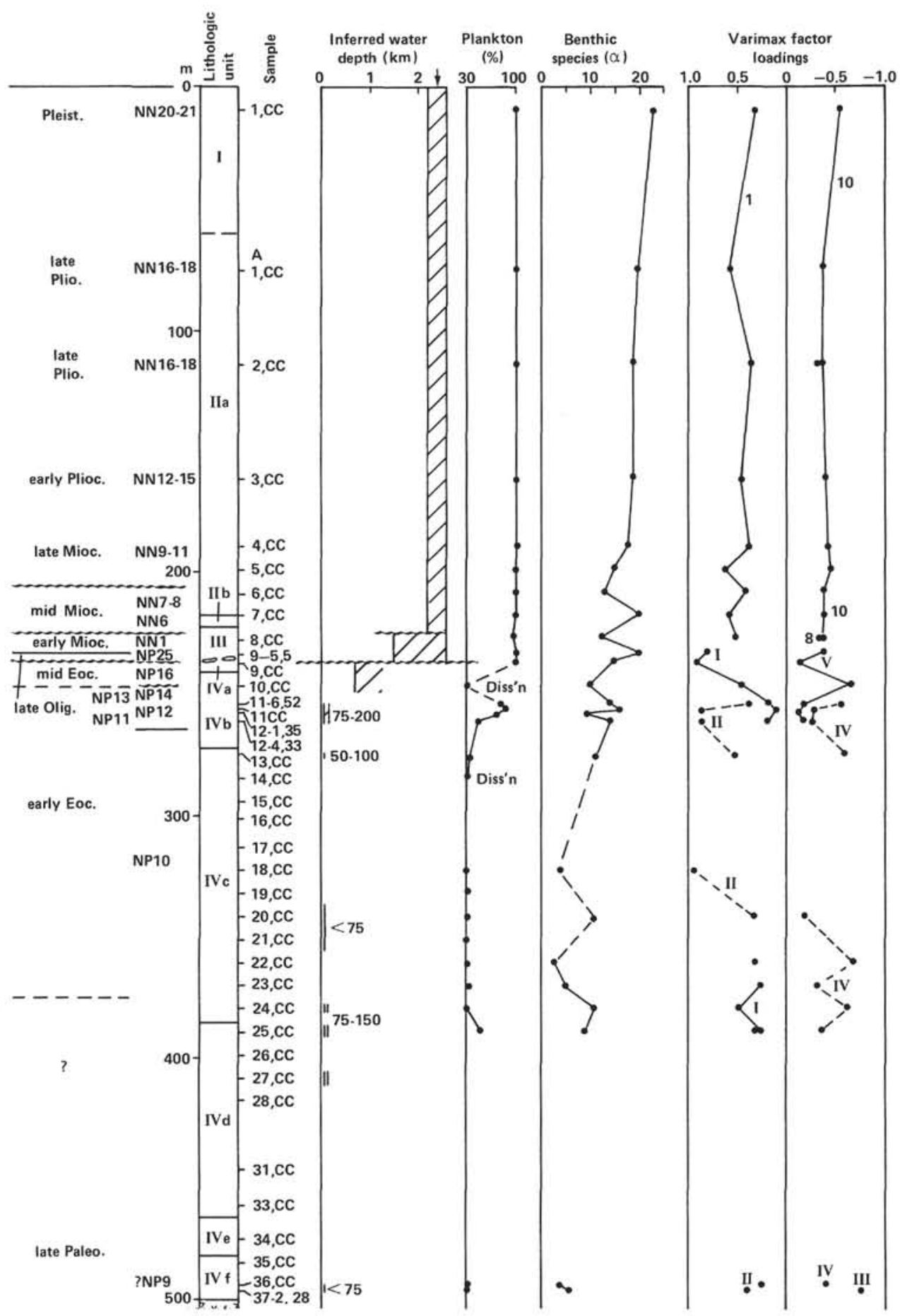

Figure 6. Holes 553, 553A. Biostratigraphy based on nannozones from Backman (this volume), lithological units from site chapter (this volume), and inferred water depth at the time of deposition, determined from the benthic foraminifers. $(\downarrow=$ present site depth.)

Anomalinoides acutus, Alabamina obtusa, Cibicidoides alleni, and Pullenia sp. (553A-20,CC; 21,CC; 23,CC to $37-2,28 \mathrm{~cm})$.

5. Lenticulina spp. dominant with subsidiary Alabamina obtusa and Anomalinoides nobilis (555-47,CC; 48, $\mathrm{CC})$.
6. Praeglobobulimina ovata dominant with subsidiary Anomalinoides acutus, A. nobilis, Cancris subconicus, and Nonionella sp. (555-54,CC to 61,CC).

7. Cibicides westi dominant $(555-67,4 \mathrm{~cm} ; 64 \mathrm{~cm})$.

8. Cribrostomoides $\mathrm{sp}$. dominant with some of the following species: Anomalinoides nobilis, Nodosaria 

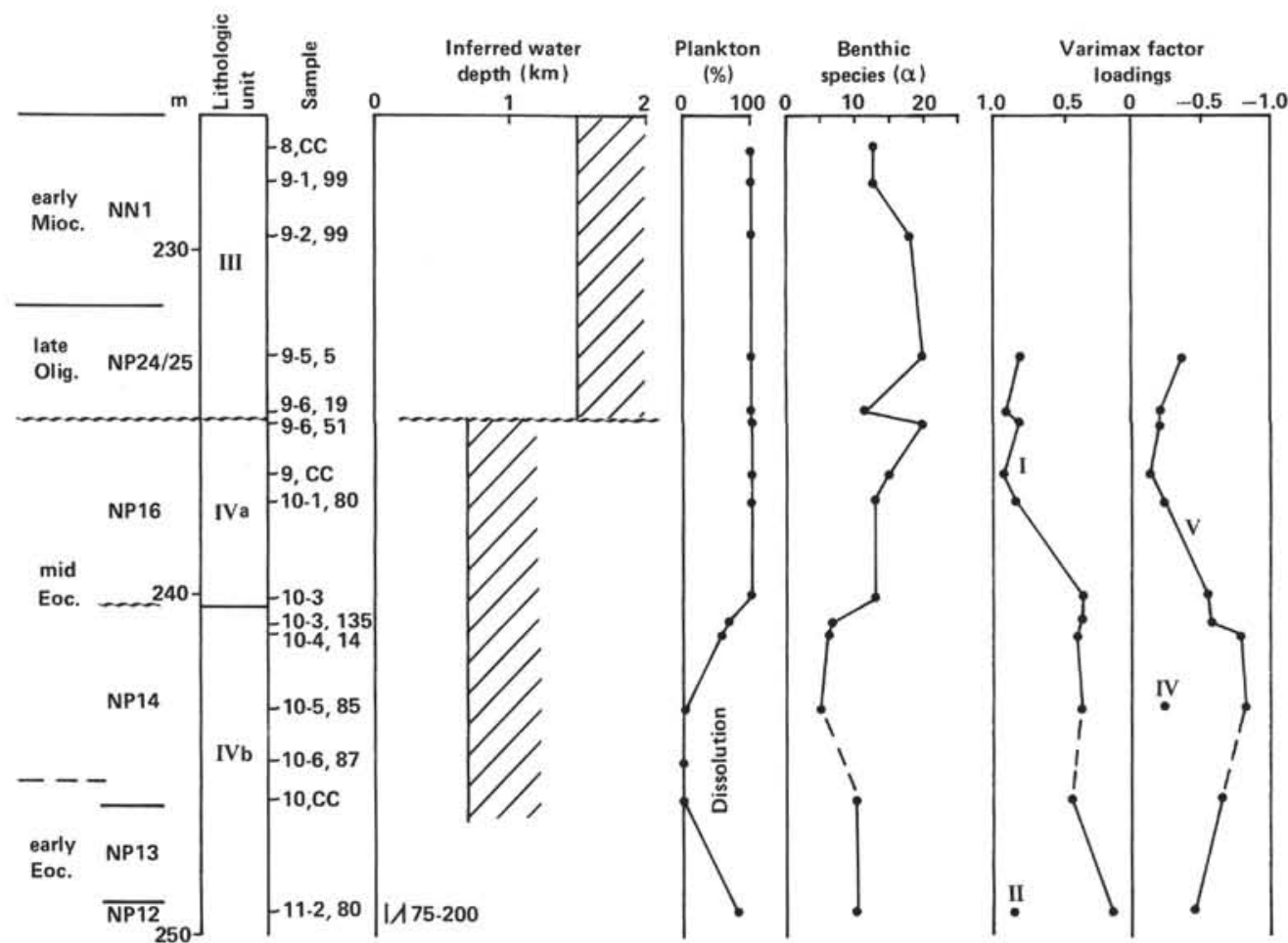

Figure 7. Hole 553A. Detail of condensed sequence shown in the center of Figure 6.

spp., Lenticulina spp., Praeglobobulimina ovata, and/ or Anomalinoides acutus (555-41,CC to 43,CC; 53,CC; 553A-22,CC).

9. Nonionella spp. dominant with subsidiary Cancris subconicus $(555-38, \mathrm{CC})$.

10. Protelphidium sp. dominant, either alone (555$52, \mathrm{CC}$ ) or with Cancris subconicus and Alabamina obtusa $(555-46, \mathrm{CC})$.

11. Trochammina sp. dominant with subsidiary $\mathrm{El}$ phidium hiltermanni and Anomalinoides acutus (553A$36, \mathrm{CC})$.

\section{Summary}

It is clear that with the strong environmental control on the distribution of species and assemblages, their use in biostratigraphy is limited. However, the following species which are confined to the late Paleocene to early Eocene in the Anglo-Franco-Belgian Basin (Jenkins and Murray, 1981) are also restricted to the same level at the four sites, with the two exceptions noted:

\section{Alabamina obtusa}

Bolivinopsis adamsi

Anomalinoides nobilis extend into middle Eocene Gaudryina hiltermanni in Hole 553A

Pulsiphonina prima, which is normally indicative of this same interval (although it extends into the middle Eocene in France; Jenkins and Murray, 1981), is confined to the late Paleocene to early Eocene at Sites 552 to 555 .

Nuttalides truempyi is confined to the Eocene.

\section{Environmental Interpretation}

The criteria for the interpretation of the Paleogene assemblages have already been discussed.
Brackish assemblages are restricted to the late Paleocene-early Eocene interval. An intertidal marsh is represented in 553A-36, CC by an assemblage of Trochammina sp. (48\%), Elphidium hiltermanni (17\%), and Anomalinoides acutus (13\%). The diversity is $\propto 4$, and no planktonic forms are present.

Protelphidium sp. makes up $97 \%$ of the assemblage in 555-52,CC. The diversity is $\propto<1$. This is interpreted as brackish intertidal with a salinity of 10 to $25 \%$. This sample contains much plant debris. Protelphidium sp. and Cancris subconicus dominate 555-46,CC, and this is thought to represent inner shelf to outer lagoon with salinities in the range 25 to $30 \%$.

Slightly brackish inner shelf ( $<75 \mathrm{~m}$ depth) environments are thought to be represented by the following: Nonionella sp. (78\%), C. subconicus $(19 \%)$, diversity $\propto 1$ assemblage of 555-38,CC; the Cribrostomoides sp. $(35 \%)$, Lenticulina spp. (20\%), Praeglobobulimina ovata $(16 \%)$, diversity $\propto 3$ assemblage of 553A-22,CC; and the $A$. howelli dominated assemblages of 553A-18,CC and 19,CC, which include some Elphidium hiltermanni, Pararotalia curryi, and Protelphidium sp.

Inner-shelf assemblages of normal salinity (depth $<75 \mathrm{~m}$ ) include those dominated by one or the other of the following species: $P$. ovata, Pulsiphonina prima, Epistominella vitrea, Cribrostomoides sp., A. acutus, Lenticulina spp., Eponides spp., or Cibicides westi. The diversity is commonly $\propto 5$ to 10 and planktonic foraminifers are either absent or rare $(<5 \%)$. Such assemblages are common in the early Eocene and late Paleocene (Hole 553A: 20,CC; 23,CC; 37-2, $28 \mathrm{~cm}$; and Hole 555: $33, \mathrm{CC} ; 41, \mathrm{CC}$ to $45, \mathrm{CC} ; 47, \mathrm{CC} ; 48, \mathrm{CC} ; 53, \mathrm{CC}$ to $67-4$, $64 \mathrm{~cm}$ ). At Site 555 this part of the succession contains oyster horizons (Hole 555: 33,CC; 48,CC; 49,CC) and 


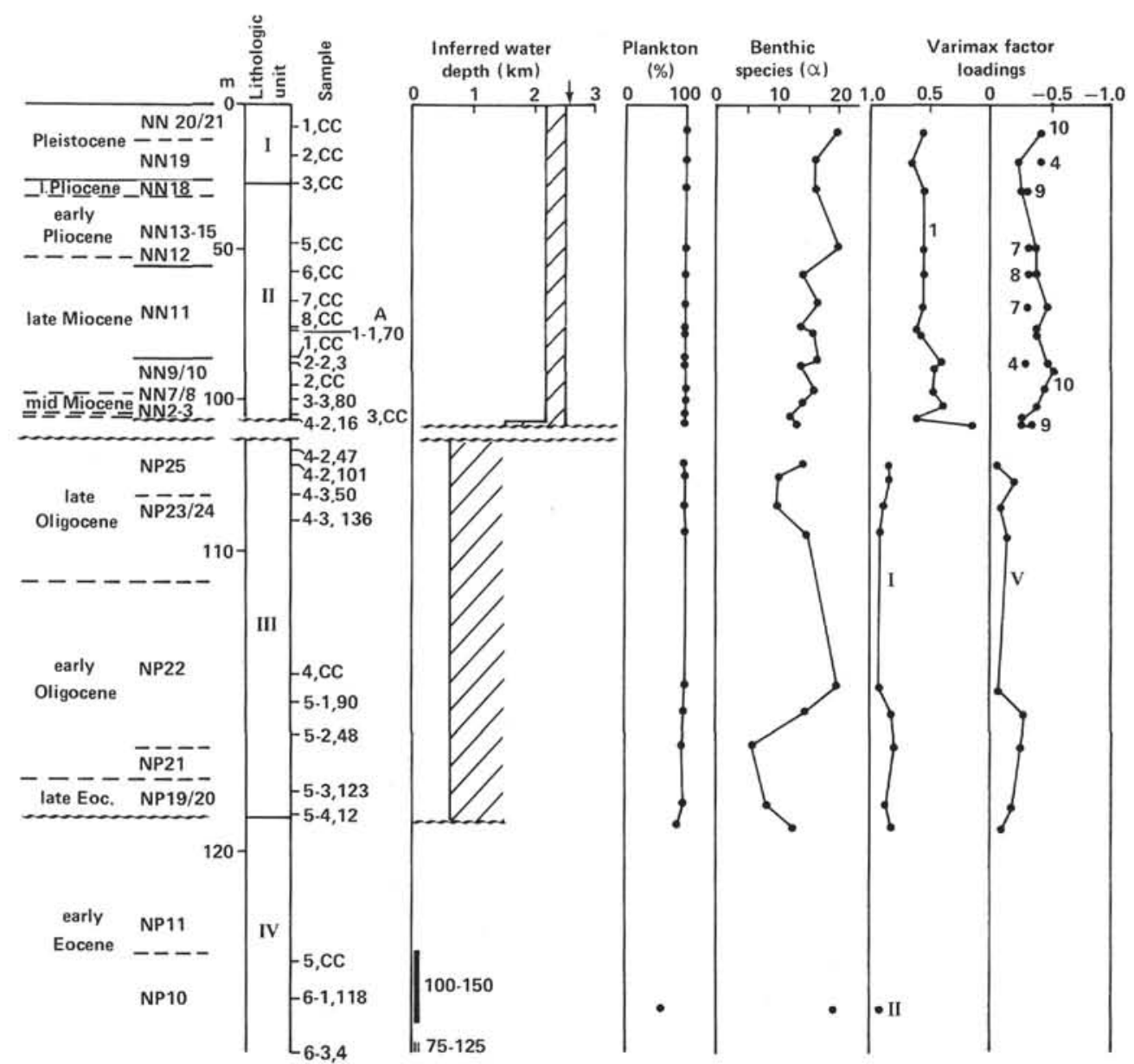

Figure 8. Holes 554, 554A. Biostratigraphy based on nannozones from Backman (this volume), lithological units from site chapter (this volume), and inferred water depth at the time of deposition determined from the benthic foraminifers. $(\downarrow=$ present site depth. Note the change of scale for the Paleogene.)

other bivalve mollusks. Sample 553A-13,CC with Lenticulina sp., Cibicides, and Bolivinopsis adamsi-a diversity of $\propto 11$, no planktonic foraminifers, and an associated fauna of bryozoa, molluskan shells bored by clionid sponges, and echinoid spines-is believed to have come from inner- to mid-shelf depths of 50-100 m.

Mid-shelf assemblages (75-150 m) are of two kinds: Those dominated by $A$. howelli, diversity $\propto 12-19$, $40-65 \%$ planktonic forms $(552-12, \mathrm{CC}$ to $21-2,117 \mathrm{~cm}$; 554A-6-1, $118 \mathrm{~cm}$ ) and those dominated by Cibicidoides alleni or Nodosaria spp., with Lenticulina spp., Alabamina obtusa, and $P$. ovata, diversity $\propto 9-11,0$ to $23 \%$ planktonic forms (553A-24,CC; 25,CC).

Mid- to outer-shelf assemblages (75-200 m) are composed of A. howelli, Gavelinella semicribrata, Gaudryina hiltermanni, and Lenticulina spp., with some $B$. adamsi and Cibicidoides sp. The diversity is $\propto 9-16$, and planktonic foraminifers make up 23 to $75 \%$ of the total (Samples 553A-11-2, $80 \mathrm{~cm}$ to $12-4,33 \mathrm{~cm}$ ).

Similar assemblages at Site $555(31-1,120 \mathrm{~cm} ; 31, \mathrm{CC}$; $32, \mathrm{CC})$, which are also dominated by $A$. howelli, have a diversity of $\propto 12-20$ and planktonic forms range from 26 to $72 \%$. Preservation is moderate.
Probable outer shelf assemblages $(150-200 \mathrm{~m})$ are those of 555-27-2, $105 \mathrm{~cm}$ and 555-28-1, $37 \mathrm{~cm}$. Both are poorly preserved through dissolution. The planktonic to benthic ratios are 17:83 and 7:93. The dominant forms are Osangularia spp. and diversity is $\propto 5$ to 9 .

Assemblages thought to represent bathyal depths $>700 \mathrm{~m}$ are of early Eocene (553A-10,CC), middle Eocene $(553 \mathrm{~A}-9-6,51 \mathrm{~cm}$ to $10-3,135 \mathrm{~cm} ; 552-8, \mathrm{CC}$ to $10, \mathrm{CC})$, late Eocene $(554 \mathrm{~A}-5-3,123 \mathrm{~cm} ; 5-4,12 \mathrm{~cm})$, and Oligocene (554A-4-2, $47 \mathrm{~cm}$ to $5-2,48 \mathrm{~cm}$ ) age. All are characterized by a dominance of Nodosaria/Stilostomella spp., commonly with subsidiary Oridorsalis ecuadorensis and sometimes with Cibicidoides spp., Gyroidinoides spp., Globocassidulina subglobosa, Alabamina wilcoxensis, Nonion havanensis, and Bulimina tuxpamensis. The diversity is $\propto 14-20$ in those samples not affected by dissolution, but drops as low as $\propto 4$ where dissolution is marked. Likewise, the planktonic to benthic ratio is high $(98: 2$ or $99: 1)$ except where dissolution has taken place, when values may fall to zero.

In Hole 552A, the middle Eocene assemblages interpreted as bathyal $>700 \mathrm{~m}$ have $A$. wilcoxensis or $B$. tuxpamensis as the dominant species together with some 
Nuttallides truempyi and Osangularia spp. Some dissolution has taken place as shown by the diversity value of $\propto 11-15$ and the planktonic proportion of $0-90 \%$.

The Paleogene assemblages believed to represent the deepest water $(>1500 \mathrm{~m})$ are of Oligocene age. The dominant species are Nodosaria/Stilostomella spp. and Gyroidinoides spp., with some Osangularia spp., Cibicidoides cf. kullenbergi, O. ecuadorensis, and rare B. alazanensis. The diversity is $\propto 12-24$ and the planktonic to benthic ratio is high, $94: 60$ to $99: 1$ (552A-36-4, $20 \mathrm{~cm}$; $552 \mathrm{~A}-37-1,12 \mathrm{~cm}$; 553A-9-5, $5 \mathrm{~cm}$; 553A-9-6, $19 \mathrm{~cm}$ ).

\section{Q-mode Varimax Factor Analysis}

The 71 samples on which counts of $>100$ individuals were made have been treated together for Q-mode vari$\max$ factor analysis. The taxa were organized into 34 groups as listed in Table 13. Five factors were used to describe the variability. The taxonomic composition of these is listed in Table 14, with the varimax factor scores expressed as percent for each taxon.

The factors have been plotted on Figures 4 to 9, and the distribution of each factor with respect to age and interpreted depth of deposition is listed in Table 15. A very clear picture is evident. Marginal marine environments are represented by factors II and IV in part, but in some cases none of the factors had any significant loading. This reflects the unusual and varied composition of these assemblages. The inner shelf is dominated by factors II, III, and IV, the mid shelf by II, III, IV, and V, and the outer shelf by II and V. These environments are found only in strata of late Paleocene to early Eocene age. The slope is characterized by factors I and V. Thus, as might be expected, there is a great deal of agreement between the subdivision into environments based on a paleoecological analysis and the subdivision using Q-mode factor analysis. However, it should be noted that the latter does not take into account such aspects as diversity, planktonic to benthic ratio, or the effects of dissolution.

\section{FAUNAL COMPARISONS}

Comparisons of faunas within the North Atlantic must take into account both the stratigraphic age of the assemblages and the environment of deposition. Even when these conditions are satisfied problems arise because of the lack of taxonomic agreement between workers in North America and Europe.

Figure 11 summarizes the depths of deposition interpreted for each of the numbered sites. No abundance data for the Neogene assemblages of Sites 111, 116, 117, 336, 352 and the Paleogene assemblages of Sites 336 and 352 are available. Also van Hinte (1976) did not give interpreted depths of deposition. For Site 336 he compared the fauna with the Barton Clay of England and this has been interpreted by Murray and Wright (1974) as of generally inner- to middle-shelf depths, i.e., $100 \mathrm{~m}$. The Oligocene fauna of Site 352 was said to indicate shallow water (van Hinte, 1976, p. 45) but was also said to be similar to that at Site 116, which Berggren and Aubert (1976b) interpret as bathyal, $800-1000 \mathrm{~m}$. Van
Hinte regarded the differences in the Oligocene faunas of Sites 336 and 352 as indicative of the existence of a faunal barrier between the Atlantic and the NorwegianGreenland Sea, i.e., the Iceland-Faeroes Ridge. This is not a reasonable conclusion on the evidence, for if Site 352 is bathyal and Site 336 shallow shelf, then two quite dissimilar environments are being compared.

\section{Quaternary and Neogene}

On the basis of depth and location the sites fall into three groups: Iceland-Faeroes Ridge, shallow (Sites 336, 352); Rockall Plateau (Sites 555, 116, 117) and Orphan Knoll (Site 111), shallow; Rockall Plateau margins, deeper (Sites 403-406, 552-554). The faunas from these regions have been summarized in Table 16 .

In the "glacial" late Pliocene and Pleistocene the faunas of Rockall margins are similar to those of Rockall Plateau and Orphan Knoll except that some of the deeper water indicators such as Planulina wuellerstorfi and Epistominella exigua are less common at the shallower sites. However, from the limited data in van Hinte (1976) it appears that the fauna of the Iceland-Faeroes Ridge is much less diverse and somewhat different in composition. This reflects the modest depth and the different water masses influencing the area. The glacial succession is characterized by the occurrence of Cassidulina teretis (recorded as C. laevigata at Sites $403-406$, by Murray, 1979). However, this species was not recorded from Orphan Knoll by Berggren (1972). In the Norwegian-Greenland Sea the incoming of $C$. teretis (Islandiella teretis of van Hinte, 1976) is at $\sim 5$ m.y. ago in the late Pliocene, long before the onset of glaciation in the North Atlantic (Schrader et al., 1976). Thus its first appearance throughout the area is clearly diachronous.

The Pliocene and late Miocene assemblages are treated together because they are so similar. Comparison of the lists in Table 16 shows that the deeper waters of Rockall margins and the shallow waters of Rockall Plateau and Orphan Knoll have many elements in common but certain species are rare or absent from one or the other as a result of differing depth and water mass preferences, e.g., Epistominella exigua, Planulina wuellerstorfi.

In the middle Miocene there are again many elements in common, with Brizalina subaenariensis being much more abundant on Rockall Plateau than in the deeper water of its margins.

In the early Miocene the depth differences between the areas are modest (Fig. 11). The faunas are essentially the same. Siphonina tenuicarinata and Oridorsalis ecuadorensis are both common elements. $P$. renzi and Anomalina alazanensis are recorded only from Orphan Knoll.

\section{Paleogene}

In the Oligocene all the sites with records were bathyal except for Site 336 (Fig. 11). The bathyal assemblages are composed of Cibicidoides or Heterolepa spp., Nodosaria/Stilostomella spp., Globocassidulina subglobo$s a$, and Oridorsalis umbonatus or O. ecuadorensis. The 


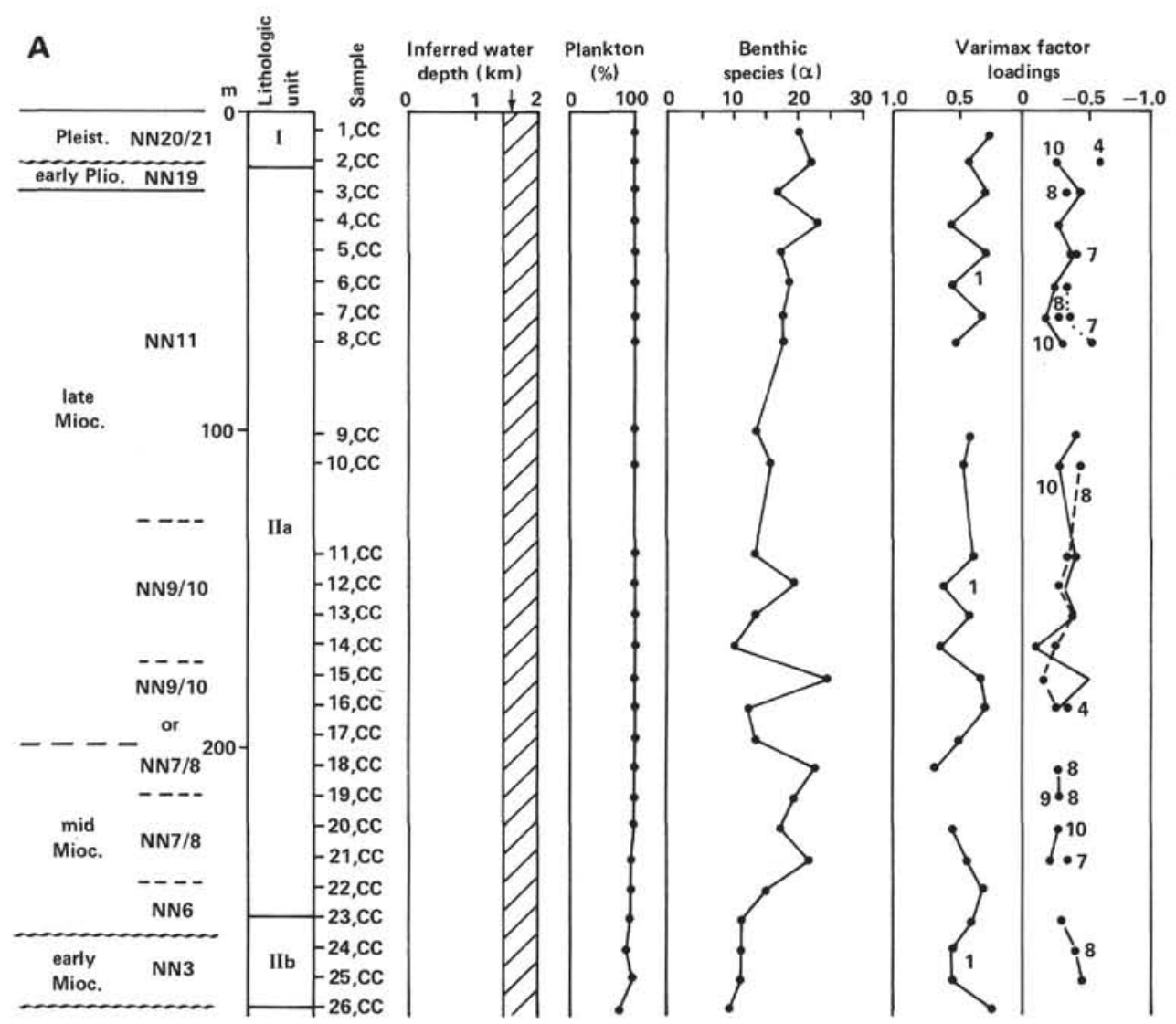

Figure 9. Hole 555. A. Neogene. B. Paleogene. Biostratigraphy based on nannozones from Backman (this volume), lithological units from site chapter (this volume), and inferred water depth at the time of deposition determined from the benthic foraminifers. $(\downarrow=$ present site depth.)

assemblage from Site 352 , as already stated, is of this type. By contrast, that at Site 336 is dominated by $A n$ gulogerina gracilis tenuistriata and Silicosigmoilina sp.

The late Eocene assemblages of Sites 554 and 406 are essentially of Oligocene bathyal type but those of Sites 111 and 116/117 differ: Site 111, Cibicidoides spp., Siphonina wilcoxensis, Osangularia mexicana; Sites 116, 117, Nuttallides truempyi, Anomalinoides grosserugosus. At Site 336 two distinct assemblages were recognized: Cibicides cf. C. tenellus; and Spiroplectammina spectabilis and Lenticulina cultrata. Both of these are shelf assemblages.

The middle Eocene deeper water assemblages comprise Cibicidoides spp., Nodosaria/Stilostomella spp., Bulimina spp., O. ecuadorensis, and Osangularia sp., sometimes with Alabamina wilcoxensis and Globocassidulina subglobosa. At Site 403 there is an epibathyal assemblage of $O$. ecuadorensis and Gyroidinoides spp. $(\sim 150 \mathrm{~m})$ and an outer shelf assemblage of Anomalinoides howelli, Cibicidoides spp., and Osangularia expansa.

In the early Eocene, the only bathyal assemblages are from Sites 111: Cibicidoides spp., Bulimina spp., Gaudryina hiltermanni, Nuttallides truempyi, Stilostomella spp., and Oridorsalis ecuadorensis; and Sites 405, 406: Nodosaria/Stilostomella spp., Bulimina trigonalis and Nonion havanensis (given as $N$. cf. olssoni in Murray, 1979). At Site 117, Berggren (1974) interpreted an as- semblage of Gavelinella grosserugosa, Bulimina brevispira, Gaudryina hiltermanni, Oridorsalis ecuadorensis, Osangularis pteromophalia, and Vaginulina decorata as 250-600 m (Fig. 11), but this could well be somewhat shallower (150-250 m).

The early Eocene to late Paleocene shelf assemblages of all sites are very similar and have already been described.

In conclusion, it is evident that notwithstanding differences in taxonomy, there is a great deal of similarity in the assemblages of comparable age and environment of deposition from the whole area.

\section{DISCUSSION}

These results have implications for the interpretation of the geological evolution of the area, especially the rate of subsidence, the existence of faunal barriers, and the development of water masses.

\section{Subsidence}

The pattern of subsidence is discussed in more detail in Roberts et al., this volume. Suffice it to point out that, as already established on Leg 48 (Murray, 1979), the benthic foraminifers show that subsidence and sedimentation more or less kept pace with one another in the late Paleocene and early Eocene; all the environments are of shallow water type, and some of the barren intervals may indicate emergence and even periods of eustatic 


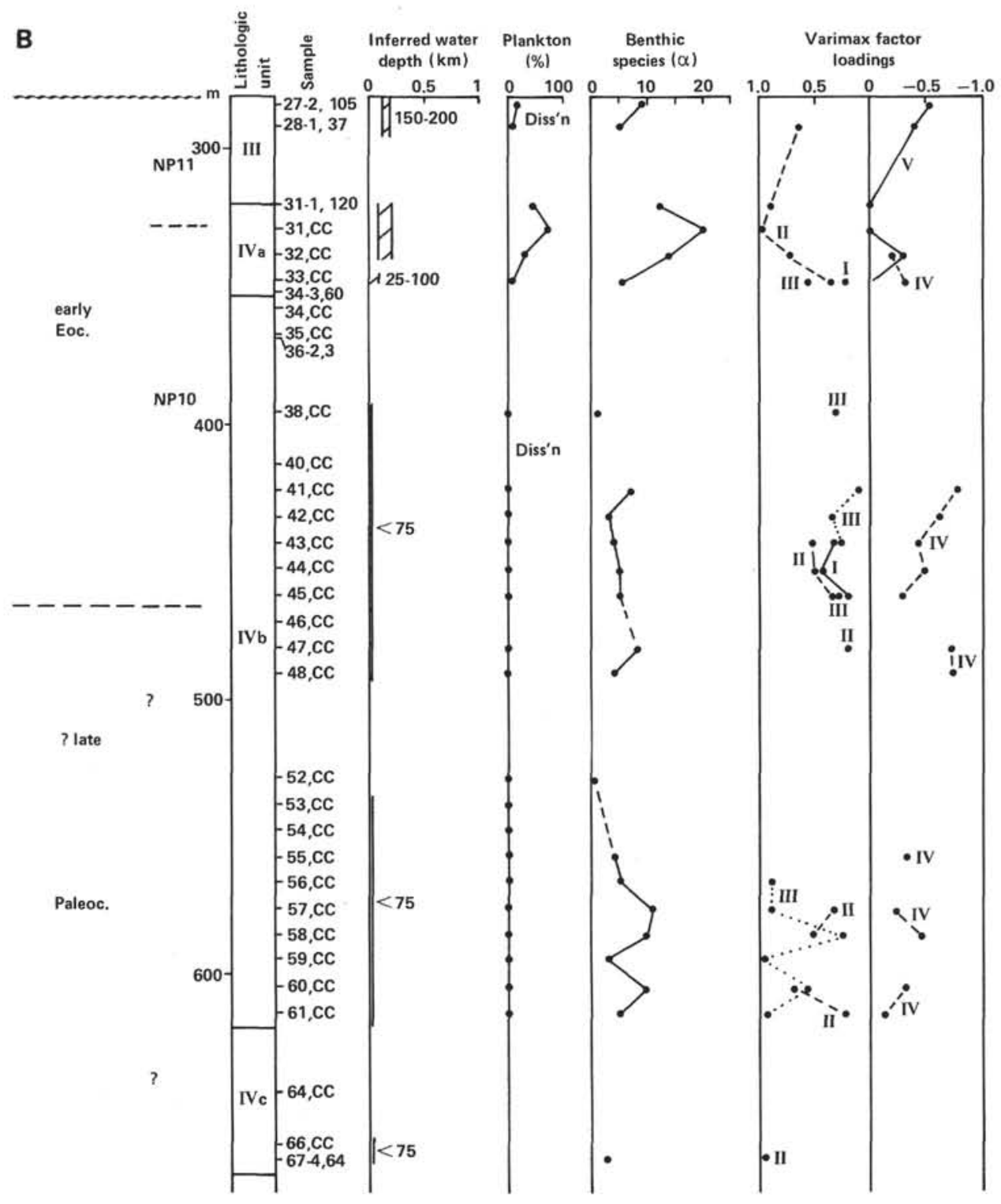

Figure 9. (Continued).

sea-level change (Vail, et al., 1977). However, from then on, the supply of sediment was inadequate to compensate for the rate of subsidence, so the water got progressively deeper. The incomplete late Eocene to early Miocene record, the effects of dissolution, and uncertainty about the ecological requirements of some Paleogene taxa, all make it difficult to assign precise depths to environments of this period. However, all sites seem to have subsided to around their present depths by early to middle Miocene times.

\section{Faunal Barriers}

Barriers to the migration of benthic faunas may be of many kinds, ranging from unsuitable substrates through salinity and temperature gradients to emergent land masses. In the context of the North Atlantic, most discussion has centered on the existence of the Greenland-Iceland-
Faeroes-Scotland Ridge as a land barrier at least during Paleogene times (see Bott, et al., in press).

It is beyond the scope of this chapter to consider all the arguments, geophysical, tectonic and paleontologi$\mathrm{cal}$, for and against this hypothesis. It is, however, appropriate to consider the evidence from the benthic foraminifers. The key to this is a comparison of the Paleogene fauna of the North Atlantic with those from the Norwegian-Greenland Sea. Apart from Sites 336 and 352 on the ridge itself, two other sites drilled on Leg 38 yielded good early Eocene faunas. At Site 338, the early Eocene is characterized by Lenticulina spp., Dentalina spp., Nodosaria latejugata, and Stilostomella spinulosa together with Spiroplectammina spectabilis, Textularia plummerae, Bulimina cacumenata, Turrilina brevispira, Chilostomelloides eocenica, Quadrimorphina paleocemica, Melonis affinis, Pullenia quinqueloba, Anomalinoi- 


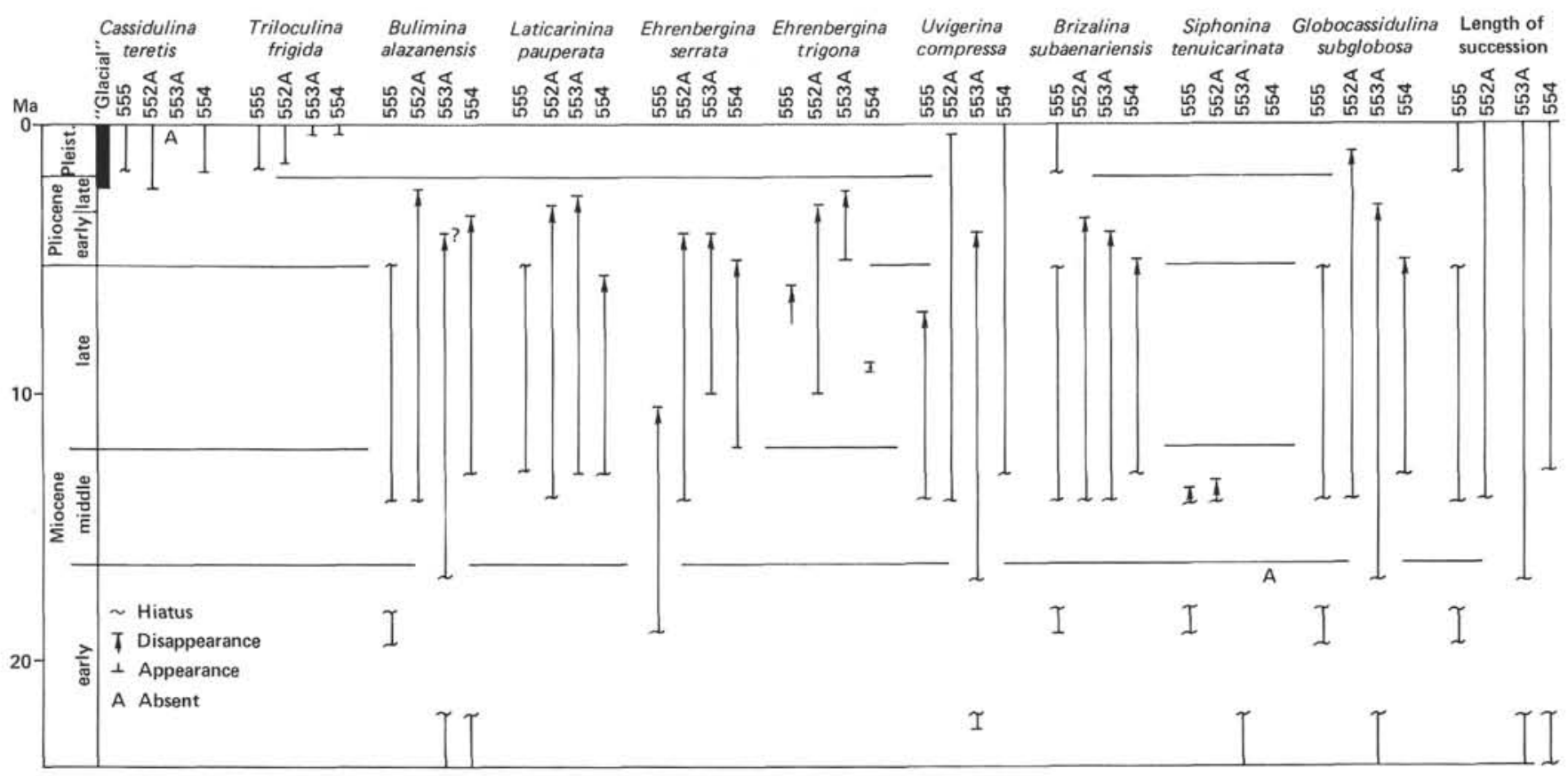

Figure 10. Neogene species showing restricted stratigraphic distributions.

Table 8. Q-mode varimax factors and percentage of variance accounted for, Neogene.

\begin{tabular}{|c|c|c|c|c|c|c|c|c|}
\hline \multirow[t]{2}{*}{ Hole } & \multicolumn{2}{|c|}{$552 \mathrm{~A}$} & \multicolumn{2}{|c|}{$553 \mathrm{~A}$} & \multicolumn{2}{|c|}{554} & \multicolumn{2}{|c|}{555} \\
\hline & Factor & $\begin{array}{c}\text { Variance } \\
(\%)\end{array}$ & Factor & $\begin{array}{c}\text { Variance } \\
(\%)\end{array}$ & Factor & $\begin{array}{c}\text { Variance } \\
(\%)\end{array}$ & Factor & $\begin{array}{c}\text { Variance } \\
(\%)\end{array}$ \\
\hline Pleistocene & $1 / 2 / 10(4)$ & $59-75$ & 10 & 48 & 1 & $59-62$ & (4) & $14-43$ \\
\hline Glacial Pliocene & $2 / 4$ & $59-60$ & & & & & & \\
\hline late Pliocene & (10) & $55-59$ & 1 & 50 & & & & \\
\hline early Pliocene & $1(8)$ & $54-62$ & - & 53 & 1 & 59 & & \\
\hline late Miocene & $1(8)$ & $54-57$ & 1 & $50-53$ & $1(10)$ & $56-60$ & (1) (7) & $43-56$ \\
\hline middle Miocene & $1(8)$ & 56 & 1 & 51 & 1 & 57 & (1) (10) & $49-55$ \\
\hline early Miocene & & & (1) & $50-52$ & - & 54 & $1 / 8$ & $47-51$ \\
\hline
\end{tabular}

Note: Only those factors with values 0.5 to 1.0 or -0.5 to 1.0 are listed; numbered factors in brackets only rarely reach these scores.

des anomalinoides, and Cancris subconicus. Some of these species occur at Site 343 (van Hinte, 1976 in Talwani, Udintsev, et al., 1976). These forms are also present in the North Atlantic (Berggren, 1974; Berggren and Aubert, 1976a, b; Murray, 1979; this chapter).

It could therefore be argued that on the evidence of the benthic foraminifers there is no reason to suspect the existence of a land barrier between the Norwegian-Greenland Sea and the North Atlantic. But if such a barrier existed, could the faunas on the two sides be so similar? The answer to this depends on alternative migration routes. The shallower water faunas of the North Atlantic and the Norwegian-Greenland Sea resemble those of the North Sea Basin, which certainly had connections with the North Atlantic through the English Channel from time to time during the Paleogene. The North Sea may also have been connected with Tethys through Europe. However, the faunas of the English Channel, especially its western part, are not at all like those of the North Sea, the Norwegian-Greenland Sea, or the Rockall area (Murray and Wright, 1974). It therefore seems more likely that the North Atlantic and the Norwegian-
Greenland Sea were in communication, even if only through a continuation of Rockall Trough into the Faeroes-Shetland Basin. Furthermore, such a connection need not have been deep.

\section{Water Masses}

Our understanding of the development of water masses is built up on evidence of the isotopic record, sediment type, and distribution, existence of hiatuses, as well as the distribution of the fauna. The distinction between modern oceanic water masses is based on small differences in temperature, salinity, density, etc., and this is reflected in subtle differences in faunal composition. At the present state of knowledge, it is not possible to detect such small changes in the Paleogene record and, indeed, many would regard it as presumptuous to recognize it in the Neogene and Quaternary records.

Using evidence from deep-sea benthic ostracods, Benson (1975) suggested that cold bottom water first appeared in the oceans at around the Eocene/Oligocene boundary. From oxygen isotopic evidence, Kennett and Shackleton (1976) inferred the existence of a two-layer 
Table 9. Benthic foraminiferal census data, Holes 552, 552A, Paleogene.

\begin{tabular}{|c|c|c|c|c|c|c|c|c|c|c|c|c|c|c|c|}
\hline \multirow[b]{3}{*}{ Species } & \multirow{2}{*}{\multicolumn{2}{|c|}{$\begin{array}{l}\text { m. Eoc. } \\
\text { Hole } 552\end{array}$}} & \multicolumn{4}{|c|}{ early Eocene } & \multicolumn{2}{|c|}{ Oligo. } & \multicolumn{7}{|c|}{ middle Eocene } \\
\hline & & & \multicolumn{13}{|c|}{ Hole 552A } \\
\hline & U. & $\begin{array}{l}U \\
0 \\
0\end{array}$ & $\begin{array}{l}\text { U } \\
\text { ב }\end{array}$ & $\begin{array}{l}\text { U } \\
\dot{\Xi}\end{array}$ & $\begin{array}{l}\approx \\
\stackrel{\dot{\infty}}{a}\end{array}$ & $\begin{array}{l}\Xi \\
\stackrel{i}{N}\end{array}$ & $\begin{array}{l}\text { ¿ } \\
\text { जु } \\
\text { d. }\end{array}$ & $\begin{array}{l}\simeq \\
\frac{1}{2}\end{array}$ & $\begin{array}{l}\tilde{\sigma} \\
\frac{\bar{c}}{m}\end{array}$ & $\begin{array}{l}\stackrel{5}{\leftrightarrows} \\
\frac{1}{5}\end{array}$ & $\begin{array}{l}8 \\
\text { i } \\
\frac{1}{m}\end{array}$ & $\begin{array}{l}5 \\
\hat{p} \\
\vec{m}\end{array}$ & $\begin{array}{l}\text { ర్ర } \\
\overrightarrow{1}\end{array}$ & $\begin{array}{l}\vec{N} \\
\vec{j} \\
\dot{m}\end{array}$ & $\begin{array}{l}\text { U. } \\
\text { d }\end{array}$ \\
\hline Alabamina obtusa & - & - & 1 & 1 & 5 & 8 & - & - & - & - & - & - & - & - & - \\
\hline Alabamina wilcoxensis & - & 18 & 3 & - & - & - & 1 & - & 10 & 1 & - & 16 & 8 & 40 & 1 \\
\hline Alabamina sp. & 3 & - & - & - & - & - & - & - & - & - & - & - & - & - & - \\
\hline Anomalinoides howelli & - & - & 76 & 36 & 45 & 34 & - & - & - & 5 & 1 & - & - & - & - \\
\hline Anomalinoides nobilis & - & - & - & - & - & 18 & - & - & - & - & - & - & - & - & - \\
\hline Anomalinoides sp. & - & - & - & - & 1 & - & - & - & 1 & - & - & - & - & - & - \\
\hline Astrononion guadelupae & - & - & - & - & - & - & 3 & - & - & - & - & - & - & - & - \\
\hline Bolivinopsis adamsi & - & - & - & - & - & 1 & - & - & - & - & - & - & - & - & - \\
\hline Brizalina sp. & 2 & 2 & 1 & 2 & 3 & - & - & 1 & 1 & - & - & - & - & - & - \\
\hline Bulimina alazanensis & - & - & - & - & - & - & 3 & - & 2 & 1 & - & - & - & - & - \\
\hline Bulimina praeinflata & - & - & - & - & - & 2 & - & - & - & - & 1 & - & 1 & - & - \\
\hline Bulimina tuxpamensis & 8 & - & - & - & - & 17 & - & - & - & - & - & 18 & 12 & 10 & - \\
\hline Bulimina sp. & 1 & 1 & - & - & - & - & 1 & 1 & - & - & - & - & - & - & - \\
\hline Cancris subconicus & - & - & 1 & - & - & - & - & - & - & - & - & - & - & - & 3 \\
\hline Cibicides/Cibicidina & 2 & 4 & 2 & 20 & 3 & 8 & 2 & 7 & 4 & - & - & 2 & - & 1 & - \\
\hline Cibicidoides alleni & - & - & - & - & - & 1 & - & - & - & - & - & - & - & - & - \\
\hline Cibicidoides cf. C. bradyi & - & - & - & - & - & - & 3 & - & - & - & - & - & - & - & - \\
\hline Cibicidoides cf. C. kullenbergi & - & - & - & - & - & - & 1 & 16 & - & - & - & - & - & - & - \\
\hline Cibicidoides sp. & 19 & 2 & - & 2 & 9 & 2 & 3 & - & 5 & 10 & 2 & 1 & 3 & 8 & - \\
\hline Dentalina sp. & 6 & - & - & - & 2 & - & 1 & - & - & - & 1 & - & - & - & - \\
\hline Elphidium hiltermanni & - & - & 1 & 1 & - & - & - & - & - & - & - & - & - & - & - \\
\hline Epistominella vitrea & - & - & - & - & 1 & - & - & - & - & - & - & - & - & - & - \\
\hline Eponides spp. & 2 & 1 & 2 & - & 1 & 4 & - & - & - & - & 1 & 6 & 1 & 1 & 2 \\
\hline Fisurina, Lagena, Oolina & 1 & - & 6 & 1 & 1 & 1 & 1 & 1 & 1 & - & - & 1 & - & 2 & - \\
\hline Gaudryina hiltermanni & - & - & - & - & - & 2 & - & - & - & - & - & - & - & - & - \\
\hline Gavellinella semicribrata & 2 & - & - & - & - & 2 & - & - & 1 & 4 & - & - & 1 & 1 & - \\
\hline Globocassidulina subglobosa & 2 & 1 & 5 & 2 & - & 4 & 28 & 3 & 1 & 5 & 6 & 11 & - & - & - \\
\hline Globulina, Guttulina & - & - & 2 & - & 1 & - & 1 & 3 & 1 & - & - & - & - & - & - \\
\hline Gyroidinoides spp. & 5 & 9 & 5 & 2 & 4 & 1 & 21 & 10 & 7 & 8 & 3 & 8 & 3 & 1 & - \\
\hline Lenticulina spp. & 9 & 6 & 1 & 1 & 1 & 8 & 1 & 6 & 10 & 4 & - & 1 & 1 & 11 & 2 \\
\hline Melonis sp. & 1 & 1 & - & - & - & - & - & 2 & - & 4 & 3 & 2 & 1 & - & - \\
\hline Nodosaria, Stilostomella & 21 & 14 & 2 & 2 & - & 5 & 28 & 19 & 15 & 3 & 1 & 7 & 9 & 9 & - \\
\hline Nonion havanensis & 6 & 6 & - & - & 2 & 2 & - & - & 7 & - & 2 & 10 & - & - & - \\
\hline Nonion laeve & - & - & - & - & 2 & - & - & - & - & - & - & - & - & - & - \\
\hline Nuttalides truempyi & - & - & - & - & - & - & - & - & 2 & 21 & 4 & 1 & - & - & - \\
\hline Oridorsalis ecuadorensis & 3 & 11 & 1 & - & - & - & 5 & - & 7 & 6 & - & 2 & 1 & - & - \\
\hline Oridorsalis umbonatus & - & - & - & - & - & - & 1 & 5 & - & - & - & - & - & - & - \\
\hline Osangularia expansa & - & - & 1 & 2 & 7 & 11 & - & - & - & - & - & - & - & - & - \\
\hline Osangularia rugosa & - & - & - & - & - & - & 3 & 1 & - & - & - & - & - & 1 & - \\
\hline Osangularia sp. & - & - & - & - & - & - & 14 & 1 & 14 & 17 & 8 & 1 & 3 & - & - \\
\hline Pararotalia curryi & - & - & - & - & 1 & - & - & - & - & - & - & - & - & - & - \\
\hline Pleurostomella sp. & 3 & 3 & - & - & 1 & - & 4 & 4 & 2 & 1 & 1 & 1 & 2 & 2 & 1 \\
\hline Protelphidium sp. & - & - & - & - & 1 & 1 & - & - & - & - & - & - & - & - & - \\
\hline Pullenia osloensis & - & - & - & - & - & - & 2 & - & - & - & - & - & - & - & - \\
\hline Pullenia quinqueloba & 2 & 3 & - & 1 & 1 & - & 1 & 1 & - & 2 & 1 & - & 2 & 2 & - \\
\hline Pulsiphonina prima & - & - & - & 4 & 12 & 6 & - & - & - & - & - & - & - & - & - \\
\hline Quinqueloculina sp. & - & - & 1 & - & - & - & - & - & - & - & - & - & - & - & - \\
\hline Siphonina lamarckana & - & 7 & - & - & - & - & - & - & - & - & 1 & 1 & - & 4 & - \\
\hline Spiroplectammina spectabilis & - & - & 1 & - & 2 & 4 & 1 & 4 & 9 & 1 & - & - & - & - & - \\
\hline Trifarina cuneata & 1 & - & - & - & - & - & - & - & 2 & 1 & - & - & - & - & - \\
\hline Turrilina brevispira & - & - & 2 & 3 & - & - & - & - & - & - & - & - & - & - & - \\
\hline Uvigerina abbreviata & 7 & 6 & - & - & - & 2 & - & 1 & - & 1 & 4 & - & - & - & - \\
\hline Unidentified & 25 & 10 & 12 & 18 & 10 & 6 & 22 & 11 & 18 & 20 & 9 & 13 & 5 & 11 & - \\
\hline Total & 134 & 105 & 126 & 98 & 116 & 150 & 151 & 100 & 120 & 115 & 49 & 102 & 53 & 104 & 9 \\
\hline Number of species & 39 & 30 & 29 & 28 & 32 & 34 & 40 & 28 & 36 & 32 & 24 & 28 & 17 & 26 & 5 \\
\hline $\begin{array}{l}\text { Ratio planktonic to } \\
\text { benthic }(x: 1)\end{array}$ & 0.6 & 0.6 & 1.1 & 0.6 & 0.8 & 1.9 & 16 & 99 & 10 & 11 & 2.7 & 0.2 & 0.09 & 0 & 0 \\
\hline Barren $9, \mathrm{CC}, 13, \mathrm{CC}$ & & & & & & & & & & & & & & & \\
\hline
\end{tabular}

Note: Dash $=$ absent in assemblage count.

ocean, with the lower layer (psychrosphere) having a temperature of less than $10^{\circ} \mathrm{C}$, at the Eocene/Oligocene boundary. This stratification was attributed to the production of extensive sea ice around Antarctica which, in turn, promoted cold bottom-water formation and more vigorous bottom circulation. The effect of these changes is evident in changes in sediment distribution in the oceans at this period (see, for example, Moore, et al., 1978). Recently, Corliss (1981) has reviewed the effect of this cooling on benthic foraminifers. His data for Site 277 in 
Table 10. Benthic foraminiferal census data, Hole 553A, Paleogene.

\begin{tabular}{|c|c|c|c|c|c|c|c|c|c|c|c|c|c|c|c|c|c|c|c|c|c|c|c|c|c|c|c|c|c|}
\hline \multirow[b]{2}{*}{ Species } & \multicolumn{2}{|c|}{ Oligo. } & \multicolumn{8}{|c|}{ middle Eocene } & \multicolumn{14}{|c|}{ carly Eocene } & \multicolumn{5}{|c|}{ late Paleocene } \\
\hline & $\dot{\alpha}$ & 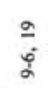 & 离 & $\underset{\mathrm{U}}{\mathrm{U}}$ & $\begin{array}{l}\Phi \\
\stackrel{+}{\dot{t}}\end{array}$ & $\begin{array}{l}8 \\
\text { శ్ }\end{array}$ & $\frac{\cong}{\stackrel{3}{6}}$ & 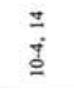 & 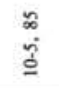 & $\begin{array}{l}0 \\
\dot{0} \\
\stackrel{\phi}{0}\end{array}$ & $\stackrel{8}{\square}$ & $\begin{array}{l}\stackrel{8}{\infty} \\
\stackrel{\Xi}{=}\end{array}$ & $\begin{array}{l}\tilde{N} \\
\stackrel{\varphi}{=}\end{array}$ & 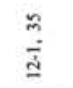 & $\begin{array}{l}\frac{m}{d} \\
\stackrel{+}{\beth}\end{array}$ & $\stackrel{\text { g }}{=}$ & 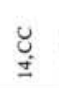 & $\stackrel{\underline{u}}{\underline{\Delta}}$ & 莒 & $\underline{\underline{u}}$ & ¿ั & $\frac{8}{i}$ & ت্ & $\underset{i}{\Delta}$ & 雚 & $\underset{\breve{d}}{\breve{d}}$ & 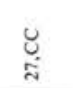 & 岕 & $\begin{array}{l}\text { क्ष } \\
\text { స్ }\end{array}$ \\
\hline $\begin{array}{l}\text { amina obtusa } \\
\text { amina wilcoxensis }\end{array}$ & $\bar{z}+$ & $\bar{z}+$ & $=$ & $=$ & $\bar{z}$ & $\overline{24}$ & $=$ & $\overline{10}$ & $\bar{z}$ & $\overline{13}$ & $-{ }_{5}$ & 1 & $\bar{z}$ & 4 & $\bar{z}$ & $2^{2}$ & $=$ & $=$ & $=$ & $\bar{z}$ & $\bar{z}$ & 2 & 1 & 2 & -8 & 18 & ${ }^{3}$ & $=$ & $z$ \\
\hline $\begin{array}{l}\text { bamina sp. } \\
\text { malinoides a }\end{array}$ & -5 & $\Xi$ & $\bar{z}$ & $\bar{z}$ & $\bar{z}$ & $\frac{-}{-}$ & $\bar{z}$ & $=$ & 12 & $=$ & $\bar{z}$ & -6 & $\bar{z}$ & $\overline{=}$ & $\overline{1}$ & $\bar{z}$ & $\bar{z}$ & $\bar{z}$ & $\bar{z}$ & $\bar{z}$ & $\overline{8}$ & $\overline{4}$ & $\frac{\overline{1}}{16}$ & $\frac{\overline{5}}{5}$ & $\overline{2}$ & $\frac{1}{2}$ & $\overline{1}$ & $\overline{22}$ & $\bar{z}$ \\
\hline $\begin{array}{l}\text { Inomalinoides howelli } \\
\text { inomalianodes nobilis }\end{array}$ & $\bar{z}+x$ & $=$ & $-^{3}$ & $=$ & $2^{2}$ & 1 & $=$ & $\bar{z}+x$ & $\overline{2}$ & $\bar{z}$ & $\bar{z}$ & 24 & ${ }_{5}^{3}$ & $\begin{array}{l}34 \\
2\end{array}$ & $\begin{array}{c}27 \\
2\end{array}$ & 3 & ${ }_{3}^{2}$ & $\bar{z}$ & 23 & 20 & $\frac{-}{2}$ & $\frac{4}{2}$ & $\frac{1}{2}$ & - & $-\frac{2}{4}$ & $\begin{array}{l}2 \\
5\end{array}$ & 2 & 2 & ${ }_{12}^{27}$ \\
\hline $\begin{array}{l}\text { les sp. } \\
\text { guadup }\end{array}$ & $-\tau_{4}>$ & $-{ }_{8}$ & $\bar{z}+$ & $\bar{z}+$ & $\bar{z}$ & $\bar{z}$ & $\bar{z}$ & $\bar{z}$ & $=$ & 2 & 3 & ${ }_{2}^{2}$ & - & - & - & $=$ & - & & 14 & $\overline{-}$ & - & - & - & $=$ & $i$ & $i$ & - & $=$ & - \\
\hline $\begin{array}{l}\text { giaded } \\
\text { adam }\end{array}$ & & & $z$ & $z$ & $\Xi$ & $z$ & $\bar{z}$ & $=$ & $\beth$ & $\bar{z}+$ & $\overline{-}+$ & - & $\overline{2}$ & $\overline{5}$ & $\overline{1}$ & $\overline{11}$ & 1 & $\overline{2}$ & - & & $\overline{1}$ & - & $\bar{z}$ & $\overline{7}$ & $\overline{-}_{4}$ & 2 & $\overline{-}$ & $\bar{z}$ & $\bar{z}+x+1$ \\
\hline limina alazzanensis & 2 & 1 & - & - & - & -2 & $\bar{z}$ & $\bar{z}$ & $\bar{z}$ & $\bar{z}$ & - & 6 & 1 & 2 & $\overline{-}$ & $\overline{-}$ & - & - & - & - & 2 & - & - & 2 & - & i & - & 2 & 2 \\
\hline $\begin{array}{l}\text { mina praeinflatata } \\
\text { mina }\end{array}$ & - & - & -2 & - & - & - & $=$ & $\overline{1}$ & $=$ & - & 2 & 1 & $\overline{1}$ & $=$ & $\bar{z}$ & $=$ & $=$ & $\overline{-}$ & $=$ & $\bar{z}$ & $\bar{z}$ & $\bar{z}$ & $\overline{-}$ & $\overline{-}$ & $=$ & $=$ & $\bar{z}$ & $=$ & $\bar{z}$ \\
\hline $\begin{array}{l}\text { ofanilis } \\
\text { pamensis }\end{array}$ & $\overline{-}$ & $\bar{z}$ & $\bar{z}$ & $=$ & $4^{4}$ & $2^{2}$ & $\overline{26}$ & $\overline{19}$ & $\overline{9}$ & $\stackrel{1}{-}$ & $\overline{7}$ & $\bar{z}$ & ${ }_{5}^{2}$ & $\bar{z}+x$ & $\overline{8}$ & $z$ & 1 & $\bar{z}$ & $\bar{z}$ & $\bar{z}$ & $\underline{-6}$ & $\bar{z}+$ & $\bar{z}$ & $\frac{7}{-}$ & $=$ & $\bar{z}$ & $=$ & $=$ & $\bar{z}$ \\
\hline $\begin{array}{l}\text { limina sp. } \\
\text { curis subec }\end{array}$ & $=$ & 1 & ${ }^{4}$ & 1 & $\bar{z}+$ & $\bar{z}$ & $=$ & $\bar{z}$ & $=$ & $\bar{z}$ & $\bar{z}$ & $\bar{z}$ & $=$ & - & - & - & - & - & - & - & - & - & - & - & - & 2 & - & - & $\bar{n}$ \\
\hline 15 & $\overline{-}$ & $=$ & $\bar{z}+$ & $=$ & $\bar{z}+$ & $=$ & $\overline{-}$ & $\overline{-}$ & $=$ & $=$ & $=$ & $\overline{-}$ & $\bar{s}$ & - & $\overline{7}$ & $\Xi$ & - & $z$ & 2 & & - & - & - & - & & 2 & $\overline{-}$ & - & 34 \\
\hline dina & - & - & - & - & - & - & $\bar{z}$ & $\overline{-}$ & $=$ & - & - & - & 2 & $\bar{z}$ & $-\frac{7}{3}$ & 17 & - & - & - & - & 6 & - & $\bar{z}$ & $\overline{3}$ & $\overline{1}$ & $\overline{4}$ & - & $=$ & - \\
\hline idoides of. c., br & 3 & $\overline{-}$ & $\overline{-}$ & 4 & - & $\bar{z}$ & $\overline{-}$ & $\bar{z}$ & $\bar{z}$ & $=$ & $\overline{-}$ & $\bar{z}$ & $\overline{-}$ & $\bar{z}$ & - & $-^{2}$ & $\overline{-}$ & $\overline{-}$ & $\bar{z}$ & $=$ & $=$ & $\bar{z}$ & $=$ & $\frac{3}{-}$ & 16 & 47 & 1 & $\bar{z}+$ & $\bar{z}$ \\
\hline 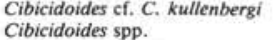 & 15 & $\bar{z}$ & $\overline{12}$ & $\begin{array}{l}6 \\
1\end{array}$ & $\begin{array}{c}18 \\
1\end{array}$ & $\overline{7}$ & $\overline{6}$ & $\overline{12}$ & $\overline{12}$ & $\bar{s}$ & $\overline{23}$ & $\overline{7}$ & $\overline{13}$ & $\overline{11}$ & $\overline{10}$ & $\bar{z}$ & $z$ & $\bar{z}$ & $\bar{z}$ & $\bar{z}$ & $\overline{-}$ & $=$ & $\bar{z}+$ & $\overline{-}$ & - & $\overline{10}$ & $\bar{z}$ & $\bar{z}+$ & $\bar{z}$ \\
\hline tomoides sp. & - & $\bar{z}+$ & $\bar{z}$ & $\bar{z}$ & $\bar{z}+$ & $\bar{z}$ & $\overline{-}$ & $\bar{z}$ & $\bar{z}$ & $\bar{z}$ & - & -78 & - & $\overline{-}$ & - & - & 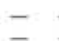 & - & 1 & & & - & 49 & - & - & - & - & - & - \\
\hline & 2 & $\overline{-}$ & $\bar{z}$ & $=$ & $=$ & 1 & $\bar{z}$ & $\bar{z}$ & $=$ & $=$ & - & $\frac{18}{-}$ & 1 & $\bar{z}$ & $\overline{-}$ & 1 & $\bar{z}$. & $\bar{z}$ & $\overline{-}$ & - & $\overline{-}$ & $\overline{-}$ & $\overline{-}$ & $\bar{z}$ & $\bar{z}+$ & - & 3 & - & $\bar{z}$ \\
\hline $\begin{array}{l}\text { tidum hillermanni } \\
\text { tominella vitrea }\end{array}$ & $\bar{z}$ & $\bar{z}$ & $\bar{z}$ & - & $\bar{z}$ & $\bar{z}$ & - & $\bar{z}$ & $\bar{z}+$ & $\bar{z}$ & & -5 & 14 & - & $\bar{z}$ & $\tau_{1}$ & $\overline{1}$ & & s. & & $\overline{37}$ & & $=$ & 10 & - & - & $\overline{-}$ & 29 & 3 \\
\hline . & - & - & - & - & - & 11 & 10 & - & - & - & - & 2 & - & - & - & $=$ & - & - & - & - & 等 & & - & & & & $\overline{-}$ & - & - \\
\hline $\begin{array}{l}\text { nidides spp. } \\
\text { urina, Lagena, Oolina }\end{array}$ & - & $\overline{1}$ & $\tau_{1}$ & $\bar{z}$ & $\bar{z}$ & - & $\overline{-}$ & $\bar{z}$ & $\bar{z}$ & $\bar{z}$ & $\bar{z}$ & - & $\overline{1}$ & $\bar{z}$ & $\overline{-}$ & 1 & $\bar{z}$ & $\bar{z}+$ & - & - & $\overline{-}$ & 1 & $\bar{z}$ & $\bar{z}$ & 1 & ${ }_{1}^{6}$ & ${ }^{4}$ & $-{ }^{3}$ & -5 \\
\hline Lus elongatum & - & $\bar{z}+$ & $\bar{z}$ & $\bar{z}+$ & $\bar{z}+$ & $\bar{z}$ & -3 & $\pi$ & $\overline{6}$ & $\bar{z}$ & -5 & $\overline{-}$ & $\dot{-}$ & $\overline{13}$ & $-\overline{2}$ & $=$ & $=$ & $\bar{z}+x \cdot+a$ & 2 & $\bar{z}$ & $\overline{-}$ & - & - & - & - & - & - & - & \\
\hline 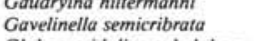 & $\bar{z}$ & $\bar{z}$ & $\overline{-}$ & $\bar{z}$ & 1 & $\bar{z}+x+x+$ & $\frac{3}{-}$ & ${ }_{2}^{\pi}$ & $\frac{6}{-}$ & $\bar{z}$ & -5 & $-\tau_{1}$ & $\overline{13}$ & $\frac{13}{8}$ & $\frac{2}{3}$ & $\Xi$ & $\bar{z}$ & $\bar{z}$ & $\bar{z}$ & $\bar{z}$ & $\overline{-}$ & $\overline{-}$ & $\bar{z}$ & $\bar{z}$ & $\bar{z}+x+x$ & 12 & $\bar{z}$ & $\bar{z}+x+x$ & 19 \\
\hline $\begin{array}{l}\text { sossidulina subglobose } \\
\text { na, Guttulina }\end{array}$ & & $\stackrel{9}{1}$ & $3_{3}^{3}$ & $\begin{array}{l}8 \\
1\end{array}$ & $3^{3}$ & ${ }_{1}^{2}$ & 7 & & $\bar{z}$ r $\mathrm{x}$ - & $\bar{z}$ & & ${ }^{4}$ & $\overline{1}$ & $3_{1}^{3}$ & $\frac{1}{2}$ & $\begin{array}{l}1 \\
6\end{array}$ & $\overline{-}$ & - & $\overline{1}$ & 1 & & - & 2 & 2 & 6 & - & & $\bar{z}$ & $\bar{z}$ \\
\hline 500 & 20 & 14 & 13 & 16 & 12 & 6 & 4 & 8 & - & - & 4 & 7 & 8 & & ${ }_{4}^{2}$ & - & te & 2 & 4 & 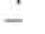 & 3 & & & & & & & & 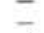 \\
\hline $\begin{array}{l}\text { Lina spp. } \\
\text { timodes }\end{array}$ & 1 & $\bar{z}$ & 6 & 6 & $a^{7}$ & 2 & $\frac{1}{2}$ & 2 & 2 & 1 & - & 6 & 9 & 11 & 10 & 32 & - & - & 3 & - & 2 & 9 & 28 & 9 & 18 & 4 & 3 & 2 & 5 \\
\hline $\begin{array}{l}\text { Melonis sp. } \\
\text { Notdosaria Stilostomella }\end{array}$ & ${ }_{9}^{3}$ & $\begin{array}{l}10 \\
25\end{array}$ & ${ }_{11}^{5}$ & $\stackrel{5}{19}$ & $\underset{18}{4}$ & $-_{4}$ & ${ }_{21}^{4}$ & $\overline{15}$ & ${ }_{11}^{2}$ & $\overline{12}$ & $-{ }_{6}$ & - & $\overline{2}$ & $\overline{4}$ & $\overline{10}$ & $\overline{2}$ & $\bar{z}+x+x-1$ & $\bar{z}+x \cdot+x+$ & 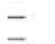 & $\bar{z}+\mathrm{r}-\mathrm{x}-\mathrm{z}$ & 13 & - & $\overline{12}$ & $\overline{14}$ & $\overline{26}$ & $\overline{12}$ & $\overline{-}$ & - & $z$ \\
\hline 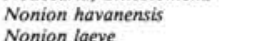 & $\overline{-}$ & $=$ & - & $\overline{-}$ & $=$ & $=$ & ${ }_{34}^{34}$ & 14 & 8 & - & 5 & $=$ & - & - & - & $=$ & $=$ & & $1-$ & $-\overline{-}$ & $\bar{z}$ & 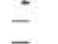 & - & $=$ & - & $\pi$ & - & - & to \\
\hline Vonion laeve & & $\bar{z}+x+$ & - & & - & $\bar{z}$ & & & $\bar{z}+x+$ & - & $=$ & $=$ & - & - & - & $=$ & $=$ & $=1$ & 11 & - & $\overline{-}$ & - & - & $\overline{-}$ & - & - & - & 5 & 8 \\
\hline uempyi & - & - & - & - & 1 & 3 & 3 & 9 & 2 & - & 4 & 2 & 2 & $=$ & $z$ & $=$ & - & $=$ & $=$ & - & z & 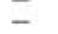 & $=$ & - & 1 & & $\bar{z}$ & & ${ }^{8}$ \\
\hline dorensis & 8 & $\frac{1}{12}$ & ${ }^{24}$ & 21 & ${ }^{4}$ & $\bar{z}$ & $\frac{7}{-}$ & 9 & 3 & 1 & 4 & $\bar{z}$ & $\frac{1}{-}$ & $\overline{-}$ & $\overline{-}$ & $=$ & $\overline{-}$ & $\overline{-}$ & - & $\overline{-}$ & $\bar{z}$ & - & $\bar{z}$ & $\bar{z}+$ & $\bar{z}$ & $\bar{z}$ & - & $=$ & - \\
\hline sosa & 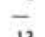 & & - & 1 & - & - & - & - & - & - & - & - & - & - & - & - & - & - & - & & - & - & - & - & 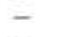 & 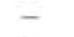 & - & - & E \\
\hline Dtalia curryi & 13 & ${ }_{-}^{5}$ & ${ }_{-}^{4}$ & $=$ & $-^{3}$ & -6 & ${ }_{-4}^{4}$ & $\underline{9}$ & 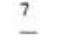 & -3 & 8 & 11 & 1 & $\overline{-}$ & $\frac{1}{-}$ & $\bar{z}$ & $\overline{-}$ & $\overline{-}$ & ${ }_{6}^{-}$ & $\overline{1}$ & $\overline{1}$ & - & $\overline{-}$ & $\bar{z}$ & - & $\bar{z}+$ & $=$ & $\bar{z}$ & $\overline{14}$ \\
\hline liming o & 4 & 1 & 2 & 3 & - & 1 & $\frac{3}{-}$ & $\frac{1}{-}$ & $=$ & 1 & - & $\overline{-}$ & $=$ & $=$ & $=$ & $=$ & - & - & $\bar{z}$ & & $\overline{8}$ & 3 & 23 & $\bar{z}$ & & 13 & & - & \\
\hline $\begin{array}{l}\text { rotelphidium sp. } \\
\text { retionata }\end{array}$ & $\overline{-}$ & $\overline{-}$ & $=$ & $\bar{z}$ & $\bar{z}$ & $\overline{-}$ & - & $=$ & $\bar{z}$ & $\overline{-}$ & $\bar{z}+$ & $\overline{-}$ & - & $\bar{z}$ & $\overline{-}$ & $\bar{z}$ & $\overline{-}$ & $\bar{z}+x$ & $\bar{z}$ & 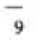 & 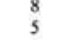 & 3 & 23 & $\overline{-}$ & 6 & 13 & ${ }^{4}$ & $-^{2}$ & 45 \\
\hline & & 8 & - & ${ }^{4}$ & - & - & 1 & - & - & - & 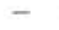 & - & - & 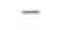 & - & - & - & - & 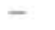 & & & & - & 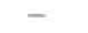 & & - & - & - & - \\
\hline queloba & 1 & 3 & ${ }_{3}^{6}$ & ${ }_{2}^{6}$ & $\underset{s}{11}$ & $3_{3}^{3}$ & $\overline{2}$ & $\bar{z}+x+$ & $\overline{2}$ & $\overline{2}$ & 6 & $-{ }_{2}$ & $\overline{2}$ & $\bar{z}+x+5$ & $\overline{1}$ & $\bar{z}$ & $\overline{-}$ & $\overline{-}$ & $\bar{z}$ & $\beth$ & $\overline{-}$ & $\bar{z}$ & $\Xi$ & $\overline{-}$ & 1 & $\overline{-}$ & $\overline{-}$ & $\bar{z}$ & - \\
\hline 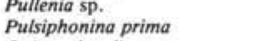 & ${ }^{2}$ & $\bar{z}$ & $\bar{z}$ & $-^{2}$ & $2^{2}$ & ${ }_{-}^{16}$ & $\bar{z}$ & $\overline{-}$ & $\bar{z}$ & $\bar{z}$ & $\bar{z}$ & $\overline{-}$ & $\frac{-5}{3}$ & $\frac{3}{2}$ & $\bar{z}+$ & $=$ & $\overline{-}$ & $\bar{z}+$ & - & - & $\overline{9}$ & 4 & 6 & 40 & - & $\begin{array}{l}1 \\
3 \\
\end{array}$ & $\bar{z}$ & - & - \\
\hline kana & $\overline{-}$ & $\overline{-}$ & - & - & $=$ & $\bar{z}$ & - & - & 8 & 3 & $\overline{17}$ & - & & & - & $5^{5}$ & - & - & & & & & & & & & & - & 2 \\
\hline ckana & 1 & 4 & 2 & 4 & 4 & 3 & & 2 & $\therefore$ & 3 & 1 & - & & - & $\bar{z}-\mathrm{s}+\mathrm{s}$ & $E$ & - & & & & & & & & & & & & E \\
\hline rifarina cuneata & - & - & - & - & - & - & - & - & - & - & - & - & - & - & 1 & 1 & - & - & - & - & 2 & - & & ${ }^{4}$ & 5 & 1 & & & - \\
\hline dentified & $\overline{14}$ & $\overline{11}$ & $\overline{10}$ & $\overline{13}$ & 9 & -9 & $\overline{7}$ & 8 & 2 & $\overline{8}$ & 5 & - & 8 & 8 & 5 & $\overline{13}$ & $\bar{z}$ & - & - & & & - & & & 5 & $\bar{s}$ & $E$ & 20 & \\
\hline tal & 137 & 139 & 114 & 126 & 113 & 106 & 143 & 132 & 95 & 52 & 108 & 112 & 105 & 112 & 108 & 101 & 15 & 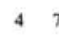 & 76 & 33 & 111 & 32 & 140 & 110 & 113 & 159 & 30 & 171 & 9 \\
\hline $\begin{array}{l}\text { umber of species } \\
\text { Ratiti planktonic to } \\
\text { benthic (x:1) }\end{array}$ & $\begin{array}{l}40 \\
99\end{array}$ & $\begin{array}{l}28 \\
99\end{array}$ & $\begin{array}{l}38 \\
99\end{array}$ & $\begin{array}{l}33 \\
99\end{array}$ & $\begin{array}{l}30 \\
99\end{array}$ & $\begin{array}{l}29 \\
99\end{array}$ & ${ }_{1.8}^{21}$ & $\begin{array}{l}19 \\
1.2\end{array}$ & $\begin{array}{l}17 \\
0.3\end{array}$ & $\begin{array}{r}14 \\
0\end{array}$ & ${ }_{0}^{23}$ & $\begin{array}{r}24 \\
3\end{array}$ & $\begin{array}{l}30 \\
2.3\end{array}$ & ${ }_{1.5}^{23}$ & $\begin{array}{l}30 \\
0.3\end{array}$ & $\begin{array}{l}25 \\
0\end{array}$ & $\begin{array}{l}6 \\
0\end{array}$ & 2 & 0.01 & $\begin{array}{l}6 \\
0\end{array}$ & ${ }_{0.05}^{26}$ & $\begin{array}{l}8 \\
0\end{array}$ & $\begin{array}{l}12 \\
0.02\end{array}$ & $\begin{array}{l}15 \\
0.031\end{array}$ & $\begin{array}{c}27 \\
0\end{array}$ & $\begin{array}{l}25 \\
0.3\end{array}$ & $\begin{array}{l}17 \\
0.07\end{array}$ & $\begin{array}{r}14 \\
0\end{array}$ & $\begin{array}{c}20 \\
0\end{array}$ \\
\hline
\end{tabular}


Table 11. Benthic foraminiferal census data, Hole 554A, Paleogene.

\begin{tabular}{|c|c|c|c|c|c|c|c|c|c|c|c|c|}
\hline \multirow[b]{2}{*}{ Species } & \multicolumn{7}{|c|}{ Oligocene } & \multicolumn{2}{|c|}{$\begin{array}{c}\text { late } \\
\text { Eocene }\end{array}$} & \multicolumn{3}{|c|}{$\begin{array}{c}\text { early } \\
\text { Eocene }\end{array}$} \\
\hline & $\begin{array}{l}\text { q } \\
\text { i }\end{array}$ & $\begin{array}{l}\bar{\Xi} \\
\tilde{+}\end{array}$ & $\begin{array}{l}\stackrel{8}{n} \\
\dot{r}\end{array}$ & $\begin{array}{l}\stackrel{\text { }}{+} \\
\dot{+}\end{array}$ & $\underset{\dot{J}}{0}$ & $\begin{array}{l}\& \\
\dot{n}\end{array}$ & $\begin{array}{l}\infty \\
\stackrel{0}{+} \\
\stackrel{\sim}{n}\end{array}$ & $\begin{array}{l}\vec{\Xi} \\
\dot{n}\end{array}$ & $\begin{array}{l}\simeq \\
\stackrel{+}{ \pm}\end{array}$ & $\underset{\sim}{U}$ & $\begin{array}{l}\stackrel{\infty}{\leftrightarrows} \\
\frac{5}{b}\end{array}$ & $\frac{\vec{b}}{b}$ \\
\hline Alabamina sp. & - & - & - & - & 1 & 2 & 2 & 8 & 2 & - & - & 3 \\
\hline Anomalinoides howelli & - & - & - & - & 4 & - & - & - & - & $\mathrm{a}$ & 38 & 9 \\
\hline Anomalinoides nobilis & - & - & - & - & - & - & - & - & - & a & - & - \\
\hline Anomalinoides sp. & - & - & - & - & 1 & - & - & - & - & - & 1 & 1 \\
\hline Astrononion guadelupae & 15 & - & - & 2 & - & - & - & - & - & - & - & - \\
\hline Bolivinopsis adamsi & - & - & - & - & - & - & - & - & - & - & 1 & - \\
\hline Brizalina subaenariensis & 2 & 2 & 3 & - & - & - & - & - & - & - & - & - \\
\hline Brizalina cf. B. thalmanni & 1 & - & - & - & - & - & - & - & - & - & - & - \\
\hline Brizalina sp. & - & 3 & 1 & 3 & - & - & 4 & - & 2 & - & 1 & - \\
\hline Bulimina alazanensis & - & - & - & 1 & 2 & - & - & - & - & - & - & - \\
\hline Bulimina trigonalis & - & - & - & - & - & - & - & - & 1 & - & - & - \\
\hline Bulimina tuxpamensis & - & - & - & 4 & - & - & - & 2 & - & a & 1 & - \\
\hline Bulimina sp. & 6 & 1 & 2 & 3 & - & 2 & - & - & - & - & 1 & - \\
\hline Ceratolamarckina sp. & - & - & - & - & - & - & - & - & - & - & 1 & - \\
\hline Cibicides, Cibicidina & - & - & - & - & - & 8 & - & - & 1 & $\mathrm{a}$ & 2 & 1 \\
\hline Cibicidoides alleni & - & - & - & - & - & - & - & - & - & - & 4 & - \\
\hline Cibicidoides bradyi & 2 & - & 1 & 6 & - & - & - & - & - & - & - & - \\
\hline Cibicidoides kullenbergi & 2 & 10 & 5 & - & - & - & - & - & - & - & - & - \\
\hline Cibicidoides spp. & - & - & 7 & 1 & 9 & 8 & 7 & 1 & 1 & - & - & - \\
\hline Eggerella bradyi & - & - & 1 & - & 1 & - & - & - & - & - & - & - \\
\hline Epistominella exigua & 9 & - & - & - & - & - & - & - & - & - & - & - \\
\hline Eponides umbonatus & - & - & - & - & 2 & - & - & - & - & - & 1 & - \\
\hline Fissurina, Lagena, Oolina & 4 & 2 & 2 & 3 & 3 & 1 & 7 & 1 & 1 & a & 1 & 1 \\
\hline Gavelinella semicribrata & - & - & - & - & - & - & - & - & - & - & 1 & - \\
\hline Globocassidulina subglobosa & 7 & 1 & 3 & 4 & 1 & 9 & 3 & - & 27 & - & 8 & - \\
\hline Globulina, Guttulina & - & 1 & - & 2 & 2 & - & - & - & 1 & $\mathrm{a}$ & 1 & - \\
\hline Gyroidinoides spp. & 21 & 9 & 31 & 13 & 10 & 16 & 13 & 10 & 8 & a & 11 & 6 \\
\hline Karreriela bradyi & - & - & - & - & 1 & - & - & - & - & - & - & - \\
\hline Melonis sp. & 11 & - & 20 & - & 4 & - & - & - & 1 & - & - & - \\
\hline Nodosaria spp./Stilostomella spp. & 22 & 45 & 18 & 25 & 27 & 16 & 17 & 33 & 20 & a & - & 1 \\
\hline Nonion havanensis & - & 18 & - & - & - & 17 & 18 & - & - & - & - & - \\
\hline Nonion sp. & - & - & - & - & - & 1 & - & 3 & - & - & 5 & - \\
\hline Nonionella sp. & - & - & 2 & 3 & 1 & - & - & - & - & - & - & - \\
\hline Oridorsalis ecuadorensis & 8 & - & - & 3 & 9 & 19 & 20 & 22 & 8 & - & - & - \\
\hline Oridorsalis umbonatus & - & 6 & 12 & - & - & 4 & - & - & - & - & - & - \\
\hline Osangularia rugosa & 1 & - & - & - & - & - & - & - & - & - & - & - \\
\hline Osangularia sp. & - & - & - & 2 & - & 7 & - & - & 1 & a & - & - \\
\hline Pararotalia calvezae & - & - & - & - & - & - & - & - & - & - & - & 1 \\
\hline Pleurostomella sp. & 4 & 4 & 4 & - & 6 & 1 & 5 & 2 & 5 & - & - & - \\
\hline Pullenia bulloides & 2 & - & 5 & 1 & 10 & - & - & 1 & - & - & - & - \\
\hline Pullenia osloensis & 3 & 5 & 11 & 6 & 6 & 11 & - & 3 & 3 & - & - & - \\
\hline Pullenia quinqueloba & 2 & 5 & 6 & 3 & 4 & 6 & - & - & - & - & 1 & - \\
\hline Pulsiphonina prima & - & - & - & - & - & - & - & - & - & a & 3 & - \\
\hline Spiroplectammina spectabilis & 14 & 10 & 10 & 8 & 2 & 2 & - & - & - & - & - & - \\
\hline Trifarina cuneata & - & - & - & - & - & - & - & - & 1 & - & - & - \\
\hline Uvigerina compressa & 1 & 3 & - & - & - & - & - & - & 1 & - & - & - \\
\hline Uvigerina sp. & - & 1 & 3 & 4 & 3 & - & 3 & 10 & 8 & - & - & - \\
\hline Unidentified & 3 & 14 & 1 & - & 7 & 8 & 5 & 10 & 17 & - & 18 & 12 \\
\hline Total & 138 & 142 & 157 & 113 & 116 & 139 & 109 & 116 & 109 & - & 100 & 35 \\
\hline Number of species & 33 & 26 & 27 & 32 & 36 & 33 & 18 & 22 & 29 & - & 34 & 18 \\
\hline $\begin{array}{l}\text { Ratio planktonic to } \\
\text { benthic }(x: 1)\end{array}$ & 16 & 19 & 12 & 99 & 99 & 16 & 10 & 46 & 7 & - & 1 & 0 \\
\hline
\end{tabular}

Note: Dash $=$ absent in assemblage count.

$\mathrm{a}=$ present.

the Pacific Ocean show that most of the benthic foraminifers are unaffected by the $3^{\circ} \mathrm{C}$ cooling inferred to have taken place at the Eocene/Oligocene boundary. Comparison with other sites, including some from the Atlantic Ocean, shows a similar lack of response from the fauna. From this Corliss concludes that the bottomwater circulation was established in the middle and late Eocene before the development of the cold bottom water.

There are two factors that make it difficult to assess the importance of this event in the study area: One is the poor late Eocene record and the frequency of hiatuses in the Eocene-Oligocene record, and the other is that the Eocene sediments are generally shelf or epibathyal. Vergnaud Grazzini, et al. (1979) interpreted the late Eocene bottom-water temperature at Site 403 to be $5^{\circ} \mathrm{C}$ (depth $\sim 600 \mathrm{~m}$, Murray, 1979). Evidence that the Paleogene North Atlantic was stratified has come from a study of the Bay of Biscay (Schnitker, 1979a). From the number of hiatuses and the discontinuous nature of the sedimentary record in the study area, it is evident that there were prolonged periods of very vigorous current activity which, in turn, may be indicative of the input of dense cold water which was corrosive and caused dissolution. 
Table 12. Benthic foraminiferal census data, Site 555, Paleogene.

\begin{tabular}{|c|c|c|c|c|c|c|c|c|c|c|c|c|c|c|c|c|c|c|c|c|c|c|c|c|c|c|c|c|c|}
\hline \multirow[b]{2}{*}{ Species } & \multicolumn{15}{|c|}{ early Eocene } & \multicolumn{14}{|c|}{ ?late Paleocene } \\
\hline & 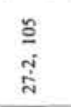 & $\frac{\tilde{m}}{\dot{\alpha}}$ & $\begin{array}{l}\text { తి } \\
\stackrel{\dot{m}}{-}\end{array}$ & 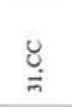 & 芯 & 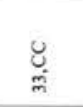 & 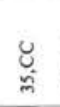 & लें & $\begin{array}{c}\mathscr{U} \\
\dot{\infty} \\
\text { d. }\end{array}$ & $\begin{array}{l}\text { U. } \\
\text { s+ }\end{array}$ & $\begin{array}{l}\mathscr{U} \\
\overrightarrow{7}\end{array}$ & 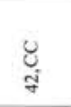 & $\begin{array}{l}\ddot{U} \\
\ddot{q}\end{array}$ & $\begin{array}{l}u \\
\dot{u} \\
j\end{array}$ & $\begin{array}{l}\ddot{U} \\
\dot{q}\end{array}$ & 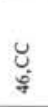 & 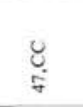 & $\begin{array}{l}\stackrel{u}{u} \\
\stackrel{q}{q}\end{array}$ & 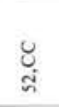 & $\underset{\sim}{\breve{~}}$ & $\begin{array}{l}\text { U } \\
\text { in }\end{array}$ & 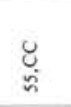 & 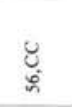 & $\underset{\dot{b}}{\tilde{b}}$ & $\begin{array}{l}\underset{d}{\infty} \\
\text { ஸ் }\end{array}$ & 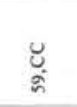 & $\stackrel{\breve{U}}{8}$ & $\underset{\square}{\stackrel{U}{0}}$ & $\begin{array}{l}E \\
\overrightarrow{6} \\
+ \\
6\end{array}$ \\
\hline Alabamina obtusa & - & 2 & - & 4 & - & - & - & - & - & - & 3 & - & 9 & 2 & - & 11 & 20 & 8 & - & - & - & - & 1 & 3 & 10 & 6 & 12 & - & 2 \\
\hline $\begin{array}{l}\text { Alabamina wilcoxensis } \\
\text { Anomalinoides acutus }\end{array}$ & 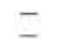 & $\overline{-}$ & $\overline{1}$ & \pm & 4 & $\overline{10}$ & $\overline{1}$ & $\overline{-}$ & $=$ & $=$ & $\overline{1}_{1}$ & $=$ & $2 \overline{27}$ & $\overrightarrow{17}$ & $\overline{11}$ & $\bar{z}+x+1$ & $\bar{z}+3$ & $=$ & $=$ & $=$ & $\bar{z}+$ & & $=$ & -7 & -7 & $\overline{2}$ & $\overline{6}$ & $\overline{9}_{9}$ & $\bar{u}$ \\
\hline $\begin{array}{l}\text { Anomalininodes acutus } \\
\text { Anomalinoides howelli }\end{array}$ & $=$ & $\overline{-}$ & 39 & 54 & $\overline{19}$ & ${ }^{10}$ & 2 & $=$ & $=$ & $\overline{2}$ & 2 & $=$ & 27 & -17 & 4 & $\overline{-}$ & $=$ & -5 & $=$ & $\overline{-}$ & $=$ & 7 & $=$ & $\frac{1}{7}$ & - & 2 & i & $\begin{array}{l}9 \\
6\end{array}$ & ${ }_{-}^{11}$ \\
\hline Anomalinoides nobilis & - & $-\overline{1}$ & 1 & 5 & 13 & 6 & - & - & - & - & 33 & 16 & - & 4 & 18 & - & 11 & 6 & - & - & 1 & 9 & 1 & ii & 22 & 2 & - & 8 & - \\
\hline $\begin{array}{l}\text { Anomalinoides sp. } \\
\text { Bolivinossis adams }\end{array}$ & - & 18 & 1 & $\bar{z}$ & - & - & - & $\overline{-}$ & $\bar{z}$ - & $\bar{z}$ - & - & - & 1 & - & $\overline{2}$ & - & 6 & - & $=$ & - & - & - & - & - & 4 & - & - & - & - \\
\hline $\begin{array}{l}\text { Bolivinopsis adamsi } \\
\text { Brizalina sp. }\end{array}$ & $\bar{z}+1+x$ & $\bar{z}$ & 1 & $\bar{s}$ & $\overline{3}$ & 1 & - & - & - & - & 1 & $\bar{z}+$ & 1 & - & 2 & - & $\overline{8}$ & - & - & - & - & - & - & 2 & - & - & 1 & $\overline{2}$ & - \\
\hline $\begin{array}{l}\text { Brizanina s. p. } \\
\text { Bulimina prainflata }\end{array}$ & $\bar{z}+1+x$ & $\overline{-}$ & $\overline{-}$ & $\frac{5}{-}$ & $\begin{array}{l}3 \\
1\end{array}$ & $\bar{z}$ & $\bar{z}$ & $\overline{-}$ & $\bar{z}+$ & $\bar{z}+1+$ & $=$ & $\bar{z}+x+$ & $=$ & $=$ & $\bar{z}+x+x$ & $\bar{\Xi}+x+1$ & -8 & $=$ & $=$ & $=$ & $=$ & $=$ & $\bar{z}+x+1$ & - & $=$ & $\Xi$ & $\bar{z}+x+3$ & ${ }_{-}^{2}$ & $=$ \\
\hline Bulimina trigonalis & - & 3 & - & 1 & 5 & 47 & - & - & - & - & - & - & - & - & - & - & - & - & - & - & - & - & - & 1 & - & - & - & - & - \\
\hline Bulimina tuxpamensis & - & - & - & 3 & 3 & - & - & - & - & - & - & - & - & - & - & $\overline{0}$ & $\bar{s}$ & - & - & - & - & - & - & - & - & $\overline{-}$ & - & $\overline{-}$ & - \\
\hline Cancris subconicus & - & - & - & - & - & - & - & - & 20 & - & - & - & - & - & - & 20 & 5 & 5 & - & 8 & - & - & 4 & 2 & - & 23 & 1 & 13 & 2 \\
\hline $\begin{array}{l}\text { Ceratolamarckina sp. } \\
\text { Cibicides of. C. westit }\end{array}$ & $\bar{z}+x+1$ & $\bar{z}+$ & 1 & $\overline{1}$ & $\bar{z}+x+x+$ & $\bar{z}+$ & $=$ & $\bar{z}+x+x$ & $\bar{z}+x+1+$ & $\bar{z}+9$ & ${ }^{8}$ & $=$ & $\overline{-}$ & $=$ & $\bar{z}+x+x$ & $\bar{z}+$ & $\overline{-}$ & $=$ & $=$ & $=$ & $=$ & $\overline{3}$ & $\underline{5}$ & $\begin{array}{l}4 \\
1\end{array}$ & 7 & $=$ & $\overline{18}$ & $=$ & $\overline{77}$ \\
\hline Cibicides/Cibicidina & $\overline{-}$ & - & $\overline{1}$ & 8 & $\overline{6}$ & - & $\overline{1}$ & $\overline{-}$ & $\bar{z}+x+1$ & $=$ & $=$ & $=$ & $\overline{3}$ & $\bar{z}+$ & $\bar{z}+x+x+$ & $\overline{-}$ & $=$ & 2 & $\overline{-}$ & - & $=$ & - & $\overline{1}$ & - & $i$ & 1 & 18 & 2 & ${ }_{-}^{77}$ \\
\hline Cibicidoides alleni & - & - & - & - & - & 3 & - & - & - & - & 8 & 10 & - & 13 & 5 & - & 1 & - & - & - & - & - & - & - & - & - & - & - & - \\
\hline Cibicidoides succedens & - & 5 & - & - & 2 & - & - & - & - & - & - & - & - & - & - & - & - & - & - & - & - & - & - & - & - & - & - & - & - \\
\hline Cibicidoides spp. & - & 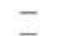 & - & 3 & 14 & 2 & - & - & - & - & $\overline{21}$ & $-\overline{-}$ & $\overline{23}$ & 1 & - & - & 1 & -3 & $\overline{-}$ & -7 & 7 & - & $\overline{2}$ & - & - & $\bar{z}$ & - & - & - \\
\hline $\begin{array}{l}\text { Cribrostomo } \\
\text { Elphidium ho hen }\end{array}$ & $\bar{z}+x+1$ & $=$ & $=$ & & $\overline{-}$ & 1 & 2 & $\bar{z}+$ & $\bar{z}$ & 1 & 21 & 41 & 23 & 3 & 1 & - & 3 & ${ }_{-3}^{3}$ & $\bar{z}+$ & 18 & 1 & 4 & 2 & 3 & 2 & - & 3 & 1 & 9 \\
\hline $\begin{array}{l}\text { Elphiditur } \\
\text { Epistomi }\end{array}$ & $=$ & $\overline{-}$ & $\overline{-}$ & $\bar{z}+x+x+1$ & $\overline{-}$ & $\overline{1}$ & $=$ & $=$ & $z$ & $=$ & $\overline{3}$ & $=$ & $\bar{z}+$ & $=$ & $\overline{1}$ & $=$ & 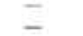 & $\overline{-}$ & -1 & $=$ & $=$ & $\overline{-}$ & $\overline{-}$ & $=$ & $\overline{-}_{3}$ & $\overline{1}$ & ${ }^{4}$ & $=$ & $\overline{-}$ \\
\hline Eponides umbonatus & - & 1 & - & - & 2 & - & $=$ & - & - & 6 & - & 9 & - & - & - & - & - & - & - & - & - & - & - & - & 9 & - & $\overline{2}$ & $=$ & $\bar{z}$ \\
\hline Eponides spp. & - & - & - & 2 & 5 & - & - & - & - & - & 7 & 3 & 2 & 3 & 34 & - & 1 & - & - & 2 & 22 & 68 & 2 & 2 & 1 & - & 2 & 9 & 7 \\
\hline ena, Oolina & 2 & 1 & 14 & 5 & 2 & - & - & - & - & - & - & - & - & - & - & - & 2 & - & - & - & - & - & 2 & $i$ & - & 1 & 2 & 1 & - \\
\hline 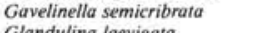 & - & - & 1 & 5 & 5 & 2 & - & - & - & - & - & - & - & - & - & - & - & - & - & - & - & - & - & - & - & - & - & - & - \\
\hline $\begin{array}{l}\text { Glandulina } \\
\text { Githocassid }\end{array}$ & - & $\bar{z}+x-1+x$ & $\overline{1}$ & $\overline{3}$ & $\overline{6}$ & - & $=$ & $\bar{z}$ & $=$ & 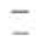 & $=$ & - & - & 7 & - & - & $\bar{z}$ & - & $=$ & $=$ & - & z & - & - & - & $\overline{-}$ & $\overline{-}$ & $=$ & - \\
\hline $\begin{array}{l}\text { Globoca } \\
\text { Globulin }\end{array}$ & $=$ & $\overline{1}$ & 5 & 1 & -6 & $\overline{-}$ & $\overline{-}$ & $=$ & $=$ & $=$ & I & $\overline{-}$ & $\overline{1}$ & -2 & $=$ & $=$ & $\overline{-}$ & -2 & $\overline{-}$ & $=$ & $=$ & $\overline{3}$ & $\overline{-}$ & - & $\tau_{2}$ & $=$ & $\overline{-}$ & $=$ & $\overline{1}$ \\
\hline $\begin{array}{l}\text { Globulina, G } \\
\text { Gyroidinoide }\end{array}$ & 6 & 9 & 15 & 2 & $\overline{1}$ & $\overline{2}$ & - & - & - & - & - & 4 & - & 23 & 33 & - & 9 & - & - & - & - & 6 & 2 & 4 & - & - & $\overline{2}$ & $=$ & $\frac{1}{-}$ \\
\hline Lenticulina spp. & 1 & - & 3 & 3 & 5 & 2 & 2 & - & - & 3 & 11 & 1 & 1 & 13 & 2 & 2 & 31 & 65 & - & 1 & 5 & 11 & 4 & 1 & 7 & - & 4 & - & 2 \\
\hline ides applinae & - & - & - & - & i & $i$ & $i$ & - & - & - & 3 & 3 & - & - & - & - & - & 3 & - & - & 1 & 4 & 1 & 4 & I & 4 & - & 12 & - \\
\hline milioi & - & - & - & $\overline{-}$ & - & - & - & - & 1 & - & - & - & - & - & - & 1 & - & 2 & - & 1 & 11 & 1 & 21 & 4 & 3 & - & - & - & - \\
\hline Nodo & 4 & 5 & 3 & 10 & 3 & 6 & 1 & - & - & 1 & 8 & 3 & 30 & 13 & 1 & - & 3 & 4 & - & 1 & 1 & - & 1 & - & - & 1 & 2 & - & 1 \\
\hline $\begin{array}{l}\text { Nonior } \\
\text { Nanion }\end{array}$ & 9 & - & 4 & - & - & - & - & $\bar{z}+1$ & - & $\bar{z}$ & - & $\bar{z}$ & - & - & - & - & - & -7 & - & - & - & - & - & - & - & - & $\overline{0}$ & - & - \\
\hline $\begin{array}{l}\text { Nonion lat } \\
\text { Nonionell }\end{array}$ & $=$ & $\bar{z}$ & 1 & 1 & $\overline{-}$ & $=$ & $=$ & $=$ & $\overline{67}$ & - & - & $\overline{-}$ & $\overline{-}$ & $=$ & $\overline{-}$ & $\bar{z}$ & $\overline{-}$ & 7 & $\overline{-}$ & $\bar{z}$ & - & $\overline{-}$ & - & $\bar{z}$ & - & $\bar{z}$ & 9 & $\bar{z}$ & - \\
\hline $\begin{array}{l}\text { Nonionerlal } \\
\text { Nonionella }\end{array}$ & 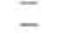 & $\bar{z}$ & $=$ & $\bar{z}$ & $\overline{-}$ & $\bar{z}$ & $=$ & $\bar{z}$ & $\begin{array}{l}67 \\
15\end{array}$ & $z$ & $\overline{-}$ & $\overline{2}$ & $\overline{2}$ & $\tau_{2}$ & $\bar{z}$ & $z$ & $\overline{5}$ & $=$ & $=$ & $=$ & $\bar{z}$ & $\bar{z}$ & $\overline{-}$ & $\overline{12}$ & $\overline{14}$ & $\overline{19}$ & $\overline{1}$ & -9 & $\overline{-}$ \\
\hline Osar & 58 & 32 & - & - & - & - & - & - & - & - & - & - & - & - & - & - & - & - & - & - & - & - & - & - & - & - & - & - & - \\
\hline & $\bar{z}$ & - & - & & - & & - & - & - & - & - & $\overline{10}$ & $\overline{10}$ & - & $\overline{9}$ & - & $\overline{1}$ & - & - & - & $\overline{1}$ & - & $\overline{4}$ & $\overline{32}$ & - & $\overline{69}$ & $\overline{7}$ & & - \\
\hline ovata & - & - & T & 2 & - & 15 & $=$ & $\overline{3}$ & 5 & $\bar{z}$ & 1 & 12 & 12 & 5 & 9 & - & 1 & 2 & $\overline{14}$ & $=$ & 1 & - & 54 & 32 & 2 & 69 & 17 & 51 & 1 \\
\hline 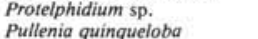 & $\overline{3}$ & $\overline{-}$ & - & $\overline{2}$ & $\overline{1}$ & $\overline{-}$ & $=$ & -3 & $=$ & $=$ & $\bar{z}$ & $=$ & $\overline{-}$ & $=$ & $\overline{-}$ & 18 & $\overline{-}$ & $=$ & 141 & $=$ & $=$ & $=$ & $\bar{z}$ & $\bar{z}$ & $\overline{-}$ & $\bar{z}$ & $\overline{-}$ & $=$ & $=$ \\
\hline Puls & - & - & - & $i$ & 5 & 6 & - & - & - & - & 2 & 4 & 2 & 5 & 1 & - & - & - & - & - & - & - & - & 1 & - & - & 8 & 2 & - \\
\hline bilitis & 1 & - & - & - & - & - & - & - & - & - & - & - & - & - & & - & - & - & - & - & - & - & - & - & - & - & - & - & - \\
\hline & - & - & - & 2 & 4 & - & - & - & - & - & - & - & - & - & - & - & -7 & - & - & - & - & - & - & - & - & - & - & - & - \\
\hline & $\bar{z}$ & - & $=$ & $=$ & - & $=$ & - & $=$ & - & - & $=$ & - & - & - & $=$ & $=$ & 3 & - & $=$ & $\bar{z}$ & $=$ & $=$ & - & - & $\overline{10}$ & $\bar{z}$ & $\bar{z}$ & - & $\overline{-}$ \\
\hline $\begin{array}{l}\text { Uvigerina breviata } \\
\text { Unidentified }\end{array}$ & $\overline{35}$ & 25 & $\overline{7}$ & $\overline{16}$ & $\overline{5}$ & $\overline{-}$ & $\overline{-}$ & $\bar{z}$ & $\bar{z}$ & $\overline{1}$ & $\bar{T}_{1}$ & $\bar{z}$ & $\overline{1}$ & $=$ & $\bar{z}$ & $\bar{z}$ & $\overline{5}$ & $\tau_{1}$ & $\overline{-}_{4}$ & $\bar{z}$ & $\overline{1}$ & $\bar{z}$ & $\bar{z}$ & $\begin{array}{l}1 \\
2\end{array}$ & $\begin{array}{l}10 \\
10\end{array}$ & $\bar{z}$ & $\overline{3}$ & $2^{2}$ & $\bar{z}$ \\
\hline Total & 118 & 102 & 100 & 140 & 115 & 120 & 10 & 3 & 108 & 15 & 113 & 105 & 113 & 113 & 122 & 52 & 115 & 115 & 146 & 31 & 44 & 116 & 101 & 108 & 115 & 129 & 106 & 127 & 113 \\
\hline $\begin{array}{l}\text { Number of species } \\
\text { Ratio planktonic to } \\
\text { benthic }(x: 1)\end{array}$ & $\begin{array}{l}23 \\
0.2\end{array}$ & $\begin{array}{l}16 \\
0.07\end{array}$ & $\begin{array}{c}27 \\
0.8\end{array}$ & $\begin{array}{c}39 \\
2.6\end{array}$ & $\begin{array}{c}30 \\
0.3\end{array}$ & $\begin{array}{l}17 \\
0.02\end{array}$ & $\begin{array}{l}7 \\
0\end{array}$ & $\begin{array}{l}1 \\
0\end{array}$ & $\begin{array}{l}5 \\
0\end{array}$ & $\begin{array}{l}8 \\
0\end{array}$ & $\begin{array}{r}19 \\
0\end{array}$ & $\begin{array}{l}12 \\
0.01\end{array}$ & $\begin{array}{l}14 \\
0.03\end{array}$ & $\begin{array}{r}16 \\
0\end{array}$ & $\stackrel{16}{0.02}$ & $\begin{array}{l}6 \\
0\end{array}$ & $\stackrel{22}{0.03}$ & $\begin{array}{r}14 \\
0\end{array}$ & $\begin{array}{l}3 \\
0\end{array}$ & $\begin{array}{l}6 \\
0\end{array}$ & $\begin{array}{r}11 \\
0\end{array}$ & $\stackrel{13}{0.02}$ & $\stackrel{16}{16}_{0.01}$ & $\begin{array}{r}25 \\
0\end{array}$ & $\begin{array}{r}25 \\
0\end{array}$ & $\stackrel{12}{0.01}$ & ${ }_{0.02}^{24}$ & $\begin{array}{r}16 \\
0\end{array}$ & $\begin{array}{r}{ }^{11} \\
0.03\end{array}$ \\
\hline & $\mathrm{CC}$ & ${ }^{C} C_{\text {. }}$ & 7 & 74 & & & & & & & & & & & & & & & & & & & & & & & & & \\
\hline
\end{tabular}

Note: Dash $=$ Absent in assemblage count. 
Table 13. Paleogene species groups used in the varimax factor analysis.

\begin{tabular}{ll}
\hline 1. Alabamina obtusa & 17. Gyroidinoides spp. \\
2. Alabamina spp. & 18. Lagena, Fissurina, Globulina, Guttulina \\
3. Anomalinoides acutus & 19. Lenticulina spp. \\
A. spp., A. howelli & 20. Melonis spp. \\
Cibicides, Cibicidina & 21. Nodosaria/Stilostomella \\
4. Gavelinella semicribrata & 22. Nonion laeve \\
5. Anomalinoides nobilis & 23. Nonion havanensis \\
6. Bulimina tuxpamensis & 24. Nonionella/Florilus \\
7. Bulimina \& Brizalina & 25. Nuttallides truempyi \\
8. Cancris subconicus & 26. Oridorsalis spp. \\
9. Cibicidoides alleni & 27. Osangularia spp. \\
10. Cibicidoides spp. & 28. Pleurostomella spp. \\
11. Cribrostomoides/Trochammina & 29. Protelphidium spp. \\
12. Elphidium hiltermanni & 30. Pullenia spp. \\
13. Epistominella vitrea & 31. Pulsiphonina prima \\
14. Eponides spp. & 32. Spiroplectammina spectabilis \\
15. Gaudryina spp. & 33 . Uvigerina spp. \\
16. Globocassidulina subglobosa & 34. Praeglobobulimina ovata
\end{tabular}

Table 14. Paleogene assemblages represented by varimax factors (factor scores $\times 100$ ).

Varimax factor I

62.9 Nodosaria/Stilostomella spp.

39.5 Gyroidinoides spp.

37.5 Pullenia spp.

33.9 Oridorsalis spp.

24.0 Cibicidoides spp.

Varimax factor II

96.9 Anomalinoides acutus, $A$. howelli, Cibicides, Cibicidina

Varimax factor III

89.7 Praeglobobulimina ovata

22.4 Nonionella sp.

21.3 Cancris subconicus

Varimax factor IV

-64.2 Lenticulina spp.

- 44.8 Cribrostomoides/Trochammina

-33.4 Anomalinoides nobilis

-24.1 Nodosaria/Stilostomella

- 20.1 Cibicidoides alleni

- 19.2 Alabamina obtusa

Varimax factor $\mathrm{V}$

-54.8 Alabamina sp.

-51.8 Osangularia sp.

-43.8 Bulimina tuxpamensis

-29.6 Nonion havanensis

-25.5 Cibicidoides spp.

-18.1 Nuttallides truempyi

As much of the Norwegian Sea Paleogene record has been badly affected by dissolution, it is tempting to invoke the introduction of cool Norwegian Sea water into the area as the cause of both the vigorous bottom circulation and the dissolution. However, the influence of Antarctica cannot be ignored, for this played a major role in Paleogene circulation of the South Atlantic, and van Andel et al. (1977) describe the change in the deep water circulation there as from a preglacial to a protoglacial pattern.

Faunal similarities within comparable depth zones show that in shelf seas there was faunal communication between such widely separated areas as Rockall Plateau,
Vøring Plateau, and the North Sea during the early Eocene. In deeper water there was communication between Orphan Knoll and the southern margin of Rockall Plateau (Sites 405,406 ) in early and middle Eocene times, and between Rockall margin (Sites 552, 553, 554), Hatton-Rockall Basin (Sites 116, 117), and the IcelandFaeroes Ridge (Site 352) in Oligocene times.

Recent reviews of the Cenozoic development of deep circulation in the North Atlantic have favored an input of cold water from the Norwegian Sea by late Eocene to early Oligocene times (Berggren and Hollister, 1974; Berggren and Schnitker, in press; Miller and Tucholke, in press). The interpreted existence of NADW assemblages (based on the Q-mode varimax factor analyses from at least early Miocene time is consistent with, and lends some support to, this interpretation.

\section{ACKNOWLEDGMENTS}

I am grateful to Ms. J. Weston for making available her data on the modern benthic Foraminifera of the Northeast Atlantic, for carrying out much of the computing, and for discussion. Problems of taxonomy were discussed with Dr. R. G. Douglas and Ms. F. Woodruff of the University of Southern California; Drs. B. Corliss, P. Belanger, and K. Miller of Woods Hole Oceanograhic Institution; Dr. W. Poag of the U.S. Geological Survey; and Dr. D. Schnitker of the University of Maine. Dr. R. Riding, University College of Wales, Cardiff, and Dr. G. Elliott, British Museum (Natural History) identified the dasyclad alga. The author is grateful for financial support from the Natural Environment Research Council to make visits to research institutes in the United States. Ms. G. Wright typed the manuscript and Mr. J. Jones prepared the photographs in Plates 1 to 4 from negatives taken by the author. Dr. J. R. Haynes, University College of Wales, Aberystwyth, and Dr. D. Schnitker, University of Maine, kindly commented on the manuscript. The reviewers were Dr. W. Poag, U.S.G.S., and Dr. B. Corliss, Woods Hole.

\section{REFERENCES}

Alvinierie, J., Caralp, H., Latouche, C., Moyes, J., and Vigneaux, M., 1978. Apport à la connaissance de la paléohydrologie de l'Atlantique nord-oriental pendant le Quaternaire terminal. Oceanol. Acta, 1:87-98.

Belanger, P. E., and Streeter, S. S., 1980. Distribution and ecology of benthic foraminifera in the Norwegian-Greenland Sea. Mar. Micropaleontol., 5:401-428.

Benson, R. H., 1975. The origin of the psychrosphere as recorded in changes of deep-sea ostracod assemblages. Lethaia, 8:69-83

Berger, W. H. and Winterer, E. L., 1974. Plate stratigraphy and the fluctuating carbonate line. In Hsü, K. J., and Jenkyns, H. (Eds.), Pelagic Sediments on Land and Under the Sea. Spec. Publ. Int. Assoc. Sedimentol., 1:11-48.

Berggren, W. A., 1974. Late Paleocene-Early Eocene benthonic foraminiferal biostratigraphy and paleoecology of Rockall Bank. Micropaleontology, 20:426-448.

Berggren, W. A., and Aubert, J., 1976a. Eocene benthonic foraminiferal biostratigraphy and paleobathymetry of Orphan Knoll (Labrador Sea). Micropaleontology, 22:327-346. , 1976b. Late Paleogene (Late Eocene and Oligocene) benthonic foraminiferal biostratigraphy and paleobathymetry of Rockall Bank and Hatton-Rockall Basin. Micropaleontology, 22:307-326.

Berggren, W. A., and Hollister, C. D., 1974. Paleogeography, paleobiography and the history of circulation of the Atlantic Ocean. Soc. Econ. Paleontol. Mineral. Mem., 20:126-186.

Berggren, W. A., and Schnitker, D., in press. Cenozoic marine environments in the North Atlantic and Norwegian-Greenland Sea.

Boltovskoy, E., and Wright, R., 1976. Recent Foraminifera: The Hague (Junk), pp. 1-515.

Bott, M., Talwani, M., Thiede, J., and Saxov, S. (Ed.), in press. The structure and development of the Greenland-Scotland Ridge: New methods and concepts. Proc. NATO Advanced Research Institute.

Cooper, L. H. N., 1955. Deep water movements in the North Atlantic as a link between climatic changes around Iceland and biological 
productivity of the English Channel and Celtic Sea. J. Mar. Res., $4: 347-362$.

Corliss, B. H., 1981. Deep-sea benthonic foraminiferal fauna turnover near the Eocene/Oligocene boundary. Mar. Micropaleontol., 6: 367-384.

Culver, S. J., and Buzas, M. A., 1980. Distribution of Recent benthic foraminifera off the North American Atlantic coast. Smithsonian Contrib. Mar. sci., 6:1-512.

Douglas, R., and Woodruff, F., 1981. Deep sea benthic foraminifera. In: Emiliani, C. (Ed.), The Sea (Vol. 7): New York (Plenum), 1233-1327.

Haake, F. W., Coulbourn, W. T., and Berger, W. H., 1982. Benthic Foraminifera: depth distribution and redeposition. In von Rad, V., Hinz, K., Sarnthein, M., and Seibold, E. (Eds.), Geology of the Northwest African Continental Margin: Berlin (Springer Verlag), pp. 632-657.

Jenkins, D. G., and Murray, J. W., 1981. Stratigraphical Atlas of Fossil Foraminifera: Chichester (British Micropalaeontological Series, and Ellis Horwod), pp. 1-310.

Kennett, J. P., and Shackleton, N. J., 1976. Oxygen isotope evidence for the development of the psychrosphere $38 \mathrm{Myr}$ ago. Nature, 260:513-515.

Lagoe, M. B., 1977. Recent benthic foraminifera from the Central Arctic Ocean. J. Foram. Res., 7:106-129.

Laughton, A. S., Berggren, W. A., et al., 1972. Init. Repts. DSDP, 12: Washington (U.S. Govt. Printing Office).

Lohman, G. P., 1978. Abyssal benthonic Foraminifera as hydrographic indicators in the western South Atlantic Ocean. J. Foram. Res., 8:6-34.

Lonsdale, P., and Hollister, C. D., 1979. A near-bottom traverse of Rockall Trough hydrographic and geological inferences. Oceanol. Acta, 2:11-105.

Lutze, G. F. 1980. Depth distribution of benthic foraminifera on the continental margin off NW Africa. Meteor Forsch.-Ergebnisse, 32:31-80.

Miller, K. G., and Lohmann, G. P., 1982. Environmental distribution of Recent benthic foraminifera in the northeast United States continental slope. Geol. Soc. Am. Bull., 93:200-206.

Miller, K. G., and Tucholke, B. C., in press. Development of Cenozoic abyssal circulation south of the Greenland-Scotland Ridge. Proc. NATO Advanced Research Institute.

Moore, T. C., Jr., van Andel, Tj. H., Sancetta, C., and Pisias, N., 1978. Cenozoic hiatuses in pelagic sediments. Micropaleontology, 24:113-138.

Murray, J. W., 1973. Distribution and Ecology of Living Benthic Foraminiferids: London (Heinemann).

1976a. Comparative studies of living and dead benthic foraminiferal distributions. In Hedley, R. H., and Adams, C. G., (Eds.), Foraminifera, 2:45-109.

$1976 \mathrm{~b}$. A method of determining proximity of marginal seas to an ocean. Mar. Geol., 22:103-109.

1979. Cenozoic biostratigraphy and paleoecology of Sites 403 to 406 based on the foraminifers. In Montadert, L., Roberts, D. G., et al., Init. Repts. DSDP, 48: Washington (U.S. Govt. Printing Office), 415-430.

Murray, J. W., and Wright, C. A., 1974. Palaeogene Foraminiferida and palaeoecology, Hampshire and Paris Basins and the English Channel. Spec. Pap. Palaeontol., 14:1-129.

Phleger, F. B., 1952. Foraminiferal distribution in some sediment samples from the Canadian and Greenland Arctic. Contrib. Cushman Found. Foram. Res., 3:80-89.
Phleger, F.B., Parker, F. L., and Peirson, J. F., 1953. North Atlantic Foraminifera. Rept. Swed. Deep-Sea Exped., 7:1-122.

Schafer, C. T., and Cole, F. E., 1982. Living benthic foraminifera distributions on the continental slope and rise east of Newfoundland, Canada. Geol. Soc. Am. Bull., 93:207-217.

Schnitker, D., 1974. West Atlantic abyssal circulation during the past 120,000 years. Nature, 248:385-387. , 1979a. Cenozoic deep water benthic foraminifers, Bay of Biscay. In Montadert, L., Roberts, D. G., et al., Init. Repts. DSDP, 48: Washington (U.S. Govt. Printing Office), 377-413. 1979b. The deep waters of the western North Atlantic during the past 24,000 years, and the re-initiation of the Western Boundary Undercurrent. Mar. Micropaleontol., 4:265-280. 1980. Quaternary deep-sea benthic foraminifers and bottom water masses. Ann. Rev. Earth Planet. Sci., 8:343-370.

Schrader, H. J., Bjorklund, K., Manum, S., Martini, E., and van Hinte, J., 1979. Cenozoic biostratigraphy, physical stratigraphy, and paleoceanography in the Norwegian-Greenland Sea, DSDP Leg 38 paleontological synthesis. In Talwani, M., Udintsev, G., et al., Init. Repts. DSDP, 38: Washington (U.S. Govt. Printing Office), 1197-1211.

Sen Gupta, B. K., Lee, R. F., and May, M. S., 1981. Upwelling and an unusual assemblage of benthic foraminifera on the northern Florida continental slope. J. Paleontol., 55:853-857.

Streeter, S. S., 1973. Bottom water and benthonic Foraminifera in the North Atlantic-Glacial-Interglacial cycles. Quat. Res., 3:131-141.

Streeter, S. S., and Lavery, S. A., 1982. Holocene and latest glacial benthic foraminifera from the slope and rise off eastern North America. Geol. Soc. Am. Bull., 93:190-199.

Talwani, M., Udintsev, G., et al., 1976. Init. Repts. DSDP, 38: Washington (U.S. Govt. Printing Office).

Vail, P. R., Mitchum, R. M., and Thompson, S., 1977. Seismic stratigraphy and global changes of sea level, Pt. 4: global cycles of relative changes of sea level. Am. Assoc. Pet. Geol Mem., 26:83-97.

van Andel, Tj. H., Thiede, J., Sclater, J. G., and Hay, W. W., 1977. Depositional history of the South Atlantic Ocean during the last 125 million years. J. Geol., 85:651-698.

van Hinte, J. E., 1976. Sites 336 and 352, In Talwani, M., Udintsev, G., et al., Init. Repts. DSDP, 38: Washington (U.S. Govt. Printing Office), 23-116.

Vergnaud Grazzini, C., Müller, C., Pierre, C., Létolle, R., and Peypouquet, J. P., 1979. Stable isotopes and Tertiary paleontological paleoceanography in the Northeast Atlantic. In Montadert, L., Roberts, D. G., et al., Init. Repts. DSP, 48: Washington (U.S. Govt. Printing Office), 475-487.

Williams, C. B., 1964. Patterns in the Balance of Nature: London (Academic Press).

Worthington, L. V., 1970. The Norwegian Sea as a Mediterranean basin. Deep-Sea Res., 17:77-84. 1976. On the North Atlantic circulation. John Hopkins Oceanogr. Ser., 6:1-110.

Worthington, L. V., and Wright, W. R., 1970. North Atlantic Ocean Atlas of potential temperature, and salinity in the deep water, including temperature, salinity and oxygen profiles from the "Erika Dan" cruise of 1962. Woods Hole Oceanogr. Inst. Atlas Ser. (Vol. 2).

Date of Acceptance: May 24, 1983 
Table 15. Distribution of Q-mode varimax factors in the Paleogene samples.

\begin{tabular}{|c|c|c|c|c|c|c|c|c|c|c|c|c|c|c|c|c|c|c|c|c|}
\hline & \multicolumn{4}{|c|}{$\begin{array}{c}\text { late } \\
\text { Paleocene }\end{array}$} & \multicolumn{4}{|c|}{$\begin{array}{l}\text { early } \\
\text { Eocene }\end{array}$} & \multirow{2}{*}{\multicolumn{4}{|c|}{$\begin{array}{c}\begin{array}{c}\text { middle } \\
\text { Eocene }\end{array} \\
\text { Sites }\end{array}$}} & \multicolumn{4}{|c|}{$\begin{array}{c}\text { late } \\
\text { Eocene }\end{array}$} & \multicolumn{4}{|c|}{ Oligocene } \\
\hline & & & & & & & & & & & & & & & & & & & & \\
\hline & 552 & 553 & 554 & 555 & 552 & 553 & 554 & 555 & 552 & 553 & 554 & 555 & 552 & 553 & 554 & 555 & 552 & 553 & 554 & 555 \\
\hline Marginal & & & & & & $\begin{array}{l}\text { II } \\
\text { IV }\end{array}$ & & & & & & & & & & & & & & \\
\hline Inner shelf & & $\begin{array}{l}\text { II } \\
\text { III } \\
\text { IV }\end{array}$ & & $\begin{array}{l}\text { II } \\
\text { III } \\
\text { IV }\end{array}$ & & II & & $\begin{array}{l}\text { II } \\
\text { III } \\
\text { IV }\end{array}$ & & & & & & & & & & & & \\
\hline Mid shelf & & & & & II & $\begin{array}{c}\text { I } \\
\text { II } \\
\text { IV } \\
\text { V }\end{array}$ & II & $\begin{array}{l}\text { II } \\
\text { V }\end{array}$ & & & & & & & & & & & & \\
\hline Outer shelf & & & & & & $\begin{array}{l}\text { II } \\
\text { V }\end{array}$ & & $\begin{array}{l}\text { II } \\
\text { V }\end{array}$ & & & & & & & & & & & & \\
\hline Slope $>700 \mathrm{~m}$ & & & & & & $\begin{array}{l}\text { I } \\
\text { V }\end{array}$ & & & $\begin{array}{l}\text { I } \\
\text { V }\end{array}$ & $\begin{array}{l}\text { I } \\
\text { V }\end{array}$ & & & & & I & & & & I & \\
\hline Slope $>1500 \mathrm{~m}$ & & & & & & & & & & & & & & & & & I & $\begin{array}{l}\text { I } \\
\text { V }\end{array}$ & & \\
\hline
\end{tabular}

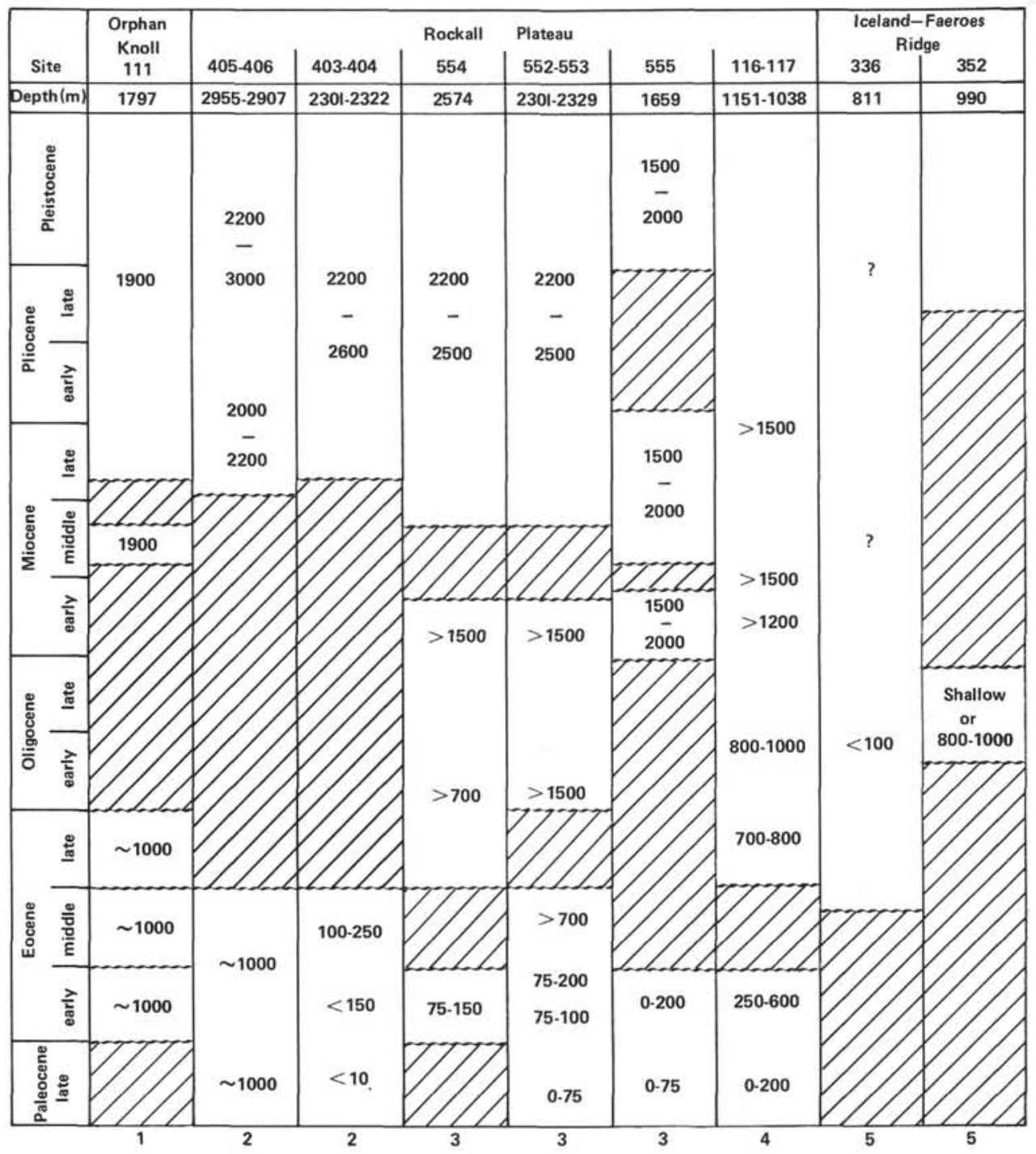

Figure 11. Depths of deposition (in meters) of North Atlantic DSDP sites based on analysis of benthic foraminiferal assemblages. (1. Berggren, Laughton et al., 1972; Berggren and Aubert, 1976a; 2. Murray, 1979; 3. this paper; 4. Berggren, Laughton, et al., 1972; Berggren, 1974; Berggren and Aubert, 1976b; 5. van Hinte, 1976.) 
Table 16. Comparison of Quaternary and Neogene assemblages throughout the North Atlantic.

\begin{tabular}{|c|c|c|c|c|}
\hline & & $\begin{array}{c}\text { Rockall margins } \\
2300-3000 \mathrm{~m} \\
\text { Sites } 403-406,552-554\end{array}$ & $\begin{array}{c}\text { Rockall Plateau; } \\
\text { Orphan Knoll } \\
1000-1800 \mathrm{~m} \\
\text { Sites } 116,117,555,111\end{array}$ & $\begin{array}{l}\text { Iceland-Faeroes- } \\
\text { Ridge } \\
800-1000 \mathrm{~m} \\
\text { Sites } 336,352\end{array}$ \\
\hline $\begin{array}{l}\text { Pleisto- } \\
\text { cene }\end{array}$ & Glacial? & $\begin{array}{l}\text { Epistominella exigua } \\
\text { Gyroidinoides spp. } \\
\text { Oridorsalis umbonatus } \\
\text { Planulina wuellerstorfi } \\
\text { Pullenia spp. } \\
\text { Pyrgo murrhina } \\
\text { Melonis pompilioides } \\
\text { Cassidulina teretis } \\
\text { Triloculina frigida }\end{array}$ & $\begin{array}{l}\text { Sigmoilopsis } \\
\quad \text { schlumbergeri } \\
\text { Pyrgo murrhina } \\
\text { Uvigerina } \text { spp. } \\
\text { Bulimina spp. } \\
\text { Melonis barleeanus } \\
\text { Melonis pompilioides } \\
\text { Oridorsalis umbonatus } \\
\text { Cassidulina teretis }\end{array}$ & \multirow[t]{5}{*}{$\begin{array}{l}\text { Bulimina spp. } \\
\text { Cibicides } \mathrm{cf} . \\
\quad \text { C. refuigens } \\
\text { Melonis zaandamae } \\
\text { (=M. barleeanus?) } \\
\text { Islandiella teretis } \\
\text { (=Cassidulina teretis) }\end{array}$} \\
\hline Pliocene & early & $\begin{array}{l}\text { Epistominella exigua } \\
\text { Globocassidulina } \\
\quad \text { subglobosa } \\
\text { Gyroidinoides spp. } \\
\text { Nodosaria/Stilostomella spp. } \\
\text { Oridorsalis umbonatus } \\
\text { Planulina wuellerstorfi } \\
\text { Cibicidoides kullenbergi } \\
\text { Uvigerina compressa }\end{array}$ & $\begin{array}{l}\text { Sigmoilopsis schlumbergeri } \\
\text { Pyrgo lucernula } \\
\text { Pyrgo murrhina } \\
\text { Uvigerina spp. } \\
\text { Bulimina alazanensis } \\
\text { Bulimina striata } \\
\text { Cibicidoides spp. } \\
\text { Globocassidulina } \\
\quad \text { subglobosa }\end{array}$ & \\
\hline \multirow{3}{*}{ Miocene } & late & Bulimina alazanensis & $\begin{array}{l}\text { Gyroidinoides spp. } \\
\text { Oridorsalis umbonatus } \\
\text { Planulina wuellerstorfi }\end{array}$ & \\
\hline & middle & $\begin{array}{l}\text { Epistominella exigua } \\
\text { Globocassidulina subglobosa } \\
\text { Gyroidinoides spp. } \\
\text { Nodosaria/Stilostomella spp. } \\
\text { Oridorsalis umbonatus } \\
\text { Planulina wuellerstorfi } \\
\text { Cibicidoides kullenbergi }\end{array}$ & $\begin{array}{l}\text { Brizalina subaenariensis } \\
\text { Globocassidulina subglobosa } \\
\text { Gyroidinoides spp. } \\
\text { Nodosaria/Stilostomella spp. } \\
\text { Oridorsalis umbonatus } \\
\text { Planulina wuellerstorfi }\end{array}$ & \\
\hline & early & $\begin{array}{l}\text { Cibicidoides spp. } \\
\text { Cibicides sp. } \\
\text { Gyroidinoides spp. } \\
\text { Nodosaria/Stilostomella spp. } \\
\text { Siphonina tenuicarinata } \\
\text { Oridorsalis ecuadorensis } \\
\text { Oridorsalis umbonatus }\end{array}$ & $\begin{array}{l}\text { Cibicidoides spp. } \\
\text { Siphonina tenuicarinata } \\
\text { Planulina renzi } \\
\text { Gyroidina complanata } \\
\text { Anomalina alazanensis } \\
\text { Oridorsalis ecuadorensis } \\
\text { Globocassidulina subglobosa }\end{array}$ & \\
\hline
\end{tabular}

\section{APPENDIX A}

Faunal Reference List

\section{Neogene}

Astrononion guadelupae (Parker) = Melonis guadelupae Parker, 1964.

Bolivina cf. B. thalmanni Renz, 1948.

Brizalina subaenariensis (Cushman) = Bolivina subaenariensis Cushman, 1922.

Bulimina aculeata d'Orbigny, 1826.

Bulimina alazanensis Cushman, 1927.

Bulimina striata d'Orbigny, 1843.

Cassidulina obtusa Williamson, 1858.

Cassidulina teretis Tappan, 1951.

Cibicidoides bradyi (Trauth) = Truncatulina bradyi Trauth, 1942.

Cibicidoides kullenbergi (Parker) = Cibicides kullenbergi Parker, 1953.

Cibicidoides robertsonianus (Brady) = Truncatulina robertsoniana Brady, 1881.

Eggerella bradyi $($ Cushman $)=$ Verneuilina bradyi Cushman, 1911. Ehrenbergina serrata Reuss, 1850.

Ehrenbergina trigona Goës = Ehrenbergina serrata Reuss var. trigona Goës, 1896.

Epistominella exigua $($ Brady) $=$ Pulvinulina exigua Brady, 1886. Eponides tumidulus (Brady) = Truncatulina tumidula, Brady, 1884 . Francesita advena $($ Cushman $)=$ Virgulina advena Cushman, 1922.

Fursenkoina schreibersiana $($ Czjek) $=$ Virgulina schreibersiana $\mathrm{Cz}$ jzek, 1848.

Globocassidulina subglobosa $($ Brady) $=$ Cassindulina subglobosa Brady, 1881.

Hoeglundina elegans (d'Orbigny) = Rotalia elegans d'Orbigny, 1826. Karreriella bradyi (Cushman) = Gaudryina bradyi Cushman, 1911 .

Laticarinina pauperata $($ Parker and Jones) $=$ Pulvinulina repanda var. menardii subvar. pauperata Parker and Jones, 1865.
Melonis barleeanus (Williamson) $=$ Nonionina barleeana Williamson, 1858.

Melonis pompilioides (Fichtel and Moll) $=$ Nautilus pompilioides Fichtel and Moll, 1798.

Oridorsalis umbonatus (Reuss) = Rotalina umbonatus Reuss, 1851.

Osangularia culter (Parker and Jones) = Planorbulina culter Parker and Jones, 1865.

Osangularia rugosa $($ Phleger and Parker $)=$ Pseudo parrella(?) rugosa, Phleger and Parker, 1951.

Planulina wuellerstorfi (Schwager) = Anomalina wüllerstorfi Schwager, 1866.

Pullenia bulloides (d'Orbigny) $=$ Nonionina bulloides d'Orbigny, 1846 .

Pullenia osloensis Feyling-Hanssen, 1954 = Pullenia quinqueloba (Reuss) subsp. minuta Feyling-Hanssen, 1954.

Pullenia quinqueloba (Reuss) = Nonionina quinqueloba Reuss, 1851. Pullenia salisburyi Stewart and Stewart, 1930.

Pyrgo bulloides d'Orbigny, 1826.

Pyrgo murrhina (Schwager) = Biloculina murrhina Schwager, 1886.

Rectuvigerina royoi Bermudez and Fuenmayor, 1963.

Sigmoilopsis schlumbergeri (Silvestri) = Sigmoilina schlumbergeri Silvestri, 1904

Siphonina tenuicarinata Cushman, 1927.

Siphotextularia catenata $($ Cushman) $=$ Textularia catenata Cushman, 1911.

Sphaeroidina bulloides d'Orbigny, 1826

Spiroloculina pusilla Earland, 1935.

Spiroplectammina spectabilis (Gryzbowski) $=$ Spiroplecta spectabilis Gryzbowski, 1898.

Triloculina frigida Lagoe, 1977.

Uvigerina auberiana d'Orbigny, 1839.

Uvigerina compressa Cushman, 1926.

Uvigerina peregrina Cushman, 1923. 
Vulvulina pennatula (Batsch) $=$ Nautilus $($ Orthoceras) pennatula Batsch, 1791.

Because of the taxonomic confusion surrounding the species of Gyroidinoides, no attempt has been made to identify them. Likewise, specimens of Nodosaria, Stilostomella and other uniserial genera are often broken and do not show the detail necessary for specific or generic assignment.

\section{Paleogene}

(Species already listed under Neogene are omitted)

Alabamina obtusa $($ Burrows and Holland $)=$ Pulvinulina exigua $(\mathrm{Bra}-$ dy) var. obtusa Burrows and Holland, 1897.

Alabamina wilcoxensis Toulmin, 1940.

Anomalinoides acutus (Plummer) = Anomalina ammonoides (Reuss) var. acuta Plummer, 1926.

Anomalinoides howelli $($ Toulmin $)=$ Cibicides howelli Toulmin, 1941 . Anomalinoides nobilis Brotzen, 1948.

Bolivinopsis adamsi (Lalicker) = Spiroplectammina adamsi Lalicker, 1935.

Bulimina praeinflata Bykova, 1958.

Bulimina trigonalis Ten Dam, 1944.

Bulimina tuxpamensis Cole, 1928.

Cancris subconicus (Terquem) $=$ Rotalina subconica Terquem, 1882.

Cibicides west $i$ Howe, 1939.

Cibicides alleni (Plummer) = Truncatulina alleni Plummer, 1927.

Cibicidoides succedens Brotzen, 1948.

Elphidium hiltermanni Hagn, 1952

Epistominella vitrea, Parker, 1953.

Eponides umbonatus (Reuss) $=$ Rotalina umbonata Reuss, 1851.

Florilus elongatum (d'Orbigny) = Nonionina elongata d'Orbigny, 1826.

Gaudryina hiltermanni $($ Meisl) $=$ Gaudryina $($ Pseudogaudryina $)$ hiltermanni Meisl, 1959.

Gavelinella semicribrata $($ Beckmann) $=$ Anomalina pompilioides Galloway \& Heminway var. semicribrata, Beckman, 1953.

Glandulina laevigata (d'Orbigny) $=$ Nodosaria (Glandulina) laevigata d'Orbigny, 1826

Loxostomoides applinae (Plummer) = Bolivina applinae Plummer, 1927.

Nonion havanensis Cushman and Bermudez, 1937.

Nonion laeve (d'Orbigny) = Nonionina laevis d'Orbigny, 1826 .

Nuttallides truempyi (Nuttall) $=$ Eponides trümpyi, Nuttall, 1930.

Oridorsalis ecuadorensis (Galloway and Morray) = Rotalia ecuadorensis Galloway and Morray. 1929.
Osangularia expansa (Toulmin) $=$ Parrella expansa Toulmin, 1941.

Pararotalia calvezae Loeblich and Tappan, 1957.

Pararotalia curryi Loeblich and Tappan, 1957.

Praeglobobulimina ovata (d'Orbigny) = Bulimina ovata d'Orbigny, 1846.

Pulsiphonina prima $($ Plummer) $=$ Siphonina prima Plummer, 1927. Siphonina lamarckana, Cushman, 1927.

Trifarina cuneata (Brotzen) = Angulogerina cuneata Brotzen, 1948.

Turrilina brevispira Ten Dam, 1944.

Uvigerina abbreviata Terquem, 1882.

\section{APPENDIX B \\ List of Macrofossils}

Macrofossils were encountered at a number of levels in the Paleogene. Normally they were evident only because they were sawn through when the cores were split. Therefore, few are complete. No attempt has been made to identify them in detail because their incomplete and often fragmentary nature would make this very difficult.

Sample 553A-14-1, 155-146 cm: fragment of thin, smooth-shelled, bivalved mollusk. Early Eocene. NP10.

Sample 553A-15-3, $132 \mathrm{~cm}$ : fragments of Turritella. Early Eocene. NP10. Sample 553A-15-3, $134 \mathrm{~cm}$ : irregular echinoid. Early Eocene. NP10.

Sample 553A-10-6, $55 \mathrm{~cm}$ : smooth, thin-shelled bivalved mollusk. Early Eocene. NP10.

Sample 553A-28-1, $15 \mathrm{~cm}$ : serpulid worm tubes. Early Eocene. ?NP10.

Sample 553A-36-1, $90 \mathrm{~cm}$ : smooth, thin-shelled, infaunal bivalve; both valves preserved. Late Paleocene. ?NP9.

Sample 553A-37-3, $95 \mathrm{~cm}$ : thick-shelled oyster. Late Paleocene. ?NP9.

Sample 554A-6-2, 73-77 cm: dasyclad alga, Dactylopora. Early Eocene. NP11.

Sample 555-28-2, bottom: Nucula. Early Eocene. NP11.

Sample 555-33-2, $88 \mathrm{~cm}$ : thin-shelled oyster. Early Eocene. NP10.

Sample 555-33-2, $105 \mathrm{~cm}$ : oyster. Early Eocene. NP10.

Sample 555-33-2, $110 \mathrm{~cm}$ : smooth, thick-shelled bivalved mollusk. Both valves preserved together. Early Eocene. NP10.

Sample 555-38-2, 73-75 cm: fragments of ?actaeonid gastropod. Early Eocene, NP10.

Sample 555-40,CC Nucula. Early Eocene. NP10.

Sample $555-41-4,15 \mathrm{~cm}$ : solitary coral. Early Eocene, NP10.

Sample 555-46,CC. tiny, smooth bivalved molluscs. ?Late Paleocene. ?NP9.

Sample 555-49,CC: thick-shelled oysters. ?Late Paleocene. ?NP9.

Sample 555-58-2, $77 \mathrm{~cm}$ : solitary coral. ?Late Paleocene. ?NP9.

Sample 555-59-4, $136 \mathrm{~cm}$ : solitary coral plus actaeonid gastropod. ?Late Paleocene. ?NP9. 


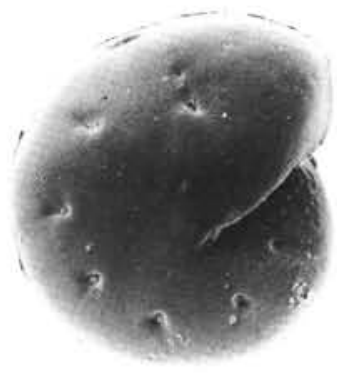

1

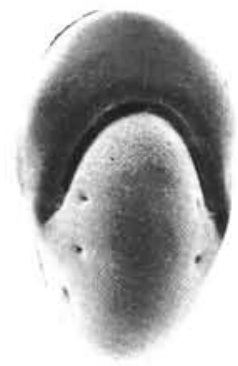

2

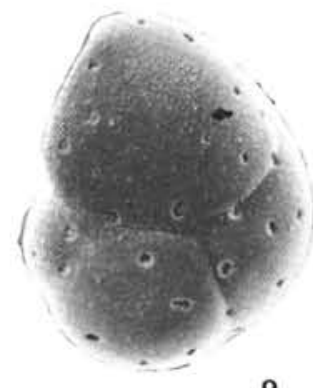

9

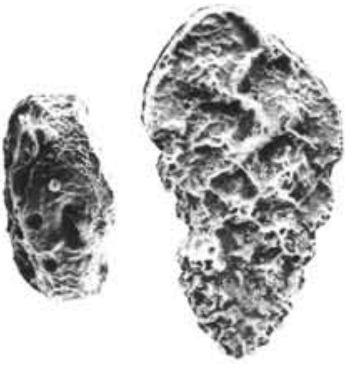

3

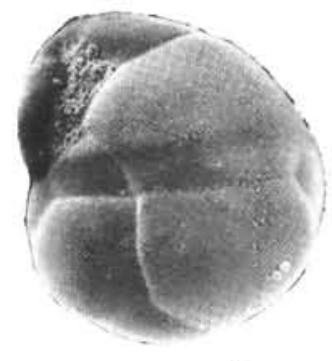

10
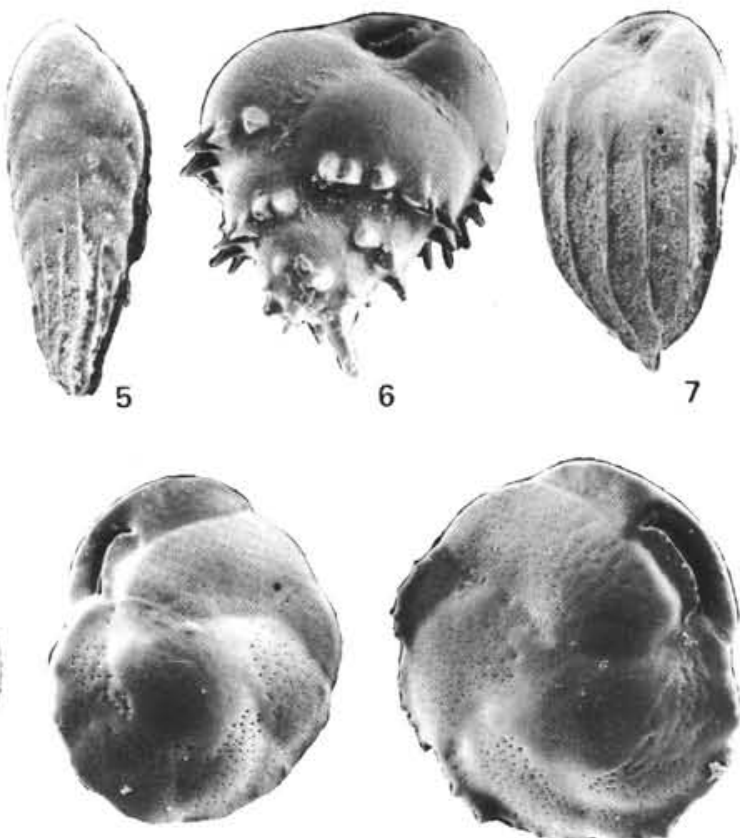

11

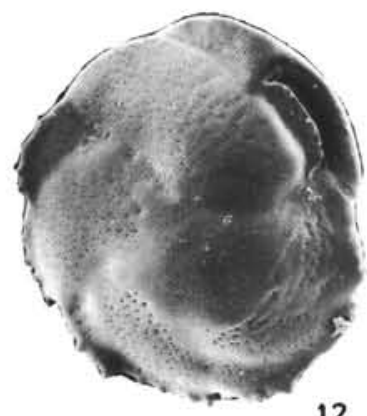

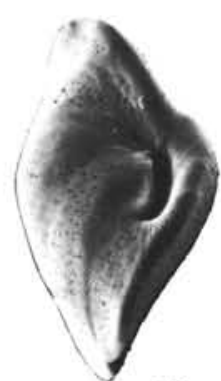

13

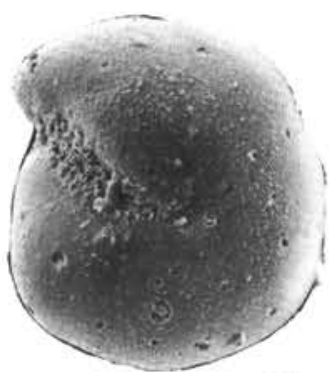

14

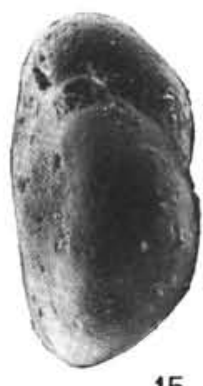

15

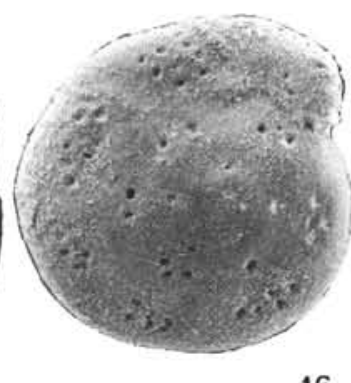

16

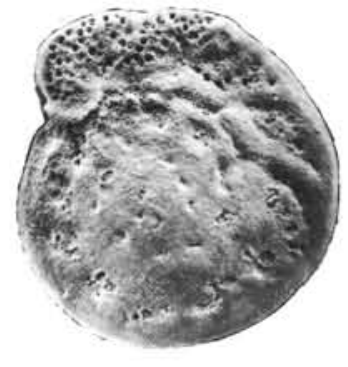

17

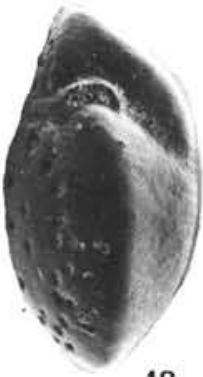

18

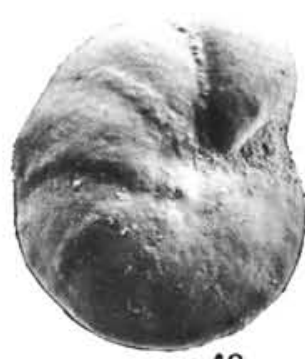

19

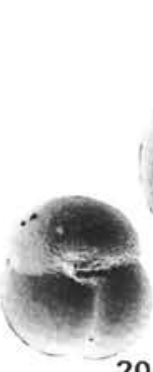

20

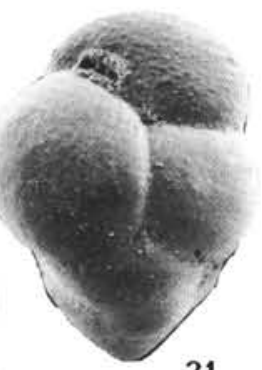

21

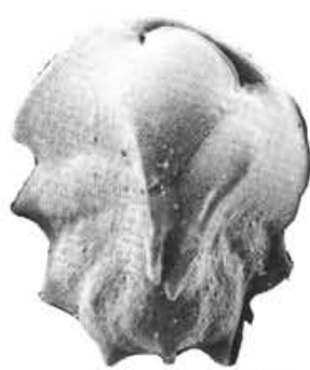

22

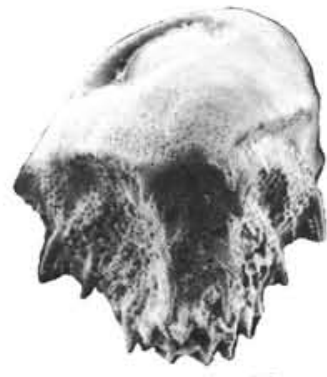

23

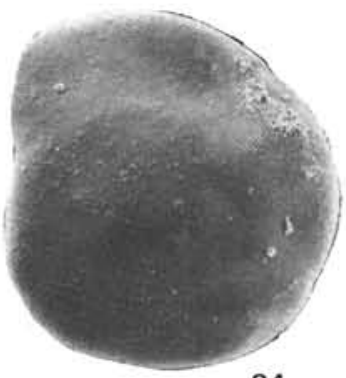

24

Plate 1. Neogene foraminifers. 1, 2. Astrononion guadelupe (Parker), $\times 150$; Sample 554A-2-2, $3 \mathrm{~cm}$; late Miocene. 3, 4. Bolivina cf. B. thalmanni Renz, $\times 105$; Sample 552A-1,CC; Pleistocene. 5. Brizalina subaenariensis (Cushman), $\times 80$; Sample 552A-16,CC; early Pliocene. 6. Bulimina aculeata d'Orbigny, $\times 165$; Sample 551A-1,CC; Pleistocene. 7. Bulimina alazanensis Cushman, $\times 80$; Sample 552A-16,CC; early Pliocene. 8. Bulimina striata d'Orbigny, $\times 100$; Sample 552A-34,CC; late Miocene. 9, 10. Cassidulina obtusa Williamson, $(9) \times 200$, (10) $\times 175$; Sample 552A-22,CC; early Pliocene. 11, 12, 13.Cassidulina teretis Tappan, $(11) \times 100,(12) \times 85,(13) \times 150$; Sample 552 A-3,CC; Pleistocene. 14, 15, 16. Cibicidoides bradyi (Trauth), $(14) \times 175,(15) \times 140,(16) \times 110$; Sample $555-11$, CC; late Miocene. 17, 18, 19. Cibicidoides kullenbergi (Parker), $(17,18) \times 65,(19) \times 95$; Sample 555-5,CC; late Miocene. 20,21 . Eggerella bradyi $($ Cushman), (20) $\times 56,(21) \times 87$; Sample 552A-14,CC; Pleistocene. 22. Ehrenbergina serrata Reuss, $\times 100$; Sample 552A-14,CC; early Pliocene. 23. Ehrenbergina trigona Goës, $\times 100$; Sample 552A-10,CC; late Pliocene. 24. Epistominella exigua (Brady), $\times 175$; Sample 552A-4,CC; Pleistocene. 


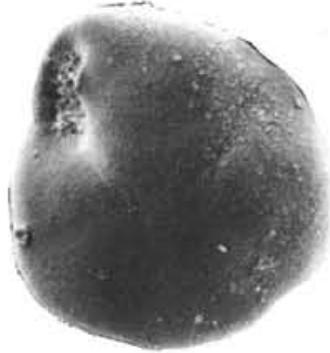

1

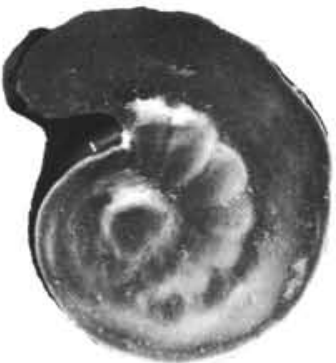

7
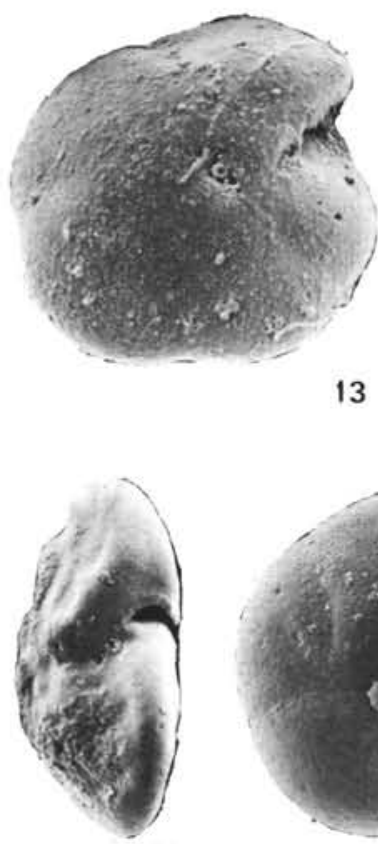

18

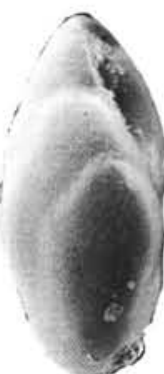

2

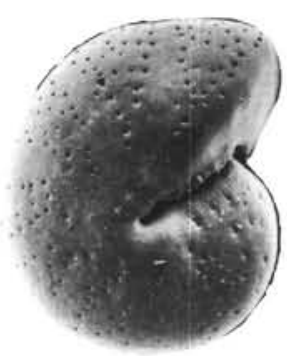

8

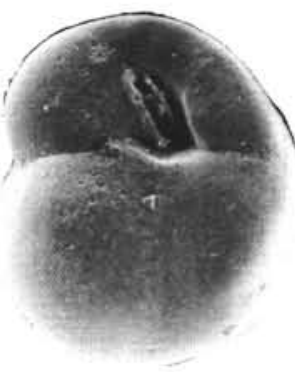

3

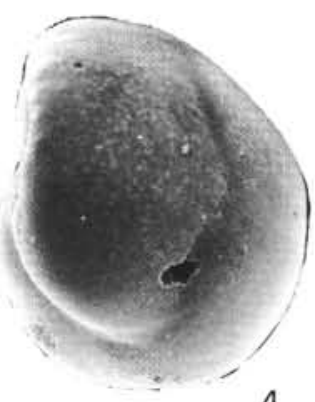

4

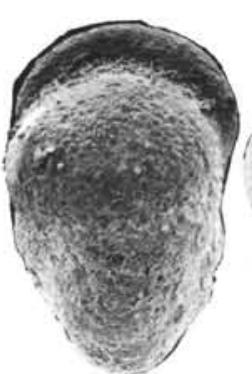

5

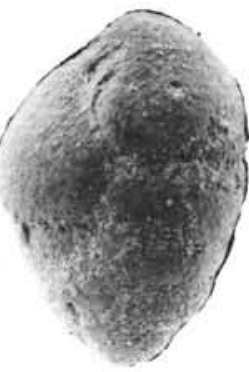

6

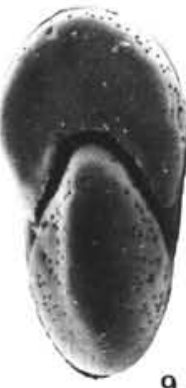

9

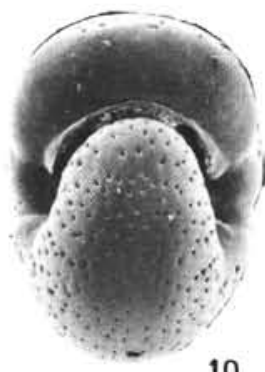

10

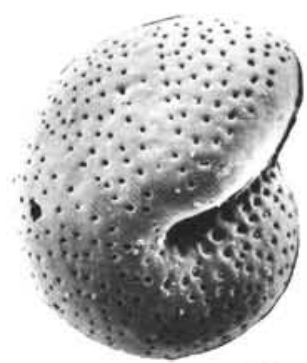

11

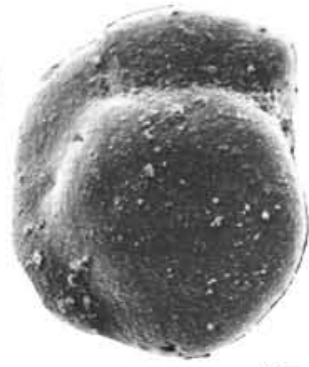

12
13

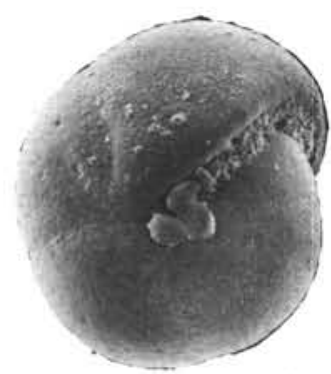

19

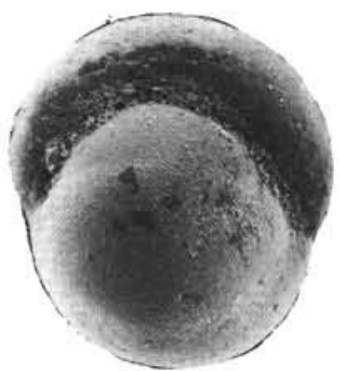

20

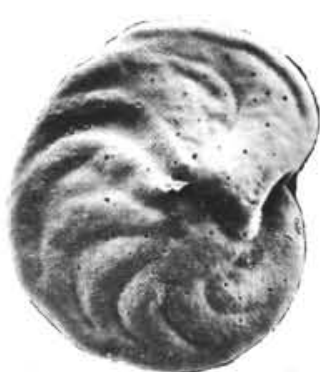

16

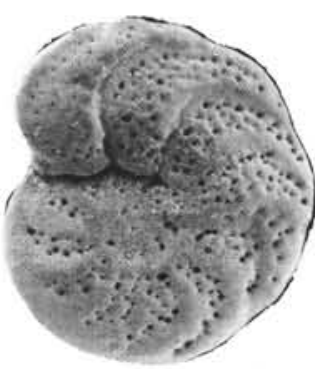

17

Plate 2. Neogene foraminifers. 1, 2. Epistominella exigua (Brady), (1) $\times 175,(2) \times 165$; Sample 552A-4,CC; Pleistocene. 3, 4. Globocassidulina subglobosa (Brady) $\times 195$; Sample 552A-34,CC; late Miocene. 5, 6. Karreriella bradyi (Cushman), $(5) \times 100$, (6) $\times 80$; Sample $555-17$, CC; late Miocene. 7. Laticarinina pauperata (Parker and Jones), $\times 40$; Sample 552A-16,CC; early Pliocene. 8, 9. Melonis barleeanus (Williamson), $(8) \times 140,(9) \times 120$; Sample 552A-1,CC; Pleistocene. 10, 11. Melonis pompilioides (Fichtel and Moll), $(10) \times 85,(11) \times 120$; Sample 552A-3,CC; Pleistocene. 12, 13. Oridorsalis umbonatus (Reuss), $\times 175$; Sample 555-14,CC; late Miocene. 14, 15. Osangularia rugosa (Phleger and Parker), (14) $\times 165$, (15) $\times 185$; Sample 555-24-7, $55 \mathrm{~cm}$; early Miocene. 16, 17, 18. Planulina wuellerstorfi (Schwager), (16) $\times 70$, (17) $\times 60,(18) \times 90 ; 16,18$ : Sample 552A-1,CC, Pleistocene, 17: Sample 552A-34,CC, late Miocene. 19, 20. Pullenia bulloides (d'Orbigny), 130; Sample 555-15,CC; late Miocene. 21, 22. Pullenia osloensis Feyling-Hanssen, X195; Sample 552A-34,CC; late Miocene. 


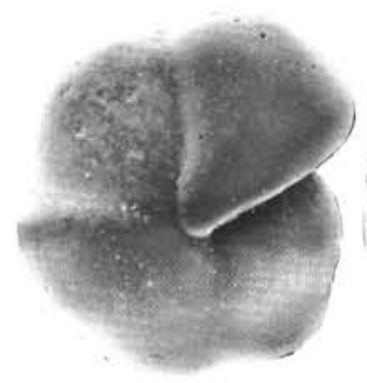

1

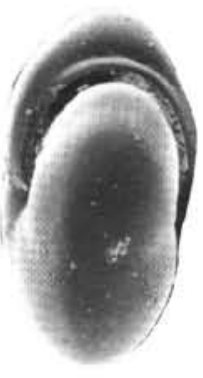

2

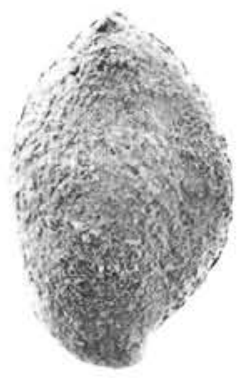

3

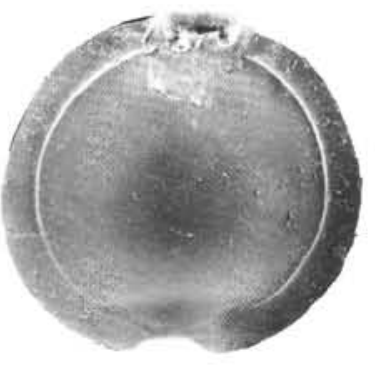

4

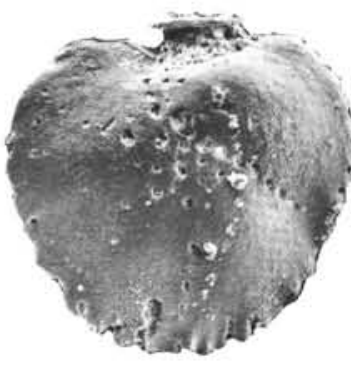

5

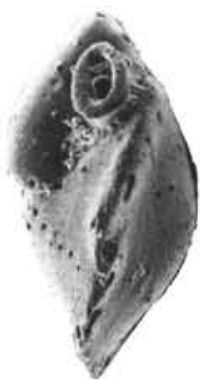

6
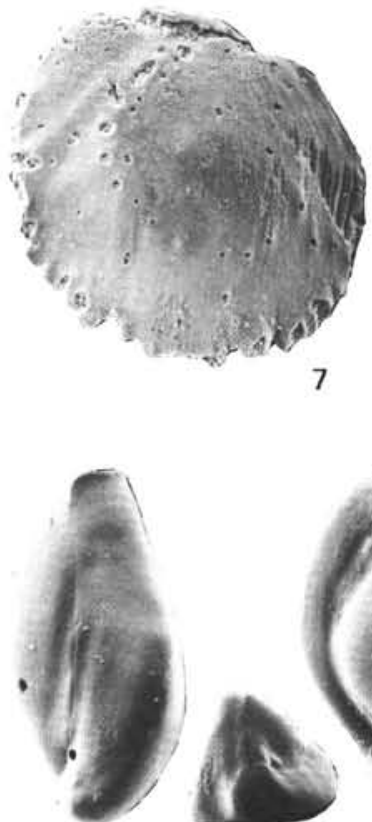

12

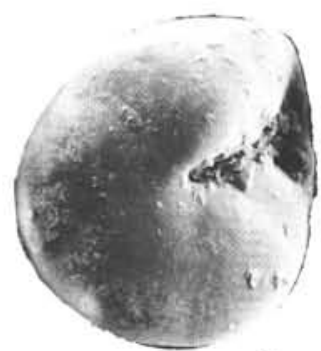

19

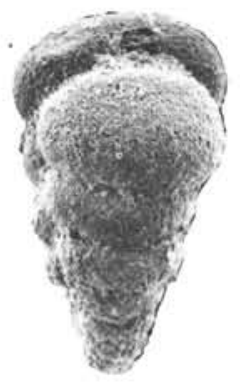

8

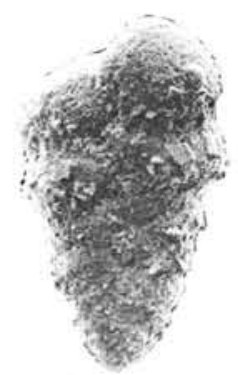

9

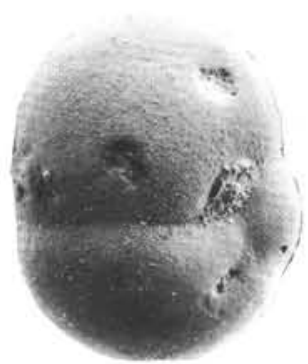

10

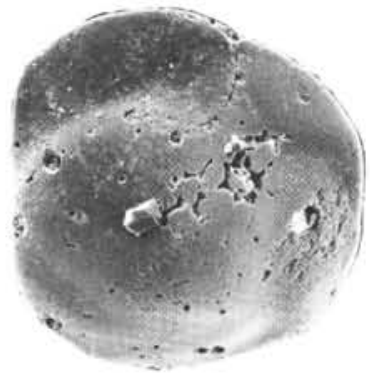

17

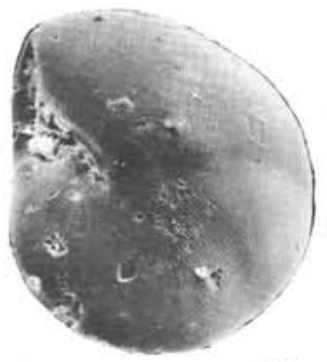

23

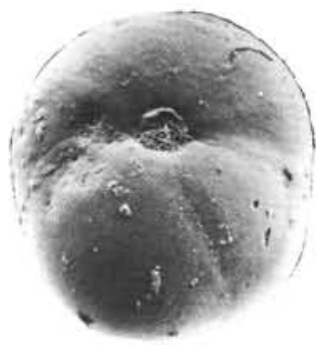

11

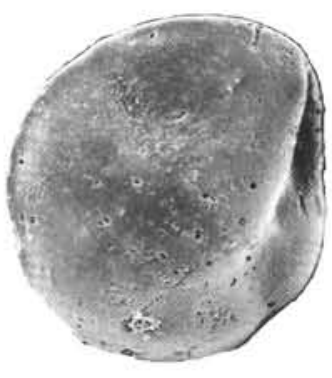

18

Plate 3. Neogene and Paleogene foraminifers. 1, 2. Pullenia quinqueloba (Reuss) $\times 140$, Sample 552A-5,CC; Pleistocene. 3. Sigmoilopsis schlumbergeri (Silvestri) $\times$ 40; Sample 555-mudline; ?Recent. 4. Pyrgo murrhina (Schwager), ×33; Sample 555-mudline; ?Recent. 5 , 6, 7. Siphonina tenuicarinata Cushman, $\times 140$; Sample 555-22,CC: mid-Miocene. 8, 9. Siphotextularia catenata $($ Cushman), $(8) \times 130,(9) \times 85$; Sample 553A-2,CC; late Pliocene. 10,11. Sphaeroidina bulloides d'Orbigny, $\times 140$; Sample 555-5,CC; late Miocene. 12, 13, 14. Triloculina frigida Lagoe, (12) $\times 140$, (13) $\times 120$, (14) $\times 155$; Sample 552A-1,CC; Pleistocene. 15. Uvigerina auberiana d'Orbigny, $\times 130$; Sample 5555,CC; late Miocene. 16. Uvigerina compressa Cushman, $\times 100$; Sample 552A-34,CC; late Miocene. 17, 18. Alabamina $\mathrm{sp}$., $\times 140$; Sample 553A-9-1, $99 \mathrm{~cm}$; early Miocene. 19, 20, 21. Alabamina obtusa (Burrows and Holland), $\times 155$; Sample 555-47,CC; ?late Paleocene. 22, 23, 24. Alabamina wilcoxensis Toulmin, $\times 155$; Sample 553A-10-3, $66 \mathrm{~cm}$; mid-Eocene. 


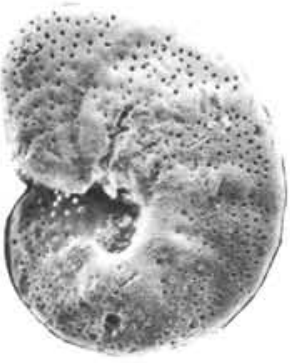

1

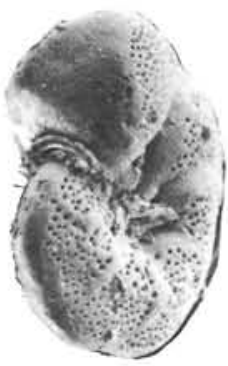

2

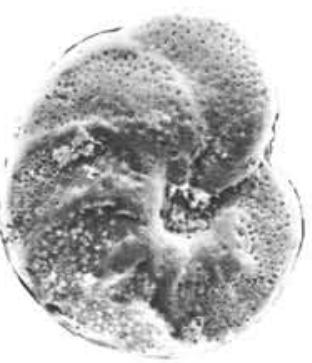

3

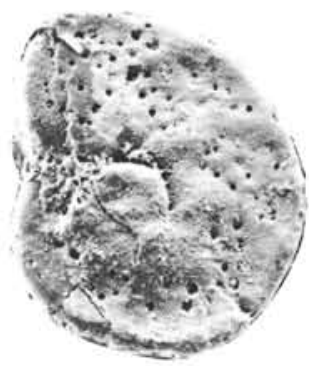

4

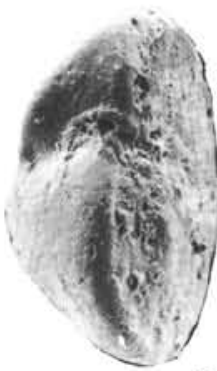

5

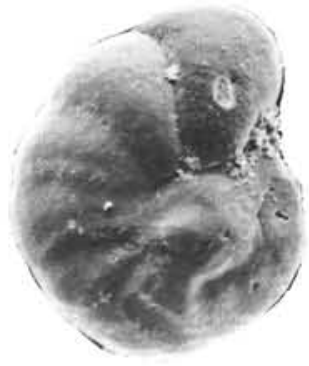

6

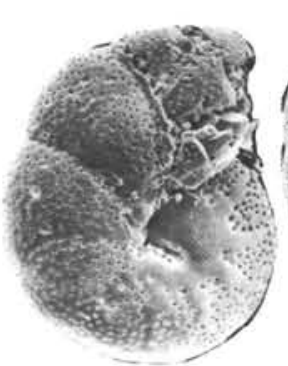

7

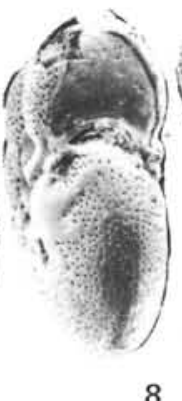

8

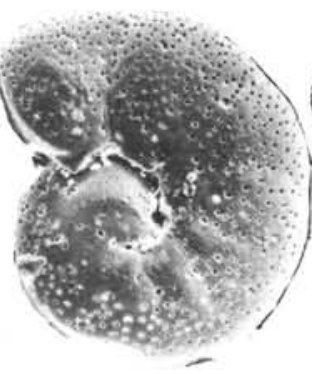

9

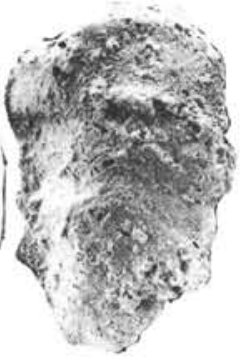

10

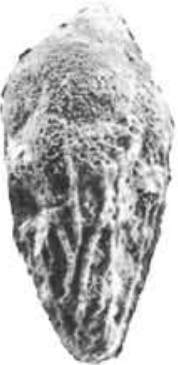

11

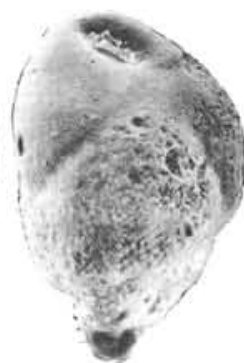

12

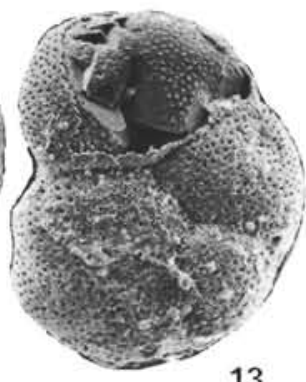

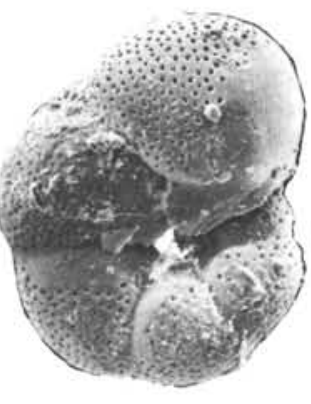

14

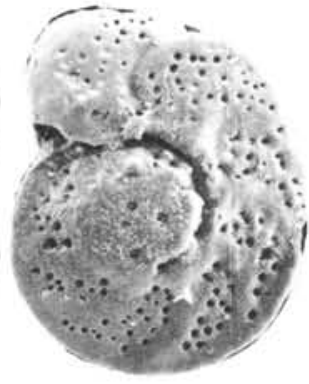

15

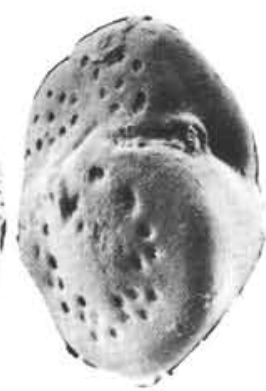

16

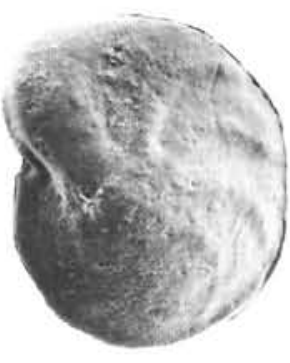

17

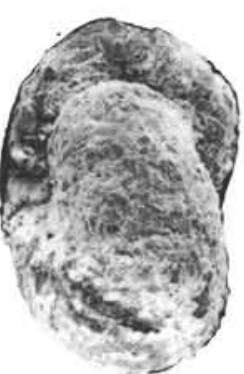

18

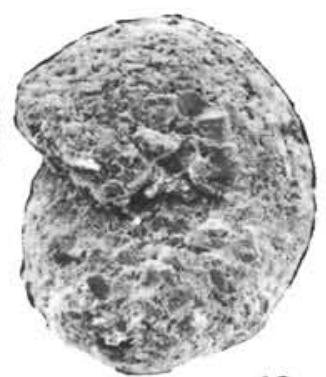

19

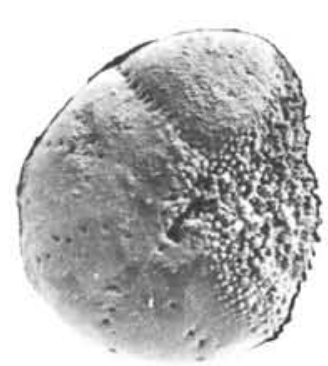

20

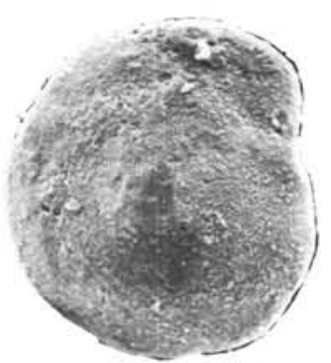

21

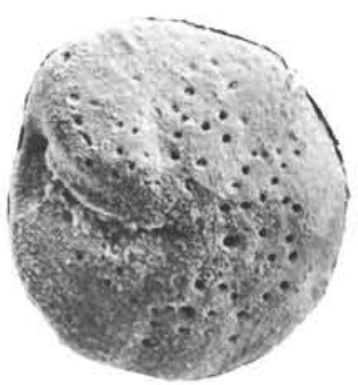

22

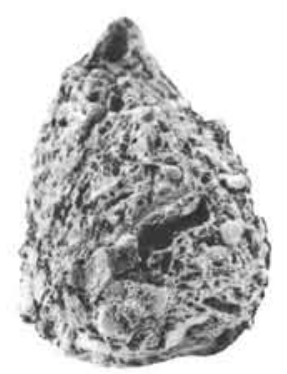

23

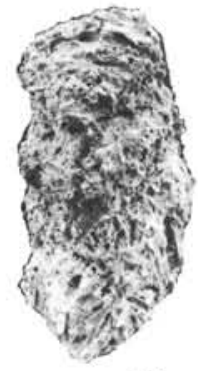

24

Plate 4. Paleogene foraminifers. 1, 2, 3. Anomalinoides acutus (Plummer), (1) $\times 130,(2,3) \times 110$; Sample 553A-22,CC; early Eocene. 4, 5, 6. Anomalinoides howelli (Toulmin), $(4,5) \times 185,(6) \times 165$; Sample 552-12, CC; early Eocene. 7, 8, 9. Anomalinoides nobilis Brotzen (7, 9) $\times 120$; Sample 555-41,CC; early Eocene. 10. Bolivinopsis adamsi (Lalicker), $\times 70$; Sample 554A-12-1, $35 \mathrm{~cm}$; early Eocene. 11. Bulimina trigonalis Ten Dam, $\times 165$; Sample 555-33,CC; early Eocene. 12. Bulimina tuxpamensis Cole, $\times 155$; Sample 552A-37-3, $67 \mathrm{~cm}$; mid-Eocene. 13, 14. Cancris subconicus (Terquem) $\times 140$; Sample 553A-37-2, $28 \mathrm{~cm}$; late Paleocene. 15, 16, 17. Cibicidoides alleni (Plummer), (15, 17) $\times 110,(16) \times 155$; Sample 553A-24,CC. ?late Paleocene. 18, 19. Cribrostomoides sp., $\times 55$; Sample 553A-22,CC; early Eocene. 20. Elphidium hiltermanni Hagn, $\times 155$; Sample 553A-37-2, 28 cm; late Paleocene. 21, 22. Epistominella vitrea Parker, $\times 370$; Sample 553A-23,CC: early Eocene. 23, 24. Gaudryina hitermanni (Meisl), $(23) \times 200,(24) \times 120$; Sample 553A-37-2, $28 \mathrm{~cm}$; late Paleocene. 


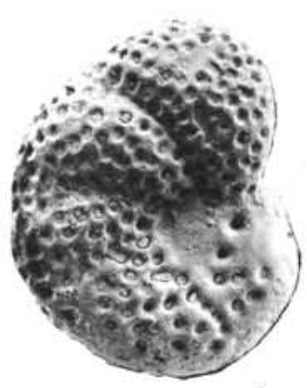

1

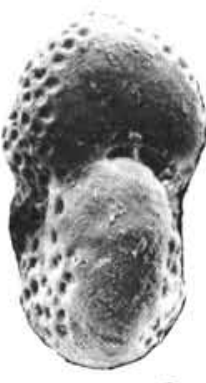

2

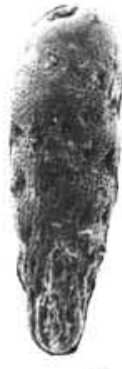

3

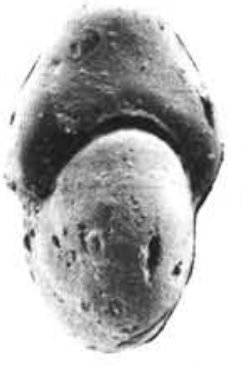

4

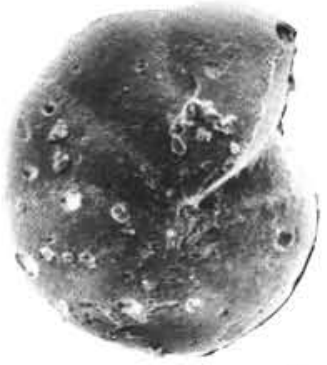

5

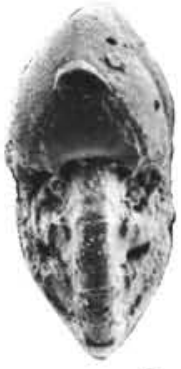

6

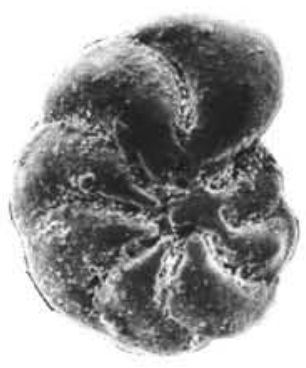

7

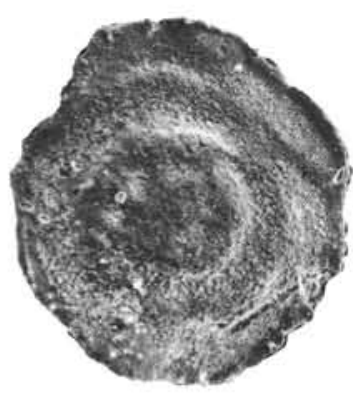

8

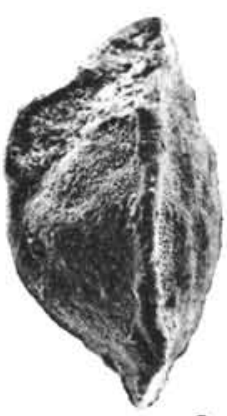

9

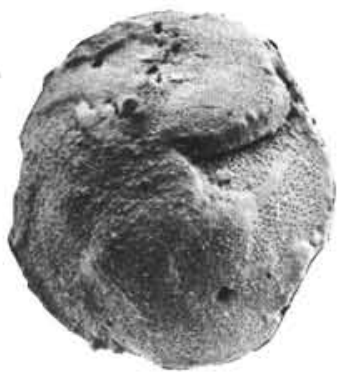

10

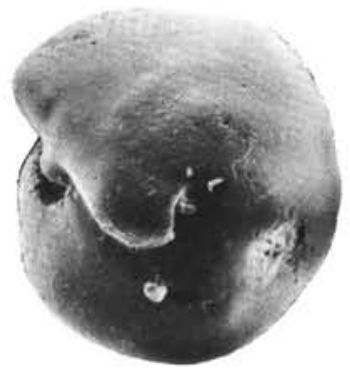

11

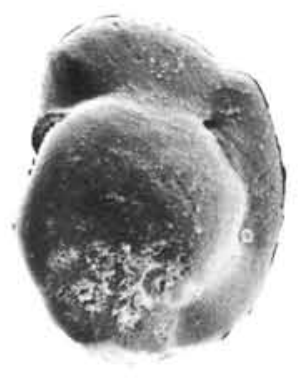

12

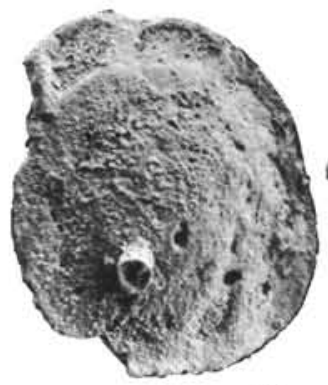

13

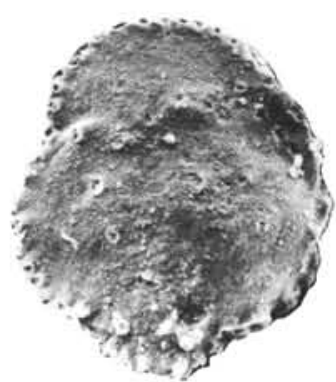

19

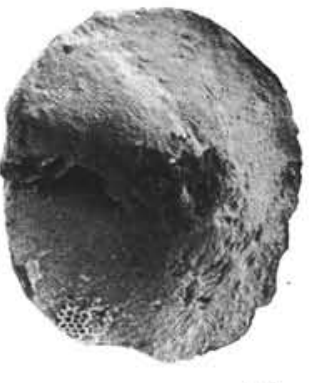

14

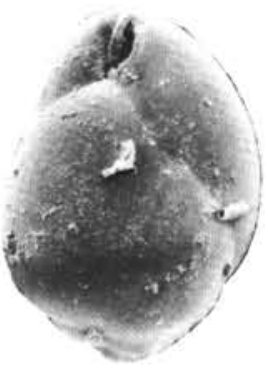

15

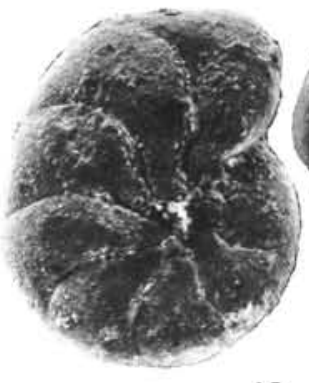

16

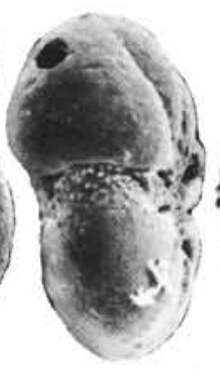

17

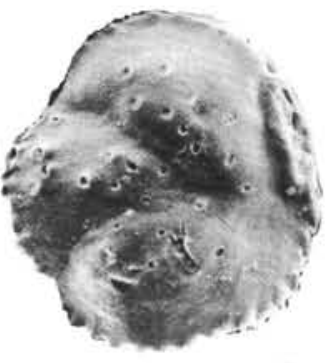

18

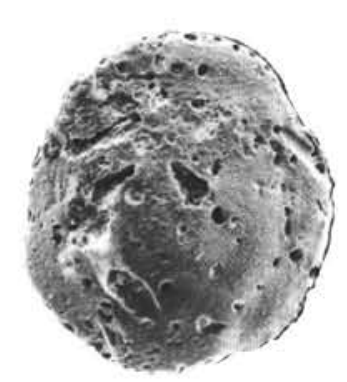

20

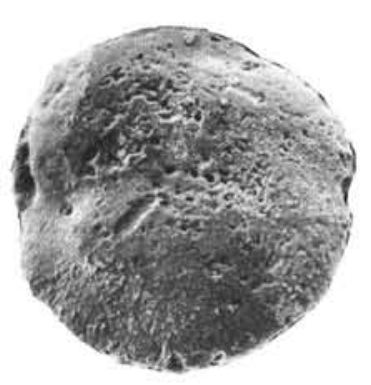

21

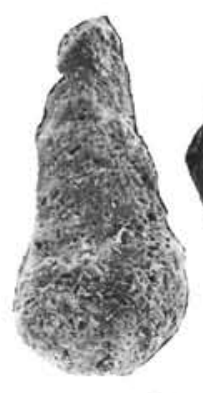

22

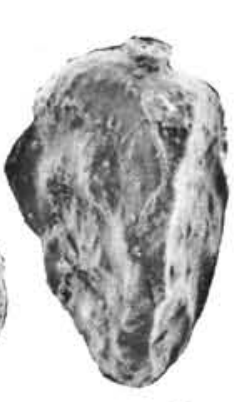

23

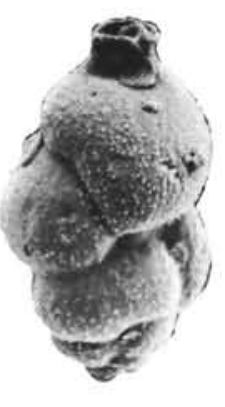

24

Plate 5. Paleogene foraminifers. 1, 2. Gavellinella semicribrata (Beckmann), $\times 80$; Sample 553A-25,CC; ?late Paleocene. 3. Loxostomoides applinae (Plummer) $\times 65$; Sample 555-61,CC; late Paleocene. 4, 5. Nonion havanensis Cushman and Bermudez, $\times 155$; Sample 553A-10-3, $135 \mathrm{~cm}$; mid-Eocene. 6, 7. Nonion laeve (d'Orbigny), $\times 155$; Sample 553A-37-2, $28 \mathrm{~cm}$; late Paleocene. 8, 9, 10. Nuttallides truempyi (Nuttall), $\times 100$; Sample 552A-37-1, $137 \mathrm{~cm}$ : mid-Eocene. 11, 12. Oridorsalis ecuadorensis (Galloway and Morrey), $(11) \times 200,(12) \times 165$; Sample 553A-9-6, $51 \mathrm{~cm}$; mid-Eocene. 13, 14. Osangularia expansa (Toulmin), (13) $\times 110$, (14) $\times 130$; Sample 552-21-2, $117 \mathrm{~cm}$; early Eocene. 15. Praeglobobulimina ovata (d'Orbigny), $\times 95$; Sample 553A-37-2, $28 \mathrm{~cm}$; late Paleocene. 16, 17. Protelphidium sp., $(16) \times 150$, (17) $\times 175$; Sample 555-52,CC; late Paleocene. 18, 19. Pulsiphonina prima (Plummer), $\times 190$; Sample 553A-23,CC; early Eocene. 20, 21. Siphonina lamarckana Cushman, $(20) \times 185,(21) \times 155$; Sample 553A-10,CC; early Eocene. 22. Spiroplectammina spectabilis (Gryzbowski), $\times 110$; Sample 554A-4-2, $47 \mathrm{~cm}$; late Oligocene. 23. Trifarina cuneata (Brotzen), $\times 120$; Sample 553A-24,CC; ?late Paleocene. 24. Uvigerina abbreviata Terquem, $\times 165$; Sample 555-58,CC; late Paleocene. 